\title{
C-Glycosidic Genistein Conjugates and Their Antiproliferative Activity
}

\author{
Aleksandra Rusin, ${ }^{1}$ Maciej Chrubasik, ${ }^{2}$ Katarzyna Papaj, \\ Grzegorz Grynkiewicz, ${ }^{3}$ and Wiesław Szeja ${ }^{2}$ \\ ${ }^{1}$ Center for Translational Research and Molecular Biology of Cancer, Maria Sklodowska-Curie Memorial Cancer Center and \\ Institute of Oncology, Wybrzeze AK 15, 44-100 Gliwice, Poland \\ ${ }^{2}$ Department of Organic Chemistry, Bioorganic Chemistry and Biotechnology, Silesian University of Technology, \\ Krzywoustego 4, 44-100 Gliwice, Poland \\ ${ }^{3}$ Pharmaceutical Research Institute, Rydygiera 8, 01-793 Warsaw, Poland
}

Correspondence should be addressed to Wiesław Szeja; wieslaw.szeja@adres.pl

Received 25 October 2012; Revised 22 March 2013; Accepted 2 April 2013

Academic Editor: Mehmet Emin Duru

Copyright ( 2013 Aleksandra Rusin et al. This is an open access article distributed under the Creative Commons Attribution License, which permits unrestricted use, distribution, and reproduction in any medium, provided the original work is properly cited.

\begin{abstract}
This paper presents our attempt to investigate scopes and the limitations of olefin cross-metathesis (CM) reaction in the synthesis of complex C-glycosides of genistein and evaluation of their antiproliferative activities. Novel genistein glycoconjugates were synthesized with the utility of CM reaction initiated by first and second generation of Grubbs catalysts. The relative reactivity of utilized olefins, based on categories proposed by Grubbs, was estimated. In vitro experiments in cancer cell lines showed that the selected derivatives (3a and $\mathbf{3 f}$ ) exhibited higher antiproliferative potential than the parent compound, genistein, and were able to block the cell cycle in the G2/M phase. The observed mechanism of action of C-glycosidic derivatives was similar to the activity of their O-glycosidic counterparts. These compounds were stable in culture medium. The obtained results show that our approach to genistein modification with application of cross-metathesis reaction allowed to obtain stable glycoconjugates with improved anticancer potential, compared to the parent isoflavone.
\end{abstract}

\section{Introduction}

The natural isoflavone, genistein abundant in soy-derived food and therefore a significant nonnutritional component of a diet is well known for at least two types of biological activity important for human health: hormonal (affinity to estrogen receptor beta) [1] and modulatory, influencing intracellular signaling and gene expression [2]. Epidemiological clinical and in vitro studies have shown that genistein might be a candidate for new drug in treatment of cardiovascular diseases, postmenopausal syndromes, and cancer [3-6]. Thus far, exploitation of these therapeutically prospective activities have not gone beyond early clinical trials, apparently because of rather poor drug-like properties of genistein (low lipophilicity combined with very low water solubility) reflected in pharmacokinetic parameters which practically exclude attaining of desired therapeutic concentrations of the active principle in the blood serum. It is postulated, however, that rational chemical derivatization of genistein can improve both pharmacokinetics and pharmacodynamics, including selectivity and efficacy of its biological action, and such claims have found experimental support in numerous studies [7]. Several groups have synthesized various derivatives of genistein with the intention to either improve biochemical and pharmacokinetic characteristics or to obtain compounds containing essential elements of the parent substance but having novel properties and/or affecting novel molecular targets [8-14]. In particular, research conducted by Polkowski and coworkers on O-glycosides of genistein should be mentioned $[15,16]$. In vitro studies have shown that those derivatives are able to inhibit proliferation of various cancer cell lines. In a follow-up study, it has been found that glycoconjugates linking genistein with an unsaturated pyranoside through an alkyl chain show antiproliferative activity against cancer 
cell lines, corroborating the working hypothesis, that an unsaturated sugar moiety is a carrier of certain structural features reflected in antiproliferative activity of genistein conjugates [17].

Pursuing further investigation of biological activity of genistein glycoconjugates, we decided to examine another type of constructs, less susceptible to biodegradation. Since O-glycosides can be easily hydrolyzed by enzymes present in the human body, we have focused on attachment of a sugar moiety via a C-glycosidic bond, reasoning that their greater stability will improve the bioavailability without affecting the antiproliferative activity. Although C-glycosylated flavonoids occur in nature, no practical synthetic alternative to the biogenetic pathway has been developed thus far. Therefore we decided to create a linkage between a carbohydrate moiety and a genistein molecule by an olefin cross-metathesis (CM) reaction with the easily accessible appropriate terminal olefins, that is, C-allyl glycosides and 7-O-allylgenistein.

Herein we present the synthesis of novel genistein glycoconjugates by application of olefin CM reaction initiated by first and second generation of Grubbs catalysts, their biological activity against selected tumor cell lines and stability in culture media.

\section{Experimental}

2.1. General Methods of Synthesis. Reactions were monitored by TLC, which was carried out on $0.25 \mathrm{~mm}$ Silica Gel F254 plates (Acros) using either UV light, a 5\% EtOH solution of $\mathrm{H}_{2} \mathrm{SO}_{4}$ with heat as developing agent or a chamber with silica gel saturated with $\mathrm{I}_{2}$. Acros Silica gel was used for column chromatography. ${ }^{1} \mathrm{H}$ and ${ }^{13} \mathrm{C}-\mathrm{NMR}$ spectra were recorded on a VARIAN INOVA $300(300 \mathrm{MHz}$ and $75 \mathrm{MHz}$, resp.) and on VARIAN 600 instrument (600 MHz and $150 \mathrm{MHz}$, resp.) using $\mathrm{Me}_{4} \mathrm{Si}$ as an internal reference.

2.2. General Procedure for Preparation of Grubbs Catalyst in Paraffin. Grubbs complex and paraffin were placed in a vessel sealed with septa. Then, air was removed by reduced pressure, and Ar gas was introduced. The vessel was placed in an ultrasonic $\mathrm{H}_{2} \mathrm{O}$ bath and warmed to about $60^{\circ} \mathrm{C}$ which resulted in melting of the paraffin and homogenization of catalyst in it. After cooling the mixture, a brown wax was obtained, which was stored at r.t. without inert gas protection. The catalyst prepared in that way was still active after a 6month period. The average concentration of Grubbs complex in paraffin was about $0.09 \mathrm{mmol} / \mathrm{g}$.

2.3. General Procedure for Olefin Cross-Metathesis Reaction. 7-O-Allylgenistein (2) and C-allylglycoside (1a-1h) were dissolved in $\mathrm{CH}_{2} \mathrm{Cl}_{2}$ (solubility of 2 is about $20 \mathrm{mg}$ per $1 \mathrm{~mL}$ ). Grubb's catalyst in paraffin was placed inside a reflux condenser and then the condenser, was attached to the reaction vessel. Air was removed under reduced pressure (mixture was allowed to boil for about $2 \mathrm{~min}$ ), and Ar gas was introduced. The mixture was heated at refluxed on a $\mathrm{H}_{2} \mathrm{O}$ bath. The reaction started when the catalyst was washed from the condenser by the refluxing solvent. After $3 \mathrm{~h}$, homodimer was removed from reaction mixture by filtration through a cotton wool, and the solvent was removed from the filtrate under reduced pressure. The residue was dissolved in toluene and chromatographed on silica-gel column.

2.4. Spectroscopic Data of Products Obtained in Olefin CrossMetathesis Reactions. NMR samples of metathesis products contained both $\mathrm{E}$ and $\mathrm{Z}$ isomers. Analysis was done with the help of 2D NMR spectra: ${ }^{1} \mathrm{H}-{ }^{1} \mathrm{H}$ cosy and ${ }^{1} \mathrm{H}-{ }^{13} \mathrm{C}$ hetcor.

\subsection{1. $1^{\prime \prime}, 4^{\prime \prime}-$ Bis-(5, $4^{\prime}$-dihydroxyisoflavonyl-7-oxy)-but- $2^{\prime \prime}$ - en (5)}

E Isomer. ${ }^{1} \mathrm{H}$ NMR $\left(300 \mathrm{MHz}, \mathrm{D}_{6}\right.$-DMSO) $\delta$ ppm: 12.96 (HO5, s 2H) $9.64\left(\mathbf{H O}^{\prime} \mathbf{4}^{\prime}, \mathrm{s}, 2 \mathrm{H}\right), 8.40\left(\mathbf{H}-2\right.$; s; 2H) $7.36\left(\mathbf{H}-\mathbf{2}^{\prime}, \mathbf{H}-\right.$ $\mathbf{6}^{\prime}$; d; $\left.J_{11,15-12,14}=8.6 \mathrm{~Hz} ; 4 \mathrm{H}\right) 6.80\left(\mathbf{H}-3^{\prime}, \mathbf{H}-5^{\prime}\right.$; d; $J_{12,14-11,15}=$ $8.6 \mathrm{~Hz}$; 4H) $6.67\left(\mathbf{H - 8}\right.$; ; $\left.J_{5-7}=2.2 \mathrm{~Hz} ; 2 \mathrm{H}\right) 6.42\left(\mathbf{H}-6\right.$; d; $J_{7-5}=$ $2.2 \mathrm{~Hz} ; 2 \mathrm{H}) 6.10\left(\mathbf{H}-\mathbf{2}^{\prime \prime}, \mathbf{H}-3^{\prime \prime} ; \mathrm{m} ; 2 \mathrm{H}\right) 4.75\left(\mathbf{H}^{\prime \prime} \mathbf{1}^{\prime \prime}, \mathbf{H}-\mathbf{4}^{\prime \prime} ; \mathrm{m}\right.$; $4 \mathrm{H})$.

$Z$ Isomer. ${ }^{1} \mathrm{H}$ NMR (300 MHz, d $\mathrm{d}_{6}$-DMSO) $\delta$ ppm: 12.93 (H-5; s; 2H) $9.64\left(\mathbf{H}^{\prime} \mathbf{4}^{\prime}\right.$; s; 2H) $8.38\left(\mathbf{H}-2\right.$; s; 2H) $7.36\left(\mathbf{H}^{\prime} \mathbf{2}^{\prime}, \mathbf{H}^{\prime} \mathbf{6}^{\prime}\right.$; $\left.\mathrm{d} ; J_{11,15-12,14}=8.6 \mathrm{~Hz} ; 4 \mathrm{H}\right) 6.80\left(\mathbf{H}-3^{\prime}, \mathbf{H}-5^{\prime}\right.$; d; $J_{12,14-11,15}=$ $8.6 \mathrm{~Hz}$; 4H) 6.69 (H-8; d; $\left.J_{5-7}=2.2 \mathrm{~Hz} ; 2 \mathrm{H}\right) 6.45\left(\mathbf{H}-6\right.$; d; $J_{7-5}=$ 2.2 Hz; 2H) 5.93 (H-2" $\mathbf{H}^{\prime \prime} \mathbf{H}-3^{\prime \prime}$; m; 2H) 4.87 (H-1" $^{\prime \prime}, \mathbf{H}-\mathbf{4}^{\prime \prime}$; m; $4 \mathrm{H})$.

Due to extremely low solubility of compound $\mathbf{5}$ we were unable to record ${ }^{13} \mathrm{C}$ NMR and HRMS spectra.

\subsection{2. $\quad 7^{\prime}-O-\left(1^{\prime \prime \prime}-C-(2,3,4,6-T e t r a-O-a c e t y l-\alpha-D-\right.$ glucopyrano- syl)-but-2 $\left.2^{\prime \prime \prime}-e n-4^{\prime \prime \prime}-y l\right)$-genistein ( $\left.3 a\right)$}

E Isomer. ${ }^{1} \mathrm{H}$ NMR (600 MHz, DMSO-D6) $\delta \mathrm{ppm:} 12.94$ $\left(\mathbf{O H}^{\prime}\right.$; s; 1H) $9.60\left(\mathbf{O H}^{\prime \prime}{ }^{\prime \prime}\right.$ s; $\left.1 \mathrm{H}\right) 8.39\left(\mathbf{H}-2^{\prime} ; \mathrm{s} ; 1 \mathrm{H}\right) 7.39$ $\left(\mathbf{H}^{\prime \prime}{ }^{\prime \prime}, \mathbf{H}-6^{\prime \prime}\right.$; d; $\left.J_{11^{\prime}-12^{\prime}, 15^{\prime}-14^{\prime}}=8.8 \mathrm{~Hz} ; 2 \mathrm{H}\right) 6.83\left(\mathbf{H}-3^{\prime \prime}, \mathbf{H}-\right.$

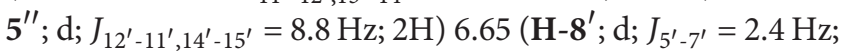
$1 \mathrm{H}) 6.40\left(\mathbf{H}-\mathbf{6}^{\prime}\right.$; d; $J_{7^{\prime}-5^{\prime}}=2.4 \mathrm{~Hz}$; 1H) $5.87\left(\mathbf{H}-3^{\prime \prime \prime}\right.$; dt; $J_{3^{\prime \prime}-2^{\prime \prime}}=$ $\left.14.6 \mathrm{~Hz} ; J_{3^{\prime \prime}-4^{\prime \prime}}=5.4 \times 2 \mathrm{~Hz} ; 1 \mathrm{H}\right) 5.81\left(\mathbf{H}-2^{\prime \prime \prime}\right.$; ddd; $J_{2^{\prime \prime}-3^{\prime \prime}}=$ $\left.14.6 \mathrm{~Hz} ; J_{2^{\prime \prime}-1 \mathrm{~L}^{\prime \prime}}=6.8 \mathrm{~Hz} ; J_{2^{\prime \prime}-1^{\prime \prime} \mathrm{a}}=6.3 \mathrm{~Hz} ; 1 \mathrm{H}\right) 5.30(\mathbf{H}-3 ; \mathrm{dd}$; $\left.J_{3-2}=8.8 \mathrm{~Hz} ; J_{3-4}=8.8 \mathrm{~Hz} ; 1 \mathrm{H}\right) 4.91\left(\mathbf{H}-2 ; \mathrm{dd} ; J_{2-3}=8.8 \mathrm{~Hz}\right.$; $\left.J_{2-1}=5.9 \mathrm{~Hz} ; 1 \mathrm{H}\right) 4.86\left(\mathbf{H}-4 ; \mathrm{dd} ; J_{4-3}=9.1 \mathrm{~Hz} ; J_{4-5}=9.1 \mathrm{~Hz} ; 1 \mathrm{H}\right)$ \left.4.64 (H-4" ; d; ${J^{\prime \prime}-3^{\prime \prime}}^{\prime \prime}=5.4 \mathrm{~Hz} ; 2 \mathrm{H}\right)$ 4.21-4.13 (H-1, H-6a; m; 2H) 4.00-3.93 (H-5, H-6b; m; 2H) $2.71\left(\mathbf{H}-\mathbf{1}^{\prime \prime \prime} \mathbf{a} ; \mathrm{m} ; J_{1^{\prime \prime} \mathrm{a}-1^{\prime \prime} \mathrm{b}}=\right.$ $\left.15.0 \mathrm{~Hz} ; J_{1^{\prime \prime} \mathrm{a}-2^{\prime \prime}}=10.9 \mathrm{~Hz} ; J_{1^{\prime \prime} \mathrm{a}-1}=7.6 \mathrm{~Hz} ; 1 \mathrm{H}\right) 2.32\left(\mathbf{H}_{-1} \mathbf{1}^{\prime \prime \prime} \mathbf{b} ; \mathrm{m}\right.$; 1H) $2.03 ; 2.00 ; 1.99 ; 1.98\left(\mathbf{C H}_{3}-; 12 \mathrm{H}\right)$.

${ }^{13} \mathrm{C}$ NMR $(150 \mathrm{MHz}, \mathrm{DMSO}-\mathrm{D} 6) \delta \mathrm{ppm}: 180.37\left(\mathbf{C}-\mathbf{4}^{\prime}\right)$ 170-169 (C=O × 4, C-6 $\left.{ }^{\prime}\right) 164.12\left(\mathbf{C}^{\prime} \mathbf{5}^{\prime}\right) 161.74\left(\mathbf{C}-4^{\prime \prime}\right) 157.49(\mathbf{C}-$ $\left.\mathbf{8 a}^{\prime}\right) 154.28\left(\mathbf{C}-2^{\prime}\right) 130.67\left(\mathbf{C}-2^{\prime \prime \prime}\right) 130.13\left(\mathbf{C}-2^{\prime \prime}, \mathbf{C}^{\prime \prime}{ }^{\prime \prime}\right) 126.79$ $\left(\mathbf{C}^{\prime} 3^{\prime \prime \prime}\right) 122.51\left(\mathbf{C}-3^{\prime}\right) 121.04\left(\mathbf{C}^{\prime \prime}{ }^{\prime \prime}\right) 115.08\left(\mathbf{C}-3^{\prime \prime}, \mathbf{C}-5^{\prime \prime}\right) 105.42$ (C-4' a) $98.49\left(\mathbf{C - 8}^{\prime}\right) 92.96\left(\mathbf{C}^{\prime} \mathbf{6}^{\prime}\right) 71.13$ (C-1) 69.81 (C-2) 69.46 (C-3) $68.38\left(\mathbf{C}-4\right.$, C-5) $68.79\left(\mathbf{C}^{\prime \prime \prime \prime}\right) 61.80(\mathbf{C}-6) 28.42\left(\mathbf{C}^{\prime \prime}{ }^{\prime \prime \prime}\right)$ $20.45\left(\mathbf{C H}_{3}-\times 4\right)$.

$Z$ Isomer. ${ }^{1} \mathrm{H}$ NMR (600 MHz, DMSO-D6) $\delta$ ppm 12.96 (OH$\mathbf{5}^{\prime}$; s; 1H) $9.60\left(\mathbf{O H}-4^{\prime \prime}\right.$; s; $1 \mathrm{H}$; overlapped) $8.40\left(\mathbf{H}-\mathbf{2}^{\prime} ; \mathrm{s} ; 1 \mathrm{H}\right)$ 7.39 (H-2" $\mathbf{2}^{\prime \prime} \mathbf{H}-\mathbf{6}^{\prime \prime}$; $2 \mathrm{H}$; overlapped) 6.83 (H-3" ${ }^{\prime \prime}, \mathbf{H}-5^{\prime \prime} ; 2 \mathrm{H}$; overlapped) $6.69\left(\mathbf{H}^{\prime} \mathbf{8}^{\prime}\right.$; d; $\left.J_{5^{\prime}-7^{\prime}}=2.35 \mathrm{~Hz} ; 1 \mathrm{H}\right) 6.45\left(\mathbf{H}^{\prime} \mathbf{6}^{\prime}\right.$; $\left.\mathrm{d} ; J_{7^{\prime}-5^{\prime}}=2.35 \mathrm{~Hz} ; 1 \mathrm{H}\right) 5.76\left(\mathbf{H}-3^{\prime \prime \prime}\right.$; dt; $J_{3^{\prime \prime}-2^{\prime \prime}}=10.50 \mathrm{~Hz}$; $\left.J_{3^{\prime \prime}-4^{\prime \prime}}=5.40 \times 2 \mathrm{~Hz} ; 1 \mathrm{H}\right) 5.63\left(\mathbf{H}-2^{\prime \prime \prime}\right.$; ddd; $J_{2^{\prime \prime}-3^{\prime \prime}}=10.50 \mathrm{~Hz}$; 
$\left.J_{2^{\prime \prime}-1^{\prime \prime} \mathrm{b}}=6.80 \mathrm{~Hz} ; J_{2^{\prime \prime}-1^{\prime \prime} \mathrm{a}}=6.30 \mathrm{~Hz} ; 1 \mathrm{H}\right) 5.37\left(\mathbf{H}-3\right.$; dd; $J_{3-2}=$ $\left.9.10 \mathrm{~Hz} ; J_{3-4}=9.10 \mathrm{~Hz} ; 1 \mathrm{H}\right) 4.94\left(\mathbf{H}-2 ; \mathrm{dd} ; J_{2-3}=9.10 \mathrm{~Hz}\right.$; $\left.J_{2-1}=5.87 \mathrm{~Hz} ; 1 \mathrm{H}\right) 4.86(\mathbf{H}-4 ; 1 \mathrm{H}$; overlapped $) 4.80\left(\mathbf{H}-\mathbf{4}^{\prime \prime \prime}\right.$; d; $\left.J_{4^{\prime \prime}-3^{\prime \prime}}=5.40 \mathrm{~Hz} ; 2 \mathrm{H}\right) 4.21-4.13$ (H-1, H-6a; m; 2H) 4.00/3.93 $(\mathbf{H}-5, \mathbf{H}-6 \mathbf{b} ; \mathrm{m} ; 2 \mathrm{H}) 2.82\left(\mathbf{H}_{-1}^{\prime \prime \prime} \mathbf{a} ; \mathrm{m} ; J_{1^{\prime \prime} \mathrm{a}-1^{\prime \prime} \mathrm{b}}=15.26 \mathrm{~Hz}\right.$; $\left.J_{1^{\prime \prime} \mathrm{a}-2^{\prime \prime}}=9.39 \mathrm{~Hz} ; J_{1^{\prime \prime} \mathrm{a}-1}=9.39 \mathrm{~Hz} ; 1 \mathrm{H}\right) 2.38\left(\mathbf{H}^{-\mathbf{1}^{\prime \prime \prime}} \mathbf{b} ; \mathrm{m} ; 1 \mathrm{H}\right)$ $2.02 ; 2.01 ; 1.99 ; 1.98\left(\mathrm{CH}_{3}-; 12 \mathrm{H}\right)$.

${ }^{13} \mathrm{C}$ NMR (150 MHz, DMSO-D6) $\delta$ ppm: $180.37\left(\mathrm{C}^{\prime} \mathbf{4}^{\prime}\right.$; overlapped) $170-169\left(\mathbf{C}=\mathbf{O} \times 4, \mathbf{C}^{\prime} \mathbf{6}^{\prime}\right) 164.23\left(\mathbf{C}^{\prime} 5^{\prime}\right) 161.74$ (C-4 ${ }^{\prime \prime}$; overlapped) $157.42\left({\mathbf{C}-8^{\prime}}^{\prime}\right.$ a) 154.28 (C-2' ; overlapped) 130.13 (C-2 ${ }^{\prime \prime}, \mathbf{C}^{\prime} \mathbf{6}^{\prime \prime}$; overlapped) $129.98\left(\mathbf{C}-\mathbf{2}^{\prime \prime \prime}\right) 126.00\left(\mathbf{C}-3^{\prime \prime \prime}\right)$ 122.51 (C-3' ${ }^{\prime}$; overlapped) 121.04 (C-1' ${ }^{\prime \prime}$; overlapped) 115.08 (C$\mathbf{3}^{\prime \prime}, \mathbf{C}^{\prime \prime}{ }^{\prime \prime}$; overlapped) $105.42\left(\mathbf{C}-4^{\prime} \mathbf{a}\right.$; overlapped $) 98.49\left(\mathbf{C}-8^{\prime}\right.$; overlapped) 93.12 (C-6') 71.55 (C-1) 69.90 (C-2) 69.46 (C-3; overlapped) 68.38 (C-4, C-5; overlapped) $64.89\left(\mathbf{C}^{\prime \prime}{ }^{\prime \prime \prime}\right) 61.80$ (C-6; overlapped) $24.58\left({\left.\mathbf{C}-\mathbf{1}^{\prime \prime \prime}\right)}^{\prime \prime} 20.45\left(\mathbf{C H}_{3} \times 4\right.\right.$; overlapped).

HRMS $[\mathrm{M}+\mathrm{Na}]^{+}$. Experimental: 677.1821; calculated: 677.1841.

\subsection{3. $I^{\prime}, 4^{\prime}$-Bis-C-(2,3,4,6-tetra-O-acetyl- $\alpha$-D-glucopyrano- syl)-but-2'-en (4a)}

E Isomer. ${ }^{1} \mathrm{H} \mathrm{NMR}\left(300 \mathrm{MHz}, \mathrm{CDCl}_{3}\right) \delta$ ppm: $5.50\left(\mathbf{H}-\mathbf{2}^{\prime}, \mathbf{H}-\right.$ $\mathbf{3}^{\prime}$; t; $\left.3.8 \mathrm{~Hz} \times 2 ; 2 \mathrm{H}\right) 5.31\left(\mathbf{H}-3\right.$; dd; $J_{3-2}=9.3 \mathrm{~Hz} ; J_{3-4}=8.9 \mathrm{~Hz}$; 2H) $5.07\left(\mathbf{H}-2\right.$; dd; $\left.J_{2-3}=9.3 \mathrm{~Hz} ; J_{2-1}=5.4 \mathrm{~Hz} ; 2 \mathrm{H}\right) 5.00(\mathbf{H}-4$; dd; $\left.J_{4-3}=8.9 \mathrm{~Hz} ; J_{4-5}=8.9 \mathrm{~Hz} ; 2 \mathrm{H}\right) 4.26\left(\mathbf{H}-6 \mathrm{a} ; \mathrm{dd} ; J_{6 \mathrm{a}-6 \mathrm{~b}}=\right.$ $\left.12.2 \mathrm{~Hz} ; J_{6 \mathrm{a}-5}=5.0 \mathrm{~Hz} ; 2 \mathrm{H}\right) 4.21\left(\mathbf{H}-\mathbf{1} ; \mathrm{ddd} ; J_{1-1^{\prime} \mathrm{a}}=10.5 \mathrm{~Hz}\right.$, $\left.J_{1-2}=5.4 \mathrm{~Hz} ; J_{1-1^{\prime} \mathrm{b}}=4.9 \mathrm{~Hz} ; 2 \mathrm{H}\right) 4.06\left(\mathbf{H}-6 \mathbf{b} ; \mathrm{dd} ; J_{6 \mathrm{~b}-6 \mathrm{a}}=\right.$ $\left.12.2 \mathrm{~Hz} ; J_{6 \mathrm{~b}-5}=2.9 \mathrm{~Hz} ; 2 \mathrm{H}\right) 3.85\left(\mathbf{H}-5\right.$; ddd; $J_{5-4}=8.9 \mathrm{~Hz}$; $\left.J_{5-6 \mathrm{a}}=5.0 \mathrm{~Hz} ; J_{5-6 \mathrm{~b}}=2.9 \mathrm{~Hz} ; 2 \mathrm{H}\right) 2.52\left(\mathbf{H}_{-1}^{\prime} \mathbf{a}, \mathbf{H}-4^{\prime} \mathbf{a}\right.$; ddd; $\left.J_{1^{\prime} \mathrm{a}-1^{\prime} \mathrm{b}}=15.0 \mathrm{~Hz} ; J_{1^{\prime} \mathrm{a}-1}=10.5 \mathrm{~Hz} ; J=4.0 \mathrm{~Hz} ; 2 \mathrm{H}\right) 2.28\left(\mathbf{H}-\mathbf{1}^{\prime} \mathbf{b}\right.$, $\mathbf{H}-4^{\prime} \mathbf{b}$; ddd; $\left.J_{1^{\prime} \mathrm{b}-1^{\prime} \mathrm{a}}=15.0 \mathrm{~Hz} ; J_{1^{\prime} \mathrm{b}-1}=4.9 \mathrm{~Hz} ; J=4.0 \mathrm{~Hz} ; 2 \mathrm{H}\right)$ 2.09 (OAc; s; 6H) 2.06 (OAc; s; 6H) 2.04 (OAc; s; 12H).

${ }^{13} \mathrm{C} \mathrm{NMR}\left(75 \mathrm{MHz}, \mathrm{CDCl}_{3}\right) \delta \mathrm{ppm}: 170.62(\mathbf{C}=\mathbf{O}) 170.08$ $(\mathbf{C}=\mathbf{O}) 169.57(\mathbf{C}=\mathbf{O}) 169.46(\mathbf{C}=\mathbf{O}) 127.82\left(\mathbf{C}^{\prime} \mathbf{2}^{\prime}, \mathbf{C}-\mathbf{3}^{\prime}\right) 72.06$ (C-1) 70.19 (C-2) 70.06 (C-3) 68.87 (C-4) 68.60 (C-5) 62.07 (C-6) $29.33\left(\mathbf{C}-1^{\prime}, \mathbf{C}^{\prime} \mathbf{4}^{\prime}\right)$ 20.69/20.63 ( $\left.\mathbf{C H}_{3}\right)$.

$Z$ Isomer. ${ }^{1} \mathrm{H}$ NMR $\left(600 \mathrm{MHz}, \mathrm{CDCl}_{3}\right) \delta$ ppm: $5.52\left(\mathbf{H}-\mathbf{2}^{\prime}, \mathbf{H}-\right.$ $\left.3^{\prime} ; \mathrm{t} ; 4.4 \mathrm{~Hz} \times 2 ; 2 \mathrm{H}\right)$.

${ }^{13} \mathrm{C} \mathrm{NMR}\left(150 \mathrm{MHz}, \mathrm{CDCl}_{3}\right) \delta$ ppm: $170.40(\mathbf{C}=\mathbf{O}) 170.03$ $(\mathbf{C}=\mathbf{O}) 169.63(\mathbf{C}=\mathbf{O}) 69.53(\mathbf{C}=\mathbf{O}) 126.52\left(\mathbf{C}-2^{\prime}, \mathbf{C}^{\prime} \mathbf{3}^{\prime}\right) 72.06$ (C-1, overlapped) 70.19 (C-2, overlapped) 70.06 (C-3, overlapped) 69.17 (C-4) 68.70 (C-5) 61.99 (C-6) $24.56\left(\mathbf{C - 1}^{\prime}, \mathbf{C}^{\prime} \mathbf{4}^{\prime}\right)$ 20.69/20.63 $\left(\mathbf{C H}_{3}\right)$.

HRMS $[\mathrm{M}+\mathrm{Na}]^{+}$. Experimental: 739.2404; calculated: 739.2420 .

2.4.4. $7^{\prime}-O-\left(1^{\prime \prime \prime}-C-(2,3,4,6-T e t r a-O-a c e t y l-\alpha-D\right.$-galactopyranosyl)-but-2 $2^{\prime \prime \prime}-$ en $\left.-4^{\prime \prime \prime}-y l\right)$-genistein (3b)

E Isomer. ${ }^{1} \mathrm{H}$ NMR $\left(300 \mathrm{MHz}, \mathrm{CDCl}_{3}\right) \delta$ ppm: $12.81\left(\mathbf{H}-5^{\prime}\right.$;

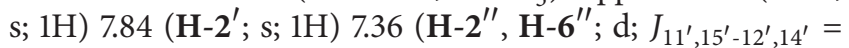

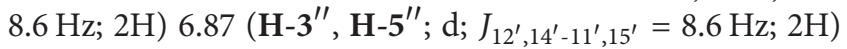
$6.37\left(\mathbf{H}-\mathbf{8}^{\prime} ; \mathrm{d} ; J_{5^{\prime}-7^{\prime}}=2.2 \mathrm{~Hz} ; 1 \mathrm{H}\right) 6.34\left(\mathbf{H}-\mathbf{6}^{\prime} ; \mathrm{d} ; J_{7^{\prime}-5^{\prime}}=2.2 \mathrm{~Hz}\right.$; 1H) $5.82 / 5.78\left(\mathbf{H}^{\prime \prime \prime}{ }^{\prime \prime}, \mathbf{H}-3^{\prime \prime \prime}\right.$; m; 2H) $5.44\left(\mathbf{H}-4 ; \mathrm{dd} ; J_{4-5}=\right.$
$\left.J_{4-3}=2.4 \mathrm{~Hz} ; 1 \mathrm{H}\right) 5.30 / 5.21(\mathbf{H}-2, \mathbf{H}-3 ; \mathbf{m} ; 2 \mathrm{H}) 4.52\left(\mathbf{H}^{\prime \prime} \mathbf{4}^{\prime \prime \prime} ; \mathrm{d}\right.$; $\left.J_{4^{\prime \prime}-3^{\prime \prime}}=3.7 \mathrm{~Hz} ; 2 \mathrm{H}\right) 4.36-4.28$ (H-1, H-6a; m; 2H) 4.13-4.05 (H-5, H-6b; m; 2H) 2.61-2.28 (H-1'"' a, H-1'"'b; m; 2H) 2.13 $\left(\mathbf{C H}_{3} ; \mathrm{s} ; 3 \mathrm{H}\right) 2.10\left(\mathbf{C H}_{3} ; \mathrm{s} ; 3 \mathrm{H}\right) 2.06\left(\mathbf{C H}_{3} ; \mathrm{s} ; 3 \mathrm{H}\right) 2.05\left(\mathbf{C H}_{3}\right.$; s; $3 \mathrm{H})$.

${ }^{13} \mathrm{C} \mathrm{NMR}\left(75 \mathrm{MHz}, \mathrm{CDCl}_{3}\right) \delta \mathrm{ppm}: 20.63\left(\mathbf{C H}_{3}\right) 20.72$ $\left(\mathbf{C H}_{3} \times 2\right) 20.76\left(\mathbf{C H}_{3}\right) 29.65\left(\mathbf{C}^{\prime \prime}{ }^{\prime \prime \prime}\right) 61.20(\mathbf{C}-6) 67.37(\mathbf{C}-5)$ $67.85\left(\mathbf{C}^{\prime \prime \prime}{ }^{\prime \prime \prime}\right) 68.26(\mathrm{C}-3) 68.49(\mathrm{C}-4) 68.70(\mathrm{C}-2) 71.19(\mathrm{C}-$ 1) $92.99\left(\mathbf{C}^{\prime} \mathbf{8}^{\prime}\right) 98.69\left(\mathbf{C}^{\prime} \mathbf{6}^{\prime}\right) 106.24\left(\mathbf{C}-\mathbf{4}^{\prime} \mathbf{a}\right) 115.59\left(\mathbf{C}-3^{\prime \prime}, \mathbf{C}-\right.$ $\left.5^{\prime \prime}\right) 122.58\left(\mathbf{C}^{\prime} \mathbf{3}^{\prime}\right) 123.66\left(\mathbf{C}^{\prime \prime} \mathbf{1}^{\prime \prime}\right) 126.97\left(\mathbf{C}-3^{\prime \prime \prime}\right) 130.16\left(\mathbf{C}-2^{\prime \prime \prime}\right)$ $130.24\left(\mathbf{C}-2^{\prime \prime}, \mathbf{C}^{\prime \prime} \mathbf{6}^{\prime \prime}\right) 152.76\left(\mathbf{C}-2^{\prime}\right) 156.28\left(\mathbf{C}-4^{\prime \prime}\right) 157.84(\mathrm{C}-$ $\mathbf{8}^{\prime}$ a) $162.56\left({\left.\mathbf{C}-5^{\prime}\right)}^{\prime} 164.36\left(\mathbf{C}-7^{\prime}\right) 169.92(\mathbf{C}=\mathbf{O}) 170.06(\mathbf{C}=\mathbf{O})\right.$ $170.14(\mathbf{C}=\mathbf{O}) 170.72(\mathbf{C}=\mathbf{O}) 180.86\left(\mathbf{C}-4^{\prime}\right)$.

$Z$ Isomer. ${ }^{1} \mathrm{H}$ NMR $\left(300 \mathrm{MHz}, \mathrm{CDCl}_{3}\right) \delta \mathrm{ppm}: 12.81(\mathbf{H}-$ $5^{\prime}$; overlapped; $\left.1 \mathrm{H}\right) 7.85\left(\mathbf{H}-\mathbf{2}^{\prime} ;\right.$ s; $\left.1 \mathrm{H}\right) 7.36\left(\mathbf{H}-\mathbf{2}^{\prime \prime}, \mathbf{H}-\mathbf{6}^{\prime \prime}\right.$; overlapped; $2 \mathrm{H}) 6.87\left(\mathbf{H}^{\prime \prime}{ }^{\prime \prime}, \mathbf{H}-\mathbf{5}^{\prime \prime}\right.$; overlapped; $\left.2 \mathrm{H}\right) 6.41(\mathbf{H}-$ $\mathbf{8}^{\prime}$; d; $\left.J_{5^{\prime}-7^{\prime}}=2.2 \mathrm{~Hz} ; 1 \mathrm{H}\right) 6.37\left(\mathbf{H}-\mathbf{6}^{\prime}\right.$; overlapped; $\left.1 \mathrm{H}\right) 5.87-$ $5.78\left(\mathbf{H}-3^{\prime \prime \prime} ; \mathrm{m} ; 1 \mathrm{H}\right) 5.71\left(\mathbf{H}-2^{\prime \prime \prime} ; \mathrm{dt} ; J_{2^{\prime \prime}-3^{\prime \prime}}=11.4 ; J_{2^{\prime \prime}-1^{\prime \prime}}=\right.$ $7.5 \times 2 \mathrm{~Hz} ; 1 \mathrm{H}) 5.44(\mathbf{H}-4$; overlapped; $1 \mathrm{H})$ 5.30-5.21 (H-2, H-3; overlapped; $2 \mathrm{H}) 4.62\left(\mathbf{H}-4^{\prime \prime \prime} ; \mathrm{d} ; J_{4^{\prime \prime}-3^{\prime \prime}}=5.9 \mathrm{~Hz} ; 2 \mathrm{H}\right)$ 4.36-4.28 (H-1, H-6a; overlapped; 2H) 4.13-4.05 (H-5, H-6b; overlapped; $2 \mathrm{H})$ 2.61-2.28 (H-1 ${ }^{\prime \prime \prime} \mathbf{a}, \mathbf{H}_{-1} \mathbf{1}^{\prime \prime} \mathbf{b}$; overlapped; $\left.2 \mathrm{H}\right)$ 2.13-2.05 ( $\mathrm{CH}_{3}$; overlapped; $\left.12 \mathrm{H}\right)$.

${ }^{13} \mathrm{C} \mathrm{NMR}\left(75 \mathrm{MHz}, \mathrm{CDCl}_{3}\right) \delta$ ppm: 20.63/20.76 $\left(\mathrm{CH}_{3}\right)$ $25.55\left(\mathbf{C}-1^{\prime \prime \prime}\right) 61.06(\mathbf{C}-6) 64.43\left(\mathbf{C}^{\prime \prime} 4^{\prime \prime \prime}\right) 67.25$ (C-5) 67.85 (C-3) 68.43 (C-4) 68.83 (C-2) 71.19 (C-1; overlapped) 92.99 (C-8' ; overlapped) $98.72\left(\mathbf{C - 6}^{\prime}\right) 106.30\left(\mathbf{C}-4^{\prime} \mathbf{a}\right) 115.59\left(\mathbf{C}-3^{\prime \prime}\right.$, $\mathbf{C}^{\prime \prime}{ }^{\prime \prime}$; overlapped) 122.58 (C-3'; overlapped) $123.69\left(\mathbf{C}-\mathbf{1}^{\prime \prime}\right)$ $126.25\left({\mathbf{C}-3^{\prime \prime \prime}}^{\prime \prime}\right) 129.47\left(\mathbf{C}-2^{\prime \prime \prime}\right) 130.24$ (C-2 $2^{\prime \prime}, \mathbf{C}^{\prime} \mathbf{6}^{\prime \prime}$; overlapped $)$ 152.76 (C-2' ; overlapped) 156.28 (C-4 ${ }^{\prime \prime}$; overlapped) 157.87 (C-8' a) $162.59\left({\left.\mathbf{C}-5^{\prime}\right)}^{\prime} 164.36\left({\mathbf{C}-7^{\prime}}^{\prime}\right.\right.$; overlapped) $169.92(\mathbf{C}=\mathbf{O}$; overlapped) $170.00(\mathbf{C}=\mathbf{O}) 170.10(\mathbf{C}=\mathbf{O}) 170.76(\mathbf{C}=\mathbf{O}) 180.86$ (C-4'; overlapped).

HRMS $[\mathrm{M}+\mathrm{Na}]^{+}$. Experimental: 677.1819; calculated: 677.1841.

\subsection{5. $I^{\prime}, 4^{\prime}$-Bis-C-(2,3,4,6-tetra-O-acetyl- $\alpha$-D-galactopyrano- syl)-but-2'-en $(\mathbf{4 b})$}

E Isomer. ${ }^{1} \mathrm{H} \mathrm{NMR}\left(300 \mathrm{MHz}, \mathrm{CDCl}_{3}\right) \delta$ ppm: $5.48\left(\mathbf{H}-\mathbf{2}^{\prime}, \mathbf{H}-\right.$ $\mathbf{3}^{\prime}$; t; $\left.3.7 \mathrm{~Hz} \times 2 ; 2 \mathrm{H}\right) 5.41\left(\mathbf{H}-\mathbf{4}\right.$; dd; $\left.J_{4-3}=J_{4-5}=2.7 \mathrm{~Hz} ; 2 \mathrm{H}\right)$ 5.26/5.22 (H-2; m; 2H) 5.19 (H-3; dd; $J_{3-4}=2.7$; $J_{3-2}=9.1 \mathrm{~Hz}$; 2H) 4.34/4.19 (H-1, H-6a; m; 4H) 4.11/4.02 (H-5, H-6b; m;

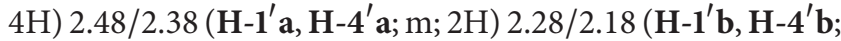
m; 4H) $2.12\left(\mathbf{C H}_{3} ; \mathrm{s} ; 6 \mathrm{H}\right) 2.08\left(\mathbf{C H}_{3} ; \mathrm{s} ; 6 \mathrm{H}\right) 2.06\left(\mathbf{C H}_{3} ; \mathrm{s} ; 6 \mathrm{H}\right)$ $2.04\left(\mathrm{CH}_{3} ; \mathrm{s} ; 6 \mathrm{H}\right)$.

${ }^{13} \mathrm{C}$ NMR (75 MHz, $\left.\mathrm{CDCl}_{3}\right) \delta$ ppm: $20.65\left(\mathbf{C H}_{3}\right) 20.73$

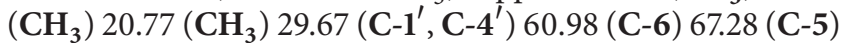
67.83 (C-3) 68.23 (C-2) 68.43 (C-4) 71.38 (C-1) 128.03 (C-2', \left.${\mathbf{C}-3^{\prime}}^{\prime}\right) 169.75(\mathbf{C}=\mathbf{O}) 169.89(\mathbf{C}=\mathbf{O}) 170.05(\mathbf{C}=\mathbf{O}) 170.51(\mathbf{C}=\mathbf{O})$.

$Z$ Isomer. ${ }^{1} \mathrm{H}$ NMR $\left(300 \mathrm{MHz}, \mathrm{CDCl}_{3}\right) \delta$ ppm: $5.51\left(\mathbf{H}-\mathbf{2}^{\prime}, \mathbf{H}-\right.$ $\mathbf{3}^{\prime}$; $\mathrm{t}$; $\left.4.4 \mathrm{~Hz} \times 2\right)$. Only $\mathbf{H}-\mathbf{2}^{\prime}$ and $\mathbf{H}-\mathbf{3}^{\prime}$ signals of $\mathrm{Z}$ isomer were not overlapped by signals of isomer $\mathrm{E}$.

${ }^{13} \mathrm{C} \mathrm{NMR}\left(75 \mathrm{MHz}, \mathrm{CDCl}_{3}\right) \delta$ ppm: 20.65/20.77 $\left(\mathbf{C H}_{3}\right)$ $24.81\left(\right.$ C-1 $\left.^{\prime}, \mathbf{C}^{\prime} \mathbf{4}^{\prime}\right) 61.13$ (C-6) 67.36 (C-5) 67.83 (C-3) 68.50 
(C-2) $68.64(\mathbf{C}-4) 71.38(\mathbf{C}-1) 126.77\left(\mathbf{C}^{\prime}{ }^{\prime}, \mathbf{C}^{\prime} \mathbf{3}^{\prime}\right) 169.85(\mathbf{C}=\mathbf{O}$ $\times 2) 170.02(\mathbf{C}=\mathbf{O}) 170.59(\mathbf{C}=\mathbf{O})$.

HRMS $[\mathrm{M}+\mathrm{Na}]^{+}$. Experimental: 739.2416; calculated: 739.2420 .

\subsection{6. $7^{\prime}-O-\left(1^{\prime \prime \prime}-C-(2,3,4,6-T e t r a-O-a c e t y l-\alpha-D\right.$-mannopyran-} osyl)-but-2"' -en-4'"'-yl)-genistein (3c)

E Isomer. ${ }^{1} \mathrm{H}$ NMR $\left(600 \mathrm{MHz}, \mathrm{d}_{6}\right.$-DMSO) $\delta$ ppm: $12.91\left(\mathbf{H}^{-5^{\prime}}\right.$; s; 1H) $9.62\left(\mathbf{H}^{\prime \prime}{ }^{\prime \prime}\right.$; s; 1H) $8.34\left(\mathbf{H}-\mathbf{2}^{\prime}\right.$; s; 1H) 7.37 (H-2 ${ }^{\prime \prime}, \mathbf{H}-\mathbf{6}^{\prime \prime}$; dd; $\left.J_{11^{\prime} 15^{\prime}-12^{\prime}, 14^{\prime}}=8.6 \mathrm{~Hz} ; J_{11^{\prime}-15^{\prime}}=2.2 \mathrm{~Hz} ; 2 \mathrm{H}\right) 6.81\left(\mathbf{H}-\mathbf{3}^{\prime \prime}, \mathbf{H}-\right.$

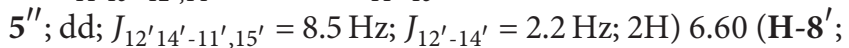
d; $\left.J_{5^{\prime}-7^{\prime}}=2.2 \mathrm{~Hz} ; 1 \mathrm{H}\right) 6.36\left(\mathbf{H}-\mathbf{6}^{\prime} ; \mathrm{d} ; J_{7^{\prime}-5^{\prime}}=2.2 \mathrm{~Hz} ; 1 \mathrm{H}\right) 5.84$ $\left(\mathbf{H}-3^{\prime \prime \prime} ; \mathrm{dt} ; J_{3^{\prime \prime}-2^{\prime \prime}}=15.5 \mathrm{~Hz} ; J_{3^{\prime \prime}-4^{\prime \prime}}=5.4 \times 2 \mathrm{~Hz} ; 1 \mathrm{H}\right) 5.79(\mathbf{H}-$ $2^{\prime \prime \prime}$; ddd; $J_{2^{\prime \prime}-3^{\prime \prime}}=15.5 \mathrm{~Hz} ; J_{2^{\prime \prime}-1^{\prime \prime} \mathrm{a}}=6.4 \mathrm{~Hz} ; J_{2^{\prime \prime}-1^{\prime \prime} \mathrm{b}}=6.4 \mathrm{~Hz}$; 1H) $5.24\left(\mathbf{H}-3\right.$; dd; $\left.J_{3-4}=9.0 \mathrm{~Hz} ; J_{3-2}=3.3 \mathrm{~Hz} ; 1 \mathrm{H}\right) 5.07(\mathbf{H}-2$; dd; $\left.J_{2-1}=3.3 \mathrm{~Hz} ; J_{2-3}=3.3 \mathrm{~Hz} ; 1 \mathrm{H}\right) 5.02\left(\mathbf{H}-4 ;\right.$ dd; $J_{4-5}=J_{4-3}=$ $8.5 \mathrm{~Hz} ; 1 \mathrm{H}) 4.61\left(\mathbf{H}^{\prime \prime \prime}{ }^{\prime \prime \prime} ; \mathrm{d} ; J_{4^{\prime \prime}-3^{\prime \prime}}=5.4 \mathrm{~Hz} ; 2 \mathrm{H}\right) 4.20(\mathbf{H}-\mathbf{6 a}$; dd; $\left.J_{6 \mathrm{a}-6 \mathrm{~b}}=12.2 \mathrm{~Hz} ; J_{6 \mathrm{a}-5}=6 . \mathrm{Hz} ; 1 \mathrm{H}\right) 3.99 / 3.93(\mathbf{H}-\mathbf{1}, \mathbf{H}-\mathbf{5}$, $\mathbf{H}-\mathbf{6 b} ; \mathbf{m} ; 3 \mathrm{H}) 2.69 / 2.59\left(\mathbf{H}_{-1} \mathbf{1}^{\prime \prime \prime} \mathbf{a} ; \mathbf{m}\right.$; $\left.\mathbf{1 H}\right) 2.43\left(\mathbf{H}-\mathbf{1}^{\prime \prime \prime} \mathbf{b}\right.$; ddd; $\left.J_{1^{\prime \prime} \mathrm{b}-1^{\prime \prime} \mathrm{a}}=14.6 \mathrm{~Hz} ; J_{1^{\prime \prime} \mathrm{b}-2^{\prime \prime}}=6.4 \mathrm{~Hz} ; J_{1^{\prime \prime} \mathrm{b}-1}=5.8 \mathrm{~Hz} ; 1 \mathrm{H}\right)$.

${ }^{13} \mathrm{C}$ NMR $\left(150 \mathrm{MHz}, \mathrm{d}_{6}\right.$-DMSO) $\delta \mathrm{ppm:} 20.46\left(\mathrm{CH}_{3}\right)$ $20.49\left(\mathbf{C H}_{3}\right) 20.54\left(\mathbf{C H}_{3}\right) 20.68\left(\mathbf{C H}_{3}\right) 31.26\left(\mathbf{C}-\mathbf{1}^{\prime \prime \prime}\right) 61.90(\mathbf{C}-$ 6) $64.90\left(\mathbf{C}-4^{\prime \prime \prime}\right) 66.66(\mathbf{C}-4) 68.30(\mathbf{C}-3) 69.79$ (C-2) 69.83 (C-5) $73.37(\mathbf{C}-1) 93.01\left(\mathbf{C}^{\prime} \mathbf{8}^{\prime}\right) 98.55\left(\mathbf{C}^{\prime} \mathbf{6}^{\prime}\right) 105.48\left(\mathbf{C}-\mathbf{4}^{\prime} \mathbf{a}\right)$ $115.14\left(\mathbf{C}-3^{\prime \prime}, \mathbf{C}-\mathbf{5}^{\prime \prime}\right) 121.10\left(\mathbf{C}-3^{\prime}\right) 122.56\left(\mathbf{C}-\mathbf{1}^{\prime \prime}\right) 127.09\left(\mathbf{C}-\mathbf{3}^{\prime \prime \prime}\right)$ $130.17\left(\mathbf{C}-2^{\prime \prime}, \mathbf{C}^{\prime \prime}{ }^{\prime \prime}, \mathbf{C}^{\prime \prime \prime \prime}\right) 154.28\left(\mathbf{C - 2} \mathbf{2}^{\prime}\right) 157.50\left(\mathbf{C}-\mathbf{4}^{\prime \prime}, \mathbf{C}^{\prime \prime} \mathbf{8}^{\prime} \mathbf{a}\right)$ $161.75\left(\mathbf{C}-5^{\prime}\right) 164.15\left(\mathbf{C}-7^{\prime}\right) 169.56(\mathbf{C}=\mathbf{O} \times 2) 169.77(\mathbf{C}=\mathbf{O})$ $170.08(\mathrm{C}=\mathbf{O}) 180.41\left(\mathbf{C}-\mathbf{4}^{\prime}\right)$.

$Z$ Isomer. ${ }^{1} \mathrm{H}$ NMR $\left(600 \mathrm{MHz}, \mathrm{CDCl}_{3}\right) \delta \mathrm{ppm}: 12.82\left(\mathbf{H}^{-5^{\prime}}\right.$; s; 1H) $7.85\left(\mathbf{H}^{\prime} \mathbf{2}^{\prime}\right.$; s; 1H) 7.39 (H-2 $\mathbf{2}^{\prime \prime}, \mathbf{H}-\mathbf{6}^{\prime \prime}$; d; $J_{11^{\prime}, 15^{\prime}-12^{\prime}, 14^{\prime}=}=$

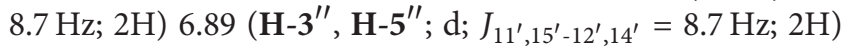
$6.43\left(\mathbf{H}-8^{\prime} ; \mathrm{d} ; J_{5^{\prime}-7^{\prime}}=2.4 \mathrm{~Hz} ; 1 \mathrm{H}\right) 6.40\left(\mathbf{H}^{\prime} \mathbf{6}^{\prime} ; \mathrm{d} ; J_{7^{\prime}-5^{\prime}}=2.4 \mathrm{~Hz}\right.$; 1H) $5.86\left(\mathbf{H}-3^{\prime \prime \prime} ;\right.$ ddd; $J_{3^{\prime \prime}-2^{\prime \prime}}=11.0 \mathrm{~Hz} ; J_{3^{\prime \prime}-4^{\prime \prime} \mathrm{a}}=J_{3^{\prime \prime}-4^{\prime \prime} \mathrm{b}}=$ $\left.6.3 \mathrm{~Hz} ; J_{3^{\prime \prime}-1^{\prime \prime} \mathrm{a}}=J_{3^{\prime \prime}-1^{\prime \prime} \mathrm{b}}=-1.4 \mathrm{~Hz} ; 1 \mathrm{H}\right) 5.73\left(\mathbf{H}^{2} \mathbf{2}^{\prime \prime \prime}\right.$; ddd; $J_{2^{\prime \prime}-3^{\prime \prime}}=11.0 \mathrm{~Hz} ; J_{2^{\prime \prime}-11^{\prime \prime} \mathrm{a}}=J_{2^{\prime \prime}-1^{\prime \prime} \mathrm{b}}=7.3 ; J_{2^{\prime \prime}-4^{\prime \prime} \mathrm{a}}=J_{2^{\prime \prime}-4^{\prime \prime} \mathrm{b}}=$ $-1.3 \mathrm{~Hz} ; 1 \mathrm{H}) 5.28\left(\mathrm{H}-3\right.$; dd; $\left.J_{3-4}=8.2 \mathrm{~Hz} ; J_{3-2}=3.4 \mathrm{~Hz} ; 1 \mathrm{H}\right) 5.20$ $\left(\mathbf{H}-2\right.$; dd; $\left.J_{2-1}=4.1 \mathrm{~Hz} ; J_{2-3}=3.4 \mathrm{~Hz} ; 1 \mathrm{H}\right) 5.18\left(\mathbf{H}-4\right.$; dd; $J_{4-3}=$ $\left.8.2 \mathrm{~Hz} ; J_{4-5}=7.6 \mathrm{~Hz} ; 1 \mathrm{H}\right) 4.68\left(\mathbf{H}-\mathbf{4}^{\prime \prime \prime}\right.$ a; ddd; $J_{4^{\prime \prime} \mathrm{a}-4^{\prime \prime} \mathrm{b}}=12.3 \mathrm{~Hz}$; $\left.J_{4^{\prime \prime} \mathrm{a}-3^{\prime \prime}}=6.3 \mathrm{~Hz} ; J_{4^{\prime \prime} \mathrm{a}-2^{\prime \prime}}=-1.3 \mathrm{~Hz} ; 1 \mathrm{H}\right) 4.65\left(\mathbf{H}-4^{\prime \prime \prime} \mathbf{b} ; \mathrm{ddd} ;\right.$ $\left.J_{4^{\prime \prime} \mathrm{b}-4^{\prime \prime} \mathrm{a}}=12.3 \mathrm{~Hz} ; J_{4^{\prime \prime} \mathrm{b}-3^{\prime \prime}}=6.3 \mathrm{~Hz} ; J_{4^{\prime \prime} \mathrm{b}-2^{\prime \prime}}=-1.3 \mathrm{~Hz} ; 1 \mathrm{H}\right) ; 4.42$ $\left(\mathbf{H}-6 \mathbf{6} ; \mathrm{dd} ; J_{6 \mathrm{a}-6 \mathrm{~b}}=12.1 \mathrm{~Hz} ; J_{6 \mathrm{a}-5}=6.7 \mathrm{~Hz} ; 1 \mathrm{H}\right) 4.12(\mathbf{H}-\mathbf{6 b} ; \mathrm{dd}$; $\left.J_{6 \mathrm{~b}-6 \mathrm{a}}=12.1 \mathrm{~Hz} ; J_{6 \mathrm{~b}-5}=3.3 \mathrm{~Hz} ; 1 \mathrm{H}\right) 4.06\left(\mathrm{H}-1 ; \mathrm{ddd} ; J_{1-1^{\prime \prime} \mathrm{a}}=8.7\right.$; $\left.J_{1-1^{\prime \prime} \mathrm{b}}=5.9 \mathrm{~Hz} ; J_{1-2}=4.1 \mathrm{~Hz} ; 1 \mathrm{H}\right) 2.58\left({\mathbf{H}-\mathbf{1}^{\prime \prime \prime}}^{\prime \prime} \mathbf{a}\right.$;ddd; $J_{1^{\prime \prime} \mathrm{a}-1^{\prime \prime} \mathrm{b}}=$ $\left.15.0 \mathrm{~Hz} ; J_{1^{\prime \prime} \mathrm{a}-1}=8.7 \mathrm{~Hz} ; J_{1^{\prime \prime} \mathrm{a}-2^{\prime \prime}}=7.3 \mathrm{~Hz} ; J_{1^{\prime \prime} \mathrm{a}-3^{\prime \prime}}=-1.4 \mathrm{~Hz} ; 1 \mathrm{H}\right)$ $2.53\left(\mathbf{H}-\mathbf{1}^{\prime \prime \prime} \mathrm{b}\right.$; dddd; $J_{1^{\prime \prime} \mathrm{a}-1^{\prime \prime} \mathrm{b}}=15.0 \mathrm{~Hz} ; J_{1^{\prime \prime} \mathrm{a}-1}=5.9 ; J_{1^{\prime \prime} \mathrm{a}-2^{\prime \prime}}=$ $\left.7.3 \mathrm{~Hz} ; J_{1^{\prime \prime} \mathrm{a}-3^{\prime \prime}}=-1.4 \mathrm{~Hz} ; 1 \mathrm{H}\right) 2.12\left(\mathbf{C H}_{3} ; \mathrm{s} ; 3 \mathrm{H}\right) 2.09\left(\mathbf{C H}_{3} ; \mathrm{s}\right.$; $6 \mathrm{H}) 2.06\left(\mathbf{C H}_{3} ; \mathrm{s} ; 3 \mathrm{H}\right)$.

${ }^{13} \mathrm{C}$ NMR $\left(150 \mathrm{MHz}, \mathrm{CDCl}_{3}\right) \delta \mathrm{ppm:} 20.74\left(\mathrm{CH}_{3}\right) 20.78$ $\left(\mathbf{C H}_{3}\right) 20.81\left(\mathbf{C H}_{3}\right) 20.93\left(\mathbf{C H}_{3}\right) 28.23\left(\mathbf{C}-\mathbf{1}^{\prime \prime \prime}\right) 62.05(\mathbf{C}-$ 6) $64.50\left(\mathrm{C}-4^{\prime \prime \prime}\right) 67.13(\mathrm{C}-4) 68.59(\mathrm{C}-3) 69.72(\mathrm{C}-2) 71.33$ (C-5) 73.33 (C-1) $93.10\left(\mathbf{C}^{\prime} \mathbf{8}^{\prime}\right) 98.81\left(\mathbf{C}-\mathbf{6}^{\prime}\right) 106.39\left(\mathbf{C}-\mathbf{4}^{\prime} \mathbf{a}\right)$ $115.60\left(\mathbf{C}-3^{\prime \prime}, \mathbf{C}^{\prime \prime} \mathbf{5}^{\prime \prime}\right) 122.95\left(\mathbf{C}-\mathbf{3}^{\prime}\right) 123.70\left(\mathbf{C}^{\prime \prime} \mathbf{1}^{\prime \prime}\right) 126.94(\mathbf{C}-$ $\left.\mathbf{3}^{\prime \prime \prime}\right) 128.69\left(\mathbf{C}^{\prime \prime \prime}{ }^{\prime \prime \prime}\right) 130.32\left(\mathbf{C}-2^{\prime \prime}, \mathbf{C}-\mathbf{6}^{\prime \prime}\right) 152.74\left(\mathbf{C}-\mathbf{2}^{\prime}\right) 156.04$ $\left(\mathbf{C}^{\prime \prime}{ }^{\prime \prime}\right) 157.92\left(\mathbf{C}^{\prime} \mathbf{8}^{\prime} \mathbf{a}\right) 162.73\left(\mathbf{C}-5^{\prime}\right) 164.43\left(\mathbf{C}^{\prime} \mathbf{7}^{\prime}\right) 169.70$
$(\mathbf{C}=\mathbf{O}) 169.96(\mathbf{C}=\mathbf{O}) 170.21(\mathbf{C}=\mathbf{O}) 170.72(\mathbf{C}=\mathbf{O}) 180.86(\mathbf{C}-$ $\left.4^{\prime}\right)$.

HRMS $[\mathrm{M}+\mathrm{Na}]^{+}$. Experimental: 677.1805; calculated: 677.1841.

2.4.7. $1^{\prime}, 4^{\prime}$-Bis-C-(2,3,4,6-tetra-O-acetyl- $\alpha$-D-mannopyranosyl)-but-2'-en (4c). Signals of $\mathrm{Z}$ isomer were completely overlapped by signals of isomer $\mathrm{E}$.

E Isomer. ${ }^{1} \mathrm{H}$ NMR $\left(300 \mathrm{MHz}, \mathrm{CDCl}_{3}\right) \delta$ ppm: $5.57\left(\mathbf{H}^{2} \mathbf{2}^{\prime}, \mathbf{H}-\right.$ $\mathbf{3}^{\prime}$; t; $3.3 \mathrm{~Hz} \times 2$; 2H) 5.27/5.17 (H-2, H-3, H-4; m; 6H) 4.34 $\left(\mathbf{H}-6 \mathbf{6} ; \mathrm{dd} ; J_{6 \mathrm{a}-6 \mathrm{~b}}=12.0 \mathrm{~Hz} ; J_{6 \mathrm{a}-5}=5.8 \mathrm{~Hz} ; 2 \mathrm{H}\right) 4.10(\mathbf{H}-\mathbf{- 6} \mathbf{b} ; \mathrm{dd} ;$ $\left.J_{6 \mathrm{~b}-6 \mathrm{a}}=12.0 \mathrm{~Hz} ; J_{6 \mathrm{~b}-5}=3.0 \mathrm{~Hz} ; 2 \mathrm{H}\right) 4.00\left(\mathbf{H}-\mathbf{1} ; \mathrm{ddd} ; J_{1-1^{\prime} \mathrm{a}}=\right.$ $\left.7.2 \mathrm{~Hz} ; J_{1-1^{\prime} \mathrm{a}}=7 . \mathrm{Hz} ; J_{1-1^{\prime} \mathrm{a}}=2.9 \mathrm{~Hz} ; 2 \mathrm{H}\right) 3.9\left(\mathbf{H}-5\right.$; ddd; $J_{5-4}$ $\left.=6.3 \mathrm{~Hz} ; J_{5-6 \mathrm{a}}=5.8 \mathrm{~Hz} ; J_{5-6 \mathrm{~b}}=3.0 \mathrm{~Hz} ; 2 \mathrm{H}\right) 2.54 / 2.36(\mathbf{H - 1} \mathbf{~} \mathbf{a}$, $\left.\mathbf{H}-\mathbf{1}^{\prime} \mathbf{b}, \mathbf{H}-\mathbf{4}^{\prime} \mathbf{a}, \mathbf{H}-\mathbf{4}^{\prime} \mathbf{b} ; \mathbf{m} ; 4 \mathrm{H}\right) 2.12\left(\mathbf{C H}_{3} ;\right.$ s; $\left.6 \mathrm{H}\right) 2.10\left(\mathbf{C H}_{3}\right.$; s; $6 \mathrm{H}) 2.07\left(\mathrm{CH}_{3} ; \mathrm{s} ; 6 \mathrm{H}\right) 2.03\left(\mathrm{CH}_{3} ; \mathrm{s} ; 6 \mathrm{H}\right)$.

${ }^{13} \mathrm{C}$ NMR $\left(75 \mathrm{MHz}, \mathrm{CDCl}_{3}\right) \delta$ ppm: $20.94\left(\mathrm{CH}_{3}\right) 20.77$ $\left(\mathbf{C H}_{3} \times 2\right) 20.70\left(\mathbf{C H}_{3}\right) 32.50\left(\mathbf{C}-\mathbf{1}^{\prime}, \mathbf{C}-\mathbf{4}^{\prime}\right) 62.34(\mathbf{C}-\mathbf{6}) 66.93$ (C-4) 68.69 (C-3) 69.82 (C-2) 70.72 (C-5) 74.29 (C-1) 128.00 $\left(\mathbf{C}-2^{\prime}, \mathbf{C}^{\prime} \mathbf{3}^{\prime}\right) 169.64(\mathrm{C}=\mathbf{O}) 169.95(\mathrm{C}=\mathbf{O}) 170.14(\mathrm{C}=\mathbf{O}) 170.66$ $(\mathrm{C}=\mathbf{O})$.

HRMS $[\mathrm{M}+\mathrm{Na}]^{+}$. Experimental: 739.2433; calculated: 739.2420 .

2.4.8. $7^{\prime}-O-\left(1^{\prime \prime \prime}-C-(2,3,4,6-T e t r a-O-b e n z y l-\alpha-D-g l u c o p y r a n o-\right.$ syl)-but-2'"'-en-4"'-yl)-genistein (3d)

E Isomer. ${ }^{1} \mathrm{H}$ NMR $\left(600 \mathrm{MHz}, \mathrm{CDCl}_{3}\right) \delta \mathrm{ppm}: 12.80\left(\mathbf{O H}-\mathbf{5}^{\prime}\right.$; s; 1H) 7.77 (H-2' ; s; 1H) 7.33/7.11 (-Ph, H-2" ${ }^{\prime \prime}$ H-6" ${ }^{\prime \prime}$; m; 22H)

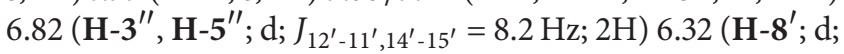
$\left.J_{5^{\prime}-7^{\prime}}=2.4 \mathrm{~Hz} ; 1 \mathrm{H}\right) 6.31\left(\mathbf{H}-\mathbf{6}^{\prime} ; \mathrm{d} ; J_{7^{\prime}-5^{\prime}}=2.4 \mathrm{~Hz} ; 1 \mathrm{H}\right) 5.83(\mathbf{H}-$ $\left.2^{\prime \prime \prime} ; \mathrm{d} ; J_{2^{\prime \prime}-3^{\prime \prime}}=15.6 ; J_{2^{\prime \prime}-1^{\prime \prime} \mathrm{a}}=7.0 \mathrm{~Hz} ; J_{2^{\prime \prime}-1^{\prime \prime} \mathrm{b}}=7.0 \mathrm{~Hz} ; 1 \mathrm{H}\right) 5.68$ $\left(\mathbf{H}-\mathbf{3}^{\prime \prime \prime} ; \mathrm{dt} ; J_{3^{\prime \prime}-2^{\prime \prime}}=15.6 \mathrm{~Hz} ; J_{3^{\prime \prime}-4^{\prime \prime}}=5.9 \mathrm{~Hz} \times 2 ; 1 \mathrm{H}\right) 4.94(-$ $\left.\mathbf{O C H}_{2} \mathbf{P h} ; \mathrm{d} ; J_{\mathrm{gem}}=10.9 ; 1 \mathrm{H}\right) 4.82\left(-\mathbf{O C H}_{2} \mathbf{P h} ; \mathrm{d} ; J_{\mathrm{gem}}=10.6\right.$; $1 \mathrm{H}) 4.81\left(-\mathbf{O C H}_{2} \mathbf{P h}\right.$; d; $\left.J_{\text {gem }}=10.9 \mathrm{~Hz} ; 1 \mathrm{H}\right) 4.70\left(-\mathbf{O C H}_{2} \mathbf{P h}\right.$; d; $\left.J_{\text {gem }}=11.7 \mathrm{~Hz} ; 1 \mathrm{H}\right) 4.61\left(-\mathbf{O C H}_{2} \mathbf{P h} ; \mathrm{d} ; J_{\text {gem }}=11.7 \mathrm{~Hz} ; 1 \mathrm{H}\right)$ $4.59\left(-\mathbf{O C H}_{2} \mathbf{P h} ; \mathrm{d} ; J_{\mathrm{gem}}=12.2 \mathrm{~Hz} ; \mathbf{1 H}\right) 4.47\left(-\mathbf{O C H}_{2} \mathbf{P h} ; \mathrm{d}\right.$; $\left.J_{\text {gem }}=10.6 \mathrm{~Hz} ; 1 \mathrm{H}\right) 4.46\left(-\mathbf{O C H}_{2} \mathbf{P h} ; \mathrm{d} ; J_{\text {gem }}=12.2 \mathrm{~Hz} ; 1 \mathrm{H}\right)$ $4.40\left(\mathbf{H}^{\prime}{ }^{\prime \prime \prime} ; \mathrm{d} ; J_{4^{\prime \prime}-3^{\prime \prime}}=5.9 \mathrm{~Hz} ; 2 \mathrm{H}\right) 4.13\left(\mathbf{H}-\mathbf{1} ; \mathrm{ddd} ; J_{1-2}=5.2\right.$; $\left.J_{1-1^{\prime \prime} \mathrm{a}}=10.6 \mathrm{~Hz} ; J_{1-1^{\prime \prime} \mathrm{b}}=5.0 \mathrm{~Hz} ; 1 \mathrm{H}\right) 3.81\left(\mathbf{H}-3 ; J_{3-2}=9.4 \mathrm{~Hz}\right.$; $\left.J_{3-4}=7.9 \mathrm{~Hz} ; 1 \mathrm{H}\right) 3.76\left(\mathrm{H}-2 ; \mathrm{dd} ; J_{2-3}=9.4 \mathrm{~Hz} ; J_{2-1}=5.7 \mathrm{~Hz}\right.$; 1H) 3.71-3.66 (H-6a; m; 1H) 3.65/3.59 (H-4, H-5, H-6b; m; 3H) $2.53\left(\mathbf{H}^{\prime \prime}{ }^{\prime \prime \prime} \mathbf{a}, \mathbf{H}-\mathbf{1}^{\prime \prime \prime} \mathbf{b} ; \mathbf{m} ; 2 \mathrm{H}\right)$.

${ }^{13} \mathrm{C}$ NMR $\left(150 \mathrm{MHz}, \mathrm{CDCl}_{3}\right) \delta \mathrm{ppm}: 180.79\left(\mathrm{C}^{\prime} \mathbf{4}^{\prime}\right) 164.53$ $\left(\mathbf{C}^{\prime} \mathbf{7}^{\prime}\right) 162.48\left(\mathbf{C}-8^{\prime} \mathbf{a}\right) 157.81\left(\mathbf{C}^{-5^{\prime}}\right) 156.21\left(\mathbf{C}^{\prime} \mathbf{4}^{\prime \prime}\right) 152.65\left(\mathbf{C}^{\prime} \mathbf{2}^{\prime}\right)$ 138.59 (Ph) 138.05 (Ph) 137.92 (Ph) 137.83 (Ph) $131.83\left(\mathbf{C}^{\prime \mathbf{2}^{\prime \prime \prime}}\right)$ $130.18\left({\mathbf{C}-2^{\prime \prime}}^{\prime \prime}, \mathbf{C}^{\prime \prime}{ }^{\prime \prime}\right) 128.45 / 127.60(\mathbf{P h} \times 4) 126.19\left(\mathbf{C}-3^{\prime \prime \prime}\right)$ $123.58\left(\mathbf{C}^{\prime} \mathbf{3}^{\prime}\right) 122.59\left(\mathbf{C}-\mathbf{1}^{\prime \prime}\right) 115.62\left(\mathbf{C}-\mathbf{3}^{\prime \prime}, \mathbf{C}^{\prime} \mathbf{5}^{\prime \prime}\right) 106.14\left(\mathbf{C}-\mathbf{4}^{\prime} \mathbf{a}\right)$ $98.82\left(\mathbf{C}^{\prime} \mathbf{6}^{\prime}\right) 92.95\left(\mathbf{C - 8}^{\prime}\right) 82.18$ (C-3) 79.90 (C-2) 78.07 (C4) $75.43\left(\mathbf{C H}_{2} \mathbf{P h}\right) 75.13\left(\mathbf{C H}_{2} \mathbf{P h}\right) 73.52(\mathbf{C}-1) 73.43\left(\mathbf{C H}_{2} \mathbf{P h}\right)$ 
$73.16\left(\mathrm{CH}_{2} \mathbf{P h}\right) 71.18(\mathrm{C}-5) 68.93(\mathrm{C}-6) 68.97\left(\mathrm{C}-4^{\prime \prime \prime}\right) 28.43(\mathrm{C}-$ $\left.1^{\prime \prime \prime}\right)$.

$Z$ Isomer. ${ }^{1} \mathrm{H}$ NMR $\left(600 \mathrm{MHz}, \mathrm{CDCl}_{3}\right) \delta$ ppm: $12.79\left(\mathbf{O H}^{\prime} \mathbf{5}^{\prime}\right.$; s; $1 \mathrm{H}) 7.77$ (H-2' $;$ s; $1 \mathrm{H}$; overlapped) $7.33 / 7.11$ (-Ph, H-2 ${ }^{\prime \prime}, \mathbf{H}-$ $\mathbf{6}^{\prime \prime} ; \mathrm{m} ; 22 \mathrm{H}$; overlapped) $6.82\left(\mathbf{H}^{-3^{\prime \prime}}, \mathbf{H}-\mathbf{5}^{\prime \prime} ; 2 \mathrm{H}\right.$; overlapped) $6.35\left(\mathbf{H}-\mathbf{8}^{\prime} ; \mathrm{d} ; J_{5^{\prime}-7^{\prime}}=2.35 \mathrm{~Hz} ; 1 \mathrm{H}\right) 6.34\left(\mathbf{H}-\mathbf{6}^{\prime} ; \mathrm{d} ; J_{7^{\prime}-5^{\prime}}=\right.$ $2.35 \mathrm{~Hz} ; 1 \mathrm{H}) 5.77\left(\mathbf{H}^{2} \mathbf{2}^{\prime \prime \prime}, \mathbf{H}-\mathbf{3}^{\prime \prime \prime} ; \mathrm{m} ; 2 \mathrm{H}\right) 4.93\left(-\mathbf{O C H}_{2} \mathbf{P h}\right.$; d; $\left.J_{\text {gem }}=10.86 \mathrm{~Hz} ; 1 \mathrm{H}\right) 4.82\left(-\mathbf{O C H}_{2} \mathbf{P h} ; \mathrm{d} ; J_{\text {gem }}=10.56 \mathrm{~Hz} ; 1 \mathrm{H}\right)$ $4.81\left(-\mathbf{O C H}_{2} \mathbf{P h} ; 1 \mathrm{H}\right.$; overlapped) $4.71\left(-\mathbf{O C H}_{2} \mathbf{P h} ; \mathrm{d} ; J_{\text {gem }}=\right.$ $11.44 \mathrm{~Hz} ; 1 \mathrm{H}) 4.60\left(-\mathbf{O C H}_{2} \mathbf{P h}\right.$; d $\left.J_{\text {gem }}=11.74 \mathrm{~Hz} ; 1 \mathrm{H}\right) 4.60$ $\left(-\mathbf{O C H}_{2} \mathbf{P h} ; \mathrm{d} ; J_{\text {gem }}=12.03 \mathrm{~Hz} ; 1 \mathrm{H}\right) 4.47\left(-\mathbf{O C H}_{2} \mathbf{P h} ; 1 \mathrm{H}\right.$; overlapped) $4.46\left(-\mathbf{O C H}_{2} \mathbf{P h} ; 1 \mathrm{H}\right.$; overlapped $) 4.58\left(\mathbf{H}-\mathbf{4}^{\prime \prime \prime}\right.$; $2 \mathrm{H}$; overlapped) $4.13\left(\mathbf{H}-\mathbf{1}\right.$; ddd; $J_{1-2}=5.21 ; J_{1-1^{\prime \prime} \mathrm{a}}=10.64 \mathrm{~Hz}$; $\left.J_{1-1{ }^{\prime \prime} \mathrm{b}}=5.0 \mathrm{~Hz} ; 1 \mathrm{H}\right) 3.81(\mathbf{H}-3 ; 1 \mathrm{H}$; overlapped) $3.76(\mathbf{H}-2$; $1 \mathrm{H}$; overlapped) $3.71 / 3.66(\mathbf{H}-6 \mathbf{a} ; 1 \mathrm{H}$; overlapped) $3.65 / 3.59$ (H-4, H-5, H-6b; 3H; overlapped) $2.53\left(\mathbf{H}_{-1}^{\prime \prime \prime} \mathbf{a}, \mathbf{H}-\mathbf{1}^{\prime \prime \prime} \mathbf{b} ; 2 \mathrm{H}\right.$; overlapped).

${ }^{13} \mathrm{C}$ NMR $\left(150 \mathrm{MHz}, \mathrm{CDCl}_{3}\right) \delta$ ppm: $180.83\left(\mathrm{C}-4^{\prime}\right) 164.51$ $\left(\mathbf{C}^{\prime} \mathbf{7}^{\prime}\right) 162.55\left(\mathbf{C}^{\prime} \mathbf{8}^{\prime} \mathbf{a}\right) 157.84\left(\mathbf{C}^{-5} \mathbf{5}^{\prime}\right) 156.16\left(\mathbf{C}^{\prime} \mathbf{4}^{\prime \prime}\right) 152.68\left(\mathbf{C}^{\prime} \mathbf{2}^{\prime}\right)$ 138.59-137.83 (Ph $\times 4$; overlapped) $130.94\left(\mathbf{C}-\mathbf{2}^{\prime \prime \prime}\right) 130.18(\mathbf{C}-$ $\mathbf{2}^{\prime \prime}, \mathbf{C}-\mathbf{6}^{\prime \prime}$; overlapped $)$ 128.45-127.60 (Ph $\left.\times 4\right) 125.51\left(\mathbf{C}-\mathbf{3}^{\prime \prime \prime}\right)$ $123.62\left(\mathbf{C}-3^{\prime}\right) 122.63\left(\mathbf{C}^{\prime \prime}{ }^{\prime \prime}\right) 115.62\left(\mathbf{C}-3^{\prime \prime}, \mathbf{C}^{\prime \prime} \mathbf{5}^{\prime \prime}\right.$; overlapped $)$ $106.23\left(\mathbf{C}^{\prime} \mathbf{4}^{\prime} \mathbf{a}\right) 98.83\left(\mathbf{C}^{\prime} \mathbf{6}^{\prime}\right) 92.98\left(\mathbf{C}-8^{\prime}\right) 82.12(\mathbf{C}-3) 79.82$ (C-2) $77.91(\mathbf{C}-4) 75.40\left(\mathbf{C H}_{2} \mathbf{P h}\right) 75.05\left(\mathbf{C H}_{2} \mathbf{P h}\right) 73.89(\mathbf{C}-\mathbf{1})$ $73.48\left(\mathbf{C H}_{2} \mathbf{P h}\right) 73.32\left(\mathrm{CH}_{2} \mathbf{P h}\right) 71.55(\mathbf{C}-5) 68.87(\mathbf{C}-6) 64.59$ $\left(\mathrm{C}-4^{\prime \prime \prime}\right) 24.14\left(\mathrm{C}-1^{\prime \prime \prime}\right)$.

\subsection{9. $1^{\prime}, 4^{\prime}$-Bis-C-(2,3,4,6-tetra-O-benzylo- $\alpha$-D-glukopyrano- syl)-but-2'-en (4d)}

E Isomer. ${ }^{1} \mathrm{H}$ NMR $\left(300 \mathrm{MHz}, \mathrm{CDCl}_{3}\right) \delta \mathrm{ppm}: 7.09 / 7.34$ (Ph; m; 40H) $5.51\left(\mathbf{H}^{\prime} \mathbf{2}^{\prime}, \mathbf{H}-\mathbf{3}^{\prime}\right.$; t; $3.5 \mathrm{~Hz} \times 2$; 2H) 4.94/4.41 $\left(\mathbf{C H}_{2} \mathbf{P h} ; \mathrm{m} ; 16 \mathrm{H}\right) 4.07\left(\mathbf{H}-\mathbf{1} ; \mathrm{ddd} ; J_{1-2}=5.6 \mathrm{~Hz} ; J_{1-1^{\prime} \mathrm{a}}=9.0 \mathrm{~Hz}\right.$; $\left.J_{1-1^{\prime} \mathrm{b}}=4.6 \mathrm{~Hz} ; 2 \mathrm{H}\right) 3.84 / 3.54(\mathbf{H}-\mathbf{2}, \mathbf{H}-3, \mathbf{H}-\mathbf{4}, \mathbf{H}-\mathbf{5}, \mathbf{H}-6 \mathbf{6}, \mathbf{H}-$ 6b; m; 12H) 2.50/2.41 (H-1' $\left.\mathbf{a}, \mathbf{H}-\mathbf{1}^{\prime} \mathbf{b}, \mathbf{H}-\mathbf{4}^{\prime} \mathbf{a}, \mathbf{H}-\mathbf{4}^{\prime} \mathbf{b} ; \mathrm{m} ; 4 \mathrm{H}\right)$.

$Z$ Isomer. ${ }^{1} \mathrm{H}$ NMR $\left(75 \mathrm{MHz}, \mathrm{CDCl}_{3}\right) \delta \mathrm{ppm}: 5.56\left(\mathbf{H}^{\prime} \mathbf{2}^{\prime}, \mathbf{H}-\right.$ $3^{\prime}$; t $; 4.0 \mathrm{~Hz} \times 2 ; 2 \mathrm{H}$ ). Only $\mathrm{H}-2^{\prime}$ and $\mathrm{H}-3^{\prime}$ signals were not overlapped by signals of $\mathrm{E}$ isomer.

\subsubsection{0. $7^{\prime}-O-\left(1^{\prime \prime \prime}-C-(3,4,6-T r i-O-a c e t y l-2-d e o x y-\alpha-D\right.$-glucopy- ranosyl)-but-2'"'-en- $\left.4^{\prime \prime \prime}-y l\right)$-genistein (3e)}

E Isomer. ${ }^{1} \mathrm{H}$ NMR $\left(300 \mathrm{MHz}, \mathrm{CDCl}_{3}\right) \delta \mathrm{ppm}: 12.81\left(\mathbf{H}-\mathbf{5}^{\prime}\right.$;

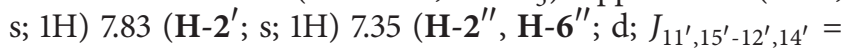

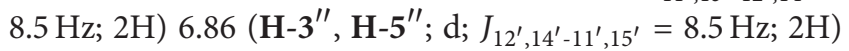
$6.37\left(\mathbf{H}-\mathbf{8}^{\prime} ; \mathrm{d} ; J_{5^{\prime}-7^{\prime}}=2.1 \mathrm{~Hz} ; 1 \mathrm{H}\right) 6.34\left(\mathbf{H}-\mathbf{6}^{\prime} ; \mathrm{d} ; J_{7^{\prime}-5^{\prime}}=2.1 \mathrm{~Hz}\right.$; 1H) $5.82\left(\mathbf{H}-2^{\prime \prime \prime}\right.$; dddt; $J_{2^{\prime \prime}-3^{\prime \prime}}=15.6 \mathrm{~Hz} ; J_{2^{\prime \prime}-1^{\prime \prime} \mathrm{a}}=J_{2^{\prime \prime}-1^{\prime \prime} \mathrm{b}}=$ $\left.7.1 \mathrm{~Hz} ; J_{2^{\prime \prime}-4^{\prime \prime}}=-1.5 \times 2 ; 1 \mathrm{H}\right) 5.79\left(\mathbf{H}-3^{\prime \prime \prime} ; \mathrm{dtd} ; J_{3^{\prime \prime}-2^{\prime \prime}}=15.6 \mathrm{~Hz}\right.$; $\left.J_{3^{\prime \prime}-4^{\prime \prime}}=5.4 \times 2 ; J_{3^{\prime \prime}-1^{\prime \prime} \mathrm{a}}=J_{3^{\prime \prime}-1{ }^{\prime \prime} \mathrm{b}}=-1.5 \mathrm{~Hz} ; 1 \mathrm{H}\right) 5.14(\mathbf{H}-3$; ddd; $\left.J_{3-2 \mathrm{~b}}=7.6 \mathrm{~Hz} ; J_{3-4}=7.0 \mathrm{~Hz} ; J_{3-2 \mathrm{a}}=4.6 \mathrm{~Hz} ; 1 \mathrm{H}\right) 4.89(\mathbf{H}-\mathbf{4} ; \mathrm{dd} ;$ $\left.J_{4-3}=J_{4-5}=7.0 \mathrm{~Hz} ; 1 \mathrm{H}\right) 4.51\left(\mathbf{H}-4^{\prime \prime \prime} ; \mathrm{d} ; J_{4^{\prime \prime}-3^{\prime \prime}}=3.7 \mathrm{~Hz} ; 2 \mathrm{H}\right) 4.43$ $\left(\mathbf{H}-6 \mathrm{a} ; \mathrm{dd} ; J_{6 \mathrm{a}-6 \mathrm{~b}}=12.1 \mathrm{~Hz} ; J_{6 \mathrm{a}-5}=6.2 \mathrm{~Hz} ; 1 \mathrm{H}\right) 4.12-4.06(\mathrm{H}-$ 1, H-6b; m; 2H) $3.94\left(\mathbf{H}-5\right.$; ddd; $J_{5-4}=7.0 \mathrm{~Hz} ; J_{5-6 \mathrm{a}}=6.2 \mathrm{~Hz}$; $\left.J_{5-6 \mathrm{~b}}=3.4 \mathrm{~Hz} ; 1 \mathrm{H}\right) 2.56\left({\mathbf{H}-\mathbf{1}^{\prime \prime \prime}}^{\prime \prime}\right.$; dddd; $J_{1^{\prime \prime} \mathrm{a}-1^{\prime \prime} \mathrm{b}}=-13.0 \mathrm{~Hz}$;
$\left.J_{1^{\prime \prime} \mathrm{a}-1}=8.5 ; J_{1^{\prime \prime} \mathrm{a}-2^{\prime \prime}}=7.1 \mathrm{~Hz} ; J_{1^{\prime \prime} \mathrm{a}-2^{\prime \prime}}=-1.5 \mathrm{~Hz} ; 1 \mathrm{H}\right) 2.34(\mathrm{H}-$ $\mathbf{1}^{\prime \prime \prime} \mathbf{b}$; dddd; $J_{1^{\prime \prime} \mathrm{b}-1^{\prime \prime} \mathrm{a}}=-13.0 \mathrm{~Hz} ; J_{1^{\prime \prime} \mathrm{b}-1}=5.2 \mathrm{~Hz} ; J_{1^{\prime \prime} \mathrm{b}-2^{\prime \prime}}=7.1 \mathrm{~Hz}$; $\left.J_{1^{\prime \prime} \mathrm{b}-3^{\prime \prime}}=-1.5 \mathrm{~Hz} ; 1 \mathrm{H}\right) 2.10\left(\mathbf{C H}_{3} ; \mathrm{s} ; 3 \mathrm{H}\right) 2.08\left(\mathbf{C H}_{3} ; \mathrm{s} ; 3 \mathrm{H}\right) 2.08$ $\left(\mathbf{C H}_{3} ;\right.$; $\left.3 \mathrm{H}\right) 2.00\left(\mathbf{H}-2 \mathbf{a} ;\right.$ ddd; $J_{2 \mathrm{a}-2 \mathrm{~b}}=14.0 \mathrm{~Hz} ; J_{2 \mathrm{a}-1}=4.8 \mathrm{~Hz}$; $\left.J_{2 \mathrm{a}-3}=4.6 \mathrm{~Hz} ; 1 \mathrm{H}\right) 1.90-1.82(\mathbf{H}-2 \mathbf{b} ; \mathrm{m} ; 1 \mathrm{H})$.

${ }^{13} \mathrm{C}$ NMR $\left(75 \mathrm{MHz}, \mathrm{CDCl}_{3}\right) \delta$ ppm: $20.77\left(\mathrm{CH}_{3}\right) 20.80$ $\left(\mathbf{C H}_{3}\right) 21.02\left(\mathbf{C H}_{3}\right) 31.97(\mathbf{C}-2) 35.32\left(\mathbf{C}^{\prime \prime} \mathbf{1}^{\prime \prime \prime}\right) 61.91(\mathbf{C}-\mathbf{6}) 68.36$ (C-4) 68.56 (C-3) 68.72 (C-4"') 69.42 (C-1) 70.91 (C-5) 93.04 $\left(\mathbf{C}^{\prime} \mathbf{8}^{\prime}\right) 98.70\left(\mathbf{C}^{\prime} \mathbf{6}^{\prime}\right) 106.19$ (C-4' a) $115.59\left(\mathbf{C}^{\prime} \mathbf{3}^{\prime \prime}, \mathbf{C}^{\prime} \mathbf{5}^{\prime \prime}\right) 122.47$ $\left(\mathbf{C}-3^{\prime}\right) 123.63\left(\mathbf{C}^{\prime \prime} \mathbf{1}^{\prime \prime}\right) 126.85\left(\mathbf{C}^{\prime \prime} \mathbf{3}^{\prime \prime \prime}\right) 130.63\left(\mathbf{C}^{\prime \prime} \mathbf{2}^{\prime \prime \prime}\right) 130.20(\mathbf{C}-$ $\left.\mathbf{2}^{\prime \prime}, \mathbf{C}^{\prime \prime} \mathbf{6}^{\prime \prime}\right) 152.74\left(\mathbf{C}^{\prime} \mathbf{2}^{\prime}\right) 156.34\left(\mathbf{C}-\mathbf{4}^{\prime \prime}\right) 157.81\left(\mathbf{C}-\mathbf{8}^{\prime}\right.$ a) $162.51(\mathbf{C}-$ $\left.5^{\prime}\right) 164.39\left(\mathbf{C}-7^{\prime}\right) 169.57(\mathbf{C}=\mathbf{O}) 170.22(\mathbf{C}=\mathbf{O}) 170.95(\mathbf{C}=\mathbf{O})$ $180.85\left(\mathbf{C}-4^{\prime}\right)$.

$Z$ Isomer. ${ }^{1} \mathrm{H}$ NMR $\left(300 \mathrm{MHz}, \mathrm{CDCl}_{3}\right) \delta \mathrm{ppm}: 12.81\left(\mathbf{H}-\mathbf{5}^{\prime}\right.$; s; 1H) $7.84\left(\mathbf{H}^{\prime} \mathbf{2}^{\prime}\right.$; s; 1H) 7.35 (H-2" ${ }^{\prime \prime} \mathbf{H}-\mathbf{6}^{\prime \prime}$; overlapped; $\left.2 \mathrm{H}\right)$ $6.86\left(\mathbf{H}-\mathbf{3}^{\prime \prime}, \mathbf{H}-\mathbf{5}^{\prime \prime}\right.$; overlapped; $\left.2 \mathrm{H}\right) 6.42\left(\mathbf{H}-\mathbf{8}^{\prime} ; \mathrm{d} ; J_{5^{\prime}-7^{\prime}}=\right.$ $2.1 \mathrm{~Hz} ; 1 \mathrm{H}) 6.38\left(\mathbf{H}-\mathbf{6}^{\prime}\right.$; d; $\left.J_{7^{\prime}-5^{\prime}}=2.1 \mathrm{~Hz} ; 1 \mathrm{H}\right) 5.85-5.79(\mathbf{H}-$ $\mathbf{3}^{\prime \prime \prime}$; overlapped; $\left.1 \mathrm{H}\right) 5.72\left(\mathbf{H}-2^{\prime \prime \prime}\right.$; ddd; $J_{2^{\prime \prime}-3^{\prime \prime}}=10.9 \mathrm{~Hz} ; J_{2^{\prime \prime}-1^{\prime \prime} \text { a }}$ $\left.=J_{2^{\prime \prime}-1{ }^{\prime \prime} \mathrm{b}}=7.3 \mathrm{~Hz} ; 1 \mathrm{H}\right) 5.14(\mathbf{H}-3$; overlapped; $1 \mathrm{H}) 4.88(\mathbf{H}-4$; dd; $\left.J_{4-3}=J_{4-5}=6.6 \mathrm{~Hz} ; 1 \mathrm{H}\right) 4.64\left(\mathrm{H}-4^{\prime \prime \prime} ; \mathrm{d} ; J_{4^{\prime \prime}-3^{\prime \prime}}=5.8 \mathrm{~Hz}\right.$; $2 \mathrm{H}) 4.48\left(\mathbf{H}-6 \mathrm{a} ; \mathrm{dd} ; J_{6 \mathrm{a}-6 \mathrm{~b}}=11.9 \mathrm{~Hz} ; J_{6 \mathrm{a}-5}=6.9 \mathrm{~Hz} ; 1 \mathrm{H}\right) 4.12-$ 4.06 (H-1, H-6b; m; 2H) 3.96 (H-5; partially overlapped; $1 \mathrm{H})$ 2.60-2.51 (H-1'"' a; overlapped; $1 \mathrm{H}) 2.40\left(\mathbf{H}-\mathbf{1}^{\prime \prime \prime} \mathbf{b}\right.$; dd; partially overlapped; $\left.J_{1^{\prime \prime} \mathrm{b}-2^{\prime \prime}}=7.3 ; J_{1^{\prime \prime} \mathrm{b}-1}=6.0 \mathrm{~Hz} ; 1 \mathrm{H}\right) 2.10-2.08\left(\mathbf{C H}_{3}\right.$; zakryte; 9H) 2.00 (H-2a; overlapped; $1 \mathrm{H})$ 1.90-1.82 (H-2b; overlapped; $1 \mathrm{H})$.

${ }^{13} \mathrm{C}$ NMR $\left(75 \mathrm{MHz}, \mathrm{CDCl}_{3}\right) \delta \mathrm{ppm}: 20.77\left(\mathrm{CH}_{3}\right.$; overlapped) $20.80\left(\mathbf{C H}_{3}\right.$; overlapped) $21.02\left(\mathbf{C H}_{3}\right.$; overlapped $)$ $31.13\left(\right.$ C-1 $\left.^{\prime \prime \prime}\right) 31.88$ (C-2) 61.78 (C-6) $64.47\left(\mathbf{C}^{\prime \prime} \mathbf{4}^{\prime \prime \prime}\right) 68.17$ (C-4) 68.52 (C-3) 69.20 (C-1) 71.22 (C-5) 93.04 (C-8' ; overlapped) $98.74\left(\mathbf{C}^{\prime} \mathbf{6}^{\prime}\right) 106.23\left(\mathbf{C}^{\prime} \mathbf{4}^{\prime} \mathbf{a}\right) 115.59$ (C-3"', C-5' 122.47 (C-3'; overlapped) 123.63 (C-1"' ; overlapped) 126.27 $\left(\mathbf{C}-3^{\prime \prime \prime}\right) 129.73\left(\mathbf{C - 2}^{\prime \prime \prime}\right) 130.20$ (C-2 ${ }^{\prime \prime}$, C-6"' ; overlapped) 152.74 (C-2'; overlapped) 156.34 (C-4"'; overlapped) $157.84\left(\mathbf{C}-\mathbf{8}^{\prime} \mathbf{a}\right)$ 162.51 (C-5' ${ }^{\prime}$; overlapped) $164.42\left(\mathbf{C}-7^{\prime}\right) 169.57(\mathbf{C}=\mathbf{O}$; overlapped) $170.22(\mathbf{C}=\mathbf{O}$; overlapped $) 170.95(\mathbf{C}=\mathbf{O}$; overlapped $)$ 180.85 (C-4'; overlapped).

HRMS $[\mathrm{M}+\mathrm{Na}]^{+}$. Experimental: 619.1772; calculated: 619.1786 .

\subsubsection{1. $1^{\prime}, 4^{\prime}$-Bis-C-(2,3,4,6-tetra-O-acetyl-2-deoxy- $\alpha$-D-gluco- pyranosyl)-but-2'-en $(\mathbf{4 e})$}

E Isomer. ${ }^{1} \mathrm{H}$ NMR $\left(300 \mathrm{MHz}, \mathrm{CDCl}_{3}\right) \delta$ ppm: $5.57\left(\mathbf{H}-\mathbf{2}^{\prime}, \mathbf{H}-\right.$ $\left.3^{\prime} ; \mathrm{m} ; 2 \mathrm{H}\right) 5.11\left(\mathbf{H}-3\right.$; ddd; $J_{3-4}=7.3 \mathrm{~Hz} ; J_{3-2 \mathrm{a}}=4.5 \mathrm{~Hz} ; J_{3-2 \mathrm{~b}}=$ $8.0 \mathrm{~Hz} ; 2 \mathrm{H}) 4.88\left(\mathbf{H}-\mathbf{4} ; \mathrm{dd} ; J_{4-5}=J_{4-3}=7.3 \mathrm{~Hz} ; 2 \mathrm{H}\right) 4.39(\mathbf{H}-\mathbf{6 a}$; dd; $\left.J_{6 \mathrm{a}-6 \mathrm{~b}}=12.0 \mathrm{~Hz} ; J_{6 \mathrm{a}-5}=5.9 \mathrm{~Hz} ; 2 \mathrm{H}\right) 4.07\left(\mathbf{H}-\mathbf{6 b} ; \mathrm{dd} ; J_{6 \mathrm{~b}-6 \mathrm{a}}=\right.$ $\left.12.0 \mathrm{~Hz} ; J_{6 \mathrm{~b}-5}=3.2 \mathrm{~Hz} ; 2 \mathrm{H}\right) 4.07 / 3.98(\mathbf{H}-\mathbf{1} ; \mathrm{m} ; 2 \mathrm{H}) 3.89$ (H-5; ddd; $\left.J_{5-4}=7.3 ; J_{5-6 \mathrm{a}}=5.9 ; J_{5-6 \mathrm{~b}}=3.2 \mathrm{~Hz} ; 2 \mathrm{H}\right) 2.51 / 2.22\left(\mathbf{H}-\mathbf{1}^{\prime} \mathbf{a}\right.$, $\mathbf{H}-\mathbf{1}^{\prime} \mathbf{b}, \mathbf{H}-\mathbf{4}^{\prime} \mathbf{a}, \mathbf{H}-\mathbf{4}^{\prime} \mathbf{b} ; \mathbf{m}$; 4H) $2.09\left(\mathbf{C H}_{3} ; \mathbf{s} ; 6 \mathrm{H}\right) 2.07\left(\mathbf{C H}_{3} ; \mathbf{s}\right.$; $6 \mathrm{H}) 2.06\left(\mathbf{C H}_{3} ; \mathrm{s} ; 6 \mathrm{H}\right) 2.02 / 1.93(\mathbf{H}-2 \mathrm{a} ; \mathrm{m} ; 2 \mathrm{H}) 1.90 / 1.79(\mathbf{H}-$ 2b; $\mathrm{m} ; 2 \mathrm{H})$.

${ }^{13} \mathrm{C}$ NMR $\left(75 \mathrm{MHz}, \mathrm{CDCl}_{3}\right) \delta$ ppm: $20.80\left(\mathrm{CH}_{3}\right) 20.83$

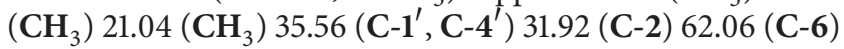


68.58 (C-4) 68.66 (C-3) 70.09 (C-1) 70.73 (C-5) 128.46 (C-2', $\left.\mathbf{C}^{-3^{\prime}}\right) 169.75(\mathbf{C}=\mathbf{O}) 170.03(\mathbf{C}=\mathbf{O}) 170.73(\mathbf{C}=\mathbf{O})$.

$Z$ Isomer. ${ }^{1} \mathrm{H}$ NMR $\left(300 \mathrm{MHz}, \mathrm{CDCl}_{3}\right) \delta$ ppm: $5.57\left(\mathbf{H}-\mathbf{2}^{\prime}, \mathbf{H}-\right.$ $3^{\prime}$; overlapped; $\left.2 \mathrm{H}\right) 5.14$ (H-3; partially overlapped; $\left.2 \mathrm{H}\right) 4.87$ $\left(\mathbf{H}-4\right.$; dd; $\left.J_{4-5}=J_{4-3}=7.0 \mathrm{~Hz} ; 2 \mathrm{H}\right) 4.42\left(\mathbf{H}-\mathbf{6 a} ; \mathrm{dd} ; J_{6 \mathrm{a}-6 \mathrm{~b}}=\right.$ $\left.12.0 \mathrm{~Hz} ; J_{6 \mathrm{a}-5}=6.4 \mathrm{~Hz} ; 2 \mathrm{H}\right) 4.1 / 4.05(\mathbf{H}-\mathbf{6 b}$; overlapped; $2 \mathrm{H})$ 4.07/3.98 (H-1; overlapped; $2 \mathrm{H}) 3.92\left(\mathbf{H}-5\right.$; ddd; $J_{5-4}=7.0$; $\left.J_{5-6 \mathrm{a}}=6.4 \mathrm{~Hz} ; J_{5-6 \mathrm{~b}}=3.4 \mathrm{~Hz} ; 2 \mathrm{H}\right) 2.51 / 2.22\left(\mathbf{H}^{\prime} \mathbf{1}^{\prime} \mathbf{a}, \mathbf{H}-\mathbf{1}^{\prime} \mathbf{b}\right.$, $\mathbf{H}-\mathbf{4}^{\prime} \mathbf{a}, \mathbf{H}-\mathbf{4}^{\prime} \mathbf{b}$; partially overlapped; $\left.4 \mathrm{H}\right) \mathbf{2 . 0 9 / 2 . 0 6}\left(\mathbf{C H}_{3}\right.$; overlapped; $18 \mathrm{H})$ 2.02/1.93 (H-2a; overlapped; $2 \mathrm{H})$ 1.90/1.79 (H-2b; overlapped; $2 \mathrm{H})$.

${ }^{13} \mathrm{C}$ NMR $\left(75 \mathrm{MHz}, \mathrm{CDCl}_{3}\right) \delta$ ppm: 20.80/21.04 $\left(\mathrm{CH}_{3}\right)$ $30.56\left(\mathbf{C}^{\prime} \mathbf{1}^{\prime}, \mathbf{C}^{\prime} \mathbf{4}^{\prime}\right) 32.00$ (C-2) 61.98 (C-6) 68.47 (C-4) $68.66(\mathrm{C}-3) 69.84(\mathrm{C}-1) 70.99$ (C-5) $127.10\left(\mathbf{C}^{2} \mathbf{2}^{\prime}, \mathrm{C}^{\prime} \mathbf{3}^{\prime}\right)$ $169.75 / 170.73(\mathrm{C}=\mathbf{O})$.

HRMS $[\mathrm{M}+\mathrm{Na}]^{+}$. Experimental: 623.2328; calculated: 623.2310 .

\subsubsection{2. $7^{\prime}-O-\left(1^{\prime \prime \prime}-C-(4-O-A c e t y l-2,3,6-t r i d e o x y-\alpha-L\right.$-erythro- heks-2-enpyranosyl)-but-2'"'-en-4'"'-yl)-genistein (3f)}

E Isomer. ${ }^{1} \mathrm{H}$ NMR $\left(600 \mathrm{MHz}, \mathrm{CDCl}_{3}\right) \delta$ ppm: $12.80(\mathbf{O H}-5$;

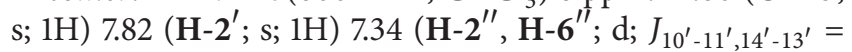

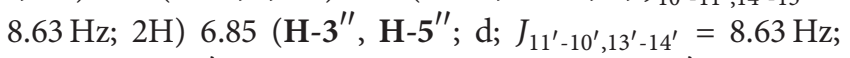
$2 \mathrm{H}) 6.36\left(\mathbf{H}-\mathbf{8}^{\prime} ; \mathrm{d} ; J_{7^{\prime}-5^{\prime}}=1.99 \mathrm{~Hz} ; 1 \mathrm{H}\right) 6.39\left(\mathbf{H}-\mathbf{6}^{\prime} ; \mathrm{d} ; J_{5^{\prime}-7^{\prime}}=\right.$ $2.65 \mathrm{~Hz} ; 1 \mathrm{H}) 5.92\left({\mathbf{H}-2^{\prime \prime \prime}}^{\prime \prime}\right.$;dd; $J_{2^{\prime \prime}-3^{\prime \prime}}=15.1 ; J_{2^{\prime \prime}-1^{\prime \prime} \mathrm{a}}=J_{2^{\prime \prime}-1^{\prime \prime} \mathrm{b}}=$ $7.3 \mathrm{~Hz} ; 1 \mathrm{H}) 5.94-5.92(\mathbf{H}-2 ; \mathrm{m} ; 1 \mathrm{H}) 5.82\left(\mathbf{H}-3\right.$; ddd; $J_{3-2}=$ $\left.10.1 \mathrm{~Hz} ; J_{3-4}=3.5 ; J_{3-1}=1.7 \mathrm{~Hz} ; 1 \mathrm{H}\right) 5.79\left(\mathbf{H}-\mathbf{3}^{\prime \prime \prime} ; \mathrm{dt} ; J_{3^{\prime \prime}-2^{\prime \prime}}=\right.$ $\left.15.1 \mathrm{~Hz} ; J_{3^{\prime \prime}-4^{\prime \prime}}=6.0 \mathrm{~Hz} \times 2 ; 1 \mathrm{H}\right) 4.91(\mathbf{H}-4$; broad signal; $1 \mathrm{H})$ $4.53\left(\mathbf{H}-\mathbf{4}^{\prime \prime \prime} ; \mathrm{d} ; J_{4^{\prime \prime}-3^{\prime \prime}}=6.0 \mathrm{~Hz} ; 2 \mathrm{H}\right) 4.26\left(\mathbf{H}-\mathbf{1} ; \mathrm{ddd} ; J_{1-1^{\prime \prime} \mathrm{a}}=\right.$ $\left.J_{1-1{ }^{\prime \prime} \mathrm{b}}=7.3 \mathrm{~Hz} ; J_{1-2}=1.99 \mathrm{~Hz} ; 1 \mathrm{H}\right) 3.96\left(\mathbf{H}-5\right.$; dq; $J_{5-6}=6.63 \mathrm{~Hz}$ $\left.\times 3 ; J_{5-4}=4.64 \mathrm{~Hz} ; 1 \mathrm{H}\right) 2.49\left(\mathbf{H}-\mathbf{1}^{\prime \prime \prime} \mathbf{a}\right.$; ddd; $J_{1^{\prime \prime} \mathrm{a}-1^{\prime \prime} \mathrm{b}}=14.60 \mathrm{~Hz}$; $\left.J_{1^{\prime \prime} \mathrm{a}-1}=J_{1^{\prime \prime} \mathrm{a}-2^{\prime \prime}}=7.30 \mathrm{~Hz} ; 1 \mathrm{H}\right) 2.38\left(\mathbf{H}-\mathbf{1}^{\prime \prime \prime} \mathbf{b} ;\right.$ ddd; $J_{1^{\prime \prime} \mathrm{b}-1^{\prime \prime} \mathrm{a}}=$ $\left.14.60 \mathrm{~Hz} ; J_{1^{\prime \prime} \mathrm{b}-1}=J_{1^{\prime \prime} \mathrm{b}-2^{\prime \prime}}=7.30 \mathrm{~Hz} ; 1 \mathrm{H}\right) 2.10(\mathbf{O A c} ; \mathrm{s} ; 3 \mathrm{H}) 1.26$ $\left(\mathrm{H}-6\right.$; $;$; $\left.J_{6-5}=6.63 \mathrm{~Hz} \times 3 ; 3 \mathrm{H}\right)$.

${ }^{13} \mathrm{C}$ NMR $\left(75 \mathrm{MHz}, \mathrm{CDCl}_{3}\right) \delta \mathrm{ppm}: 180.87\left(\mathrm{C}-4^{\prime}\right) 170.07$ $(\mathbf{C}=\mathbf{O}) 164.46\left(\mathbf{C}^{\prime} \mathbf{7}^{\prime}\right) 162.42\left(\mathbf{C}-5^{\prime}\right) 157.81\left(\mathbf{C}-4^{\prime \prime}\right) 156.39(\mathbf{C}-$ $\left.\mathbf{8}^{\prime} \mathbf{a}\right) 152.77\left(\mathbf{C}-\mathbf{2}^{\prime}\right) 33.20(\mathbf{C}-2) 131.38\left(\mathbf{C}^{\prime 2} \mathbf{2}^{\prime \prime \prime}\right) 130.15\left(\mathbf{C}-\mathbf{2}^{\prime \prime}\right.$, C6") $^{\prime \prime} 126.60\left(\mathbf{C}-3^{\prime \prime \prime}\right) 123.65\left(\mathbf{C}-3^{\prime}\right) 123.06(\mathbf{C}-3) 122.30\left(\mathbf{C}^{\prime \prime}{ }^{\prime \prime}\right)$ $115.64\left(\mathbf{C}^{\prime \prime}{ }^{\prime \prime}, \mathbf{C}^{\prime} \mathbf{5}^{\prime \prime}\right) 106.14\left(\mathbf{C}-\mathbf{4}^{\prime} \mathbf{a}\right) 98.78\left(\mathbf{C}^{\prime} \mathbf{8}^{\prime}\right) 93.02\left(\mathbf{C}^{\prime \prime} \mathbf{6}^{\prime}\right)$ 69.53 (C-1) 69.36 (C-4) $68.88\left(\right.$ C-5, C-4 $\left.{ }^{\prime \prime \prime}\right) 36.74\left(\right.$ C-1 $\left.^{\prime \prime \prime}\right) 21.19$ $\left(\mathrm{CH}_{3}{ }^{-}\right) 16.74(\mathbf{C}-6)$.

$Z$ Isomer. ${ }^{1} \mathrm{H}$ NMR $\left(600 \mathrm{MHz}, \mathrm{CDCl}_{3}\right) \delta \mathrm{ppm}: 12.81\left(\mathbf{O H}-\mathbf{5}^{\prime}\right.$; s; $1 \mathrm{H}) 7.84\left(\mathbf{H}^{\prime} \mathbf{2}^{\prime}\right.$; $;$; 1H) $7.34\left(\mathbf{H}^{\prime \prime} \mathbf{2}^{\prime \prime}, \mathbf{H}-\mathbf{6}^{\prime \prime}\right.$; overlapped; $\left.2 \mathrm{H}\right) 6.85$ $\left(\mathbf{H}-3^{\prime \prime}, \mathbf{H}-5^{\prime \prime}\right.$; overlapped; $\left.2 \mathrm{H}\right) 6.40\left(\mathbf{H}-\mathbf{8}^{\prime} ; \mathrm{d} ; J_{5^{\prime}-7^{\prime}}=2.32 \mathrm{~Hz}\right.$; 1H) $6.37\left(\mathbf{H}^{\prime} \mathbf{6}^{\prime}\right.$; d; $\left.J_{7^{\prime}-5^{\prime}}=2.3 \mathrm{~Hz} ; 1 \mathrm{H}\right) 5.94\left(\mathbf{H - 2}\right.$; ddd; $J_{2-3}=$ $\left.10.1 \mathrm{~Hz} ; J_{2-1}=2.7 \mathrm{~Hz} ; J_{2-4}=-1.3 \mathrm{~Hz}\right) 5.85\left(\mathbf{H}-3\right.$; ddd; $J_{3-2}=$ $\left.10.1 \mathrm{~Hz} ; J_{3-4}=3.5 \mathrm{~Hz} ; J_{3-1}=-1.7 \mathrm{~Hz} ; 1 \mathrm{H}\right) 5.80 / 5.83\left({\mathbf{H}-\mathbf{2}^{\prime \prime \prime}}^{\prime \prime} ; \mathbf{H}-\right.$ $3^{\prime \prime \prime}$; overlapped; $\left.2 \mathrm{H}\right) 4.91(\mathbf{H}-4$; overlapped; $1 \mathrm{H}) 4.65\left(\mathbf{H}-\mathbf{4}^{\prime \prime \prime}\right.$; d; $\left.J_{4^{\prime \prime}-3^{\prime \prime}}=4.6 \mathrm{~Hz} ; 2 \mathrm{H}\right) 4.26(\mathbf{H}-\mathbf{1}$; overlapped; $1 \mathrm{H}) 3.96(\mathbf{H}-5$; overlapped; 1H) $2.49\left(\mathbf{H}-\mathbf{1}^{\prime \prime \prime} \mathbf{a}\right.$; overlapped; $\left.1 \mathrm{H}\right) 2.44\left(\mathbf{H}-\mathbf{1}^{\prime \prime \prime} \mathbf{b}\right.$; ddd; $\left.J_{1^{\prime \prime} \mathrm{b}-1^{\prime \prime} \mathrm{a}}=14.60 \mathrm{~Hz} ; J_{1^{\prime \prime} \mathrm{b}-1}=J_{1^{\prime \prime} \mathrm{b}-2^{\prime \prime}}=6.0 \mathrm{~Hz} ; 1 \mathrm{H}\right) 2.09$ (OAc; s; $3 \mathrm{H}) 1.27\left(\mathbf{H}-6\right.$; t $\left.; J_{6-5}=6.63 \mathrm{~Hz} \times 3 ; 3 \mathrm{H}\right)$.

${ }^{13} \mathrm{C}$ NMR $\left(75 \mathrm{MHz}, \mathrm{CDCl}_{3}\right) \delta \mathrm{ppm}: 180.87\left(\mathrm{C}-4^{\prime}\right.$; overlapped) 170.07 (C=O; overlapped) $164.46\left(\mathbf{C}-\mathbf{7}^{\prime}\right.$; overlapped $)$
$162.46\left(\mathbf{C}-5^{\prime}\right) 157.81$ (C-4"'; overlapped) $156.39\left(\mathbf{C}-8^{\prime} \mathbf{a}\right.$; overlapped) 152.77 (C-2' ; overlapped) 133.09 (C-2) 130.15 (C-2'", $\mathbf{C}^{\prime \prime}{ }^{\prime \prime}, \mathbf{C}-\mathbf{6}^{\prime \prime}$; overlapped) $126.16\left(\mathbf{C}-\mathbf{3}^{\prime \prime \prime}\right) 123.68\left(\mathbf{C}-\mathbf{3}^{\prime}\right) 123.23$ (C-3) 122.30 (C-1" ; overlapped) $115.64\left(\mathbf{C}-\mathbf{3}^{\prime \prime}, \mathbf{C}-5^{\prime \prime}\right.$; overlapped) $106.17\left(\mathbf{C}^{\prime} \mathbf{4}^{\prime} \mathbf{a}\right) 98.72\left(\mathbf{C}^{\prime} \mathbf{8}^{\prime}\right) 93.02\left(\mathbf{C}^{\prime} \mathbf{6}^{\prime}\right.$; overlapped) 69.31 (C-1) 69.27 (C-4) 69.07 (C-5) $64.54\left(\mathbf{C}-4^{\prime \prime \prime}\right) 32.43$ (C$\left.\mathbf{1}^{\prime \prime \prime}\right) 21.19\left(\mathbf{C H}_{3}-\right.$; overlapped) 16.74 (C-6; overlapped).

LRMS $[\mathrm{M}+\mathrm{Na}]^{+}$. Experimental: 501.2; calculated: 501.2.

2.4.13. $I^{\prime}, 4^{\prime}$-Bis-C-(4-O-acetyl-2,3,6-trideoxy- $\alpha$-L-erythroheks-2-enpyranosyl)-but-2'-en (4f)

E Isomer. ${ }^{1} \mathrm{H}$ NMR $\left(600 \mathrm{MHz}, \mathrm{CDCl}_{3}\right) \delta \mathrm{ppm}: 5.92(\mathbf{H}-2$; ddd; $\left.J_{2-3}=10.2 \mathrm{~Hz} ; J_{2-1}=2.2 \mathrm{~Hz} ; J_{2-4}=1.5 \mathrm{~Hz} ; 2 \mathrm{H}\right) 5.77(\mathrm{H}-$ 3; ddd; $J_{3-2}=10.2 \mathrm{~Hz} ; J_{3-4}=3.4 \mathrm{~Hz}$; $\left.J_{3-1}=2.2 \mathrm{~Hz} ; 2 \mathrm{H}\right) 5.58$ $\left(\mathbf{H}-2^{\prime}\right.$; ddddd; $J_{2^{\prime}-3^{\prime}}=15.5 \mathrm{~Hz} ; J_{2^{\prime}-1^{\prime} \mathrm{a}}=6.5 \mathrm{~Hz} ; J_{2^{\prime}-1^{\prime} \mathrm{b}}=7.5 \mathrm{~Hz}$; $\left.J_{2^{\prime}-4^{\prime} \mathrm{a}}=J_{2^{\prime}-4^{\prime} \mathrm{b}}=-1.5 \mathrm{~Hz} ; 1 \mathrm{H}\right) 5.58\left(\mathbf{H}-3^{\prime}\right.$; ddddd; $J_{3^{\prime}-2^{\prime}}=$ $15.5 \mathrm{~Hz} ; J_{3^{\prime}-4^{\prime} \mathrm{a}}=6.5 \mathrm{~Hz} ; J_{3^{\prime}-4^{\prime} \mathrm{b}}=7.5 \mathrm{~Hz} ; J_{3^{\prime}-1^{\prime} \mathrm{a}}=J_{3^{\prime}-1^{\prime} \mathrm{b}}=$ $-1.5 \mathrm{~Hz} ; 1 \mathrm{H}) 4.88\left(\mathbf{H}-4\right.$; dddd; $J_{4-5}=4.7 \mathrm{~Hz} ; J_{4-3}=3.4 \mathrm{~Hz}$; $\left.J_{4-2}=1.4 \mathrm{~Hz} ; J_{4-1}=2.2 \mathrm{~Hz} ; 2 \mathrm{H}\right) 4.17\left(\mathbf{H}-\mathbf{1} ;\right.$ ddddd; $J_{1-1^{\prime} \mathrm{a}}=$ $\left.9.0 \mathrm{~Hz} ; J_{1-1^{\prime} \mathrm{b}}=6.7 \mathrm{~Hz} ; J_{1-2}=J_{1-3}=J_{1-4}=2.2 \mathrm{~Hz} ; 2 \mathrm{H}\right) 3.91(\mathrm{H}-$ 5; dq; $\left.J_{5-6}=6.6 \mathrm{~Hz} \times 3 ; J_{5-4}=4.7 \mathrm{~Hz} ; 2 \mathrm{H}\right) 2.46 / 2.40\left(\mathbf{H}-\mathbf{1}^{\prime} \mathbf{a}\right.$, $\mathbf{H}-\mathbf{4}^{\prime} \mathbf{a} ; \mathbf{m}$; $\left.2 \mathrm{H}\right) 2.28\left(\mathbf{H}-\mathbf{1}^{\prime} \mathbf{b}\right.$; dddd; $J_{1^{\prime} \mathbf{b}-1^{\prime} \mathbf{a}}=14.0 \mathrm{~Hz} ; J_{1^{\prime} \mathbf{b}-2^{\prime}}=$ $\left.7.5 \mathrm{~Hz} ; J_{1^{\prime} \mathrm{b}-1}=6.7 \mathrm{~Hz} ; J_{1^{\prime} \mathrm{b}-3^{\prime}}=-1.5 \mathrm{~Hz} ; 1 \mathrm{H}\right) 2.28\left(\mathbf{H}-\mathbf{4}^{\prime} \mathbf{b}\right.$; dddd; $J_{4^{\prime} \mathrm{b}-4^{\prime} \mathrm{a}}=14.0 \mathrm{~Hz} ; J_{4^{\prime} \mathrm{b}-3^{\prime}}=7.5 \mathrm{~Hz} ; J_{4^{\prime} \mathrm{b}-1}=6.7 \mathrm{~Hz} ; J_{4^{\prime} \mathrm{b}-2^{\prime}}=$ $-1.5 \mathrm{~Hz}$; $1 \mathrm{H}) 2.09\left(\mathrm{CH}_{3}\right.$; s; 6H) $1.23\left(\mathbf{H}-6\right.$; d; $\left.J_{6-5}=6.6 ; 6 \mathrm{H}\right)$.

${ }^{13} \mathrm{C}$ NMR $\left(150 \mathrm{MHz}, \mathrm{CDCl}_{3}\right) \delta \mathrm{ppm}: 16.92(\mathbf{C}-6) 21.18$ $\left(\mathbf{C H}_{3}\right) 37.17\left(\mathbf{C}-1^{\prime}, \mathbf{C}-4^{\prime}\right) 68.62(\mathbf{C}-4) 69.57(\mathbf{C}-5) 70.13(\mathbf{C}-1)$ $122.94(\mathbf{C}-3) 128.66\left(\mathbf{C}^{\prime} \mathbf{2}^{\prime}, \mathbf{C}^{\prime} \mathbf{3}^{\prime}\right) 133.46(\mathbf{C}-2) 170.69(\mathbf{C}=\mathbf{O})$.

$Z$ Isomer. ${ }^{1} \mathrm{H}$ NMR $\left(600 \mathrm{MHz}, \mathrm{CDCl}_{3}\right) \delta$ ppm: 5.93 (H-2; ddd; $\left.J_{2-3}=10.2 \mathrm{~Hz} ; J_{2-1}=2.2 \mathrm{~Hz} ; J_{2-4}=1.4 \mathrm{~Hz} ; 2 \mathrm{H}\right) 5.79(\mathbf{H}-3$; ddd; $\left.J_{3-2}=10.2 \mathrm{~Hz} ; J_{3-4}=3.5 \mathrm{~Hz} ; J_{3-1}=2.2 \mathrm{~Hz} ; 2 \mathrm{H}\right) 5.61(\mathrm{H}-$ $2^{\prime}$; ddddd; $J_{2^{\prime}-3^{\prime}}=11.0 \mathrm{~Hz} ; J_{2^{\prime}-1^{\prime} \mathrm{a}}=6.5 ; J_{2^{\prime}-1^{\prime} \mathrm{b}}=7.5 \mathrm{~Hz} ; J_{2^{\prime}-4^{\prime} \mathrm{a}}=$ $J_{2^{\prime}-4^{\prime} \mathrm{b}}=-1.6 \mathrm{~Hz}$; $\left.1 \mathrm{H}\right) 5.61\left(\mathbf{H}-3^{\prime}\right.$; ddddd; $J_{3^{\prime}-2^{\prime}}=11.0 \mathrm{~Hz} ; J_{3^{\prime}-4^{\prime} \mathrm{a}}$ $\left.=6.5 \mathrm{~Hz} ; J_{3^{\prime}-4^{\prime} \mathrm{b}}=7.5 \mathrm{~Hz} ; J_{3^{\prime}-1^{\prime} \mathrm{a}}=J_{3^{\prime}-1^{\prime} \mathrm{b}}=-1.6 \mathrm{~Hz} ; 1 \mathrm{H}\right) 4.88$ $\left(\mathbf{H}-4\right.$; dddd; $J_{4-5}=4.9 \mathrm{~Hz} ; J_{4-3}=3.5 \mathrm{~Hz} ; J_{4-2}=1.4 \mathrm{~Hz} ; J_{4-1}=$ $2.2 \mathrm{~Hz} ; 2 \mathrm{H}) 4.19\left(\mathrm{H}-1\right.$; ddddd; $J_{1-1^{\prime} \mathrm{a}}=9.0 \mathrm{~Hz} ; J_{1-1^{\prime} \mathrm{b}}=6.7 \mathrm{~Hz}$; $\left.J_{1-2}=J_{1-3}=J_{1-4}=2.2 \mathrm{~Hz} ; 2 \mathrm{H}\right) 3.92\left(\mathrm{H}-5 ; \mathrm{dq} ; J_{5-6}=6.6 \mathrm{~Hz} \times 3\right.$; $\left.J_{5-4}=4.9 \mathrm{~Hz} ; 2 \mathrm{H}\right) 2.46 / 2.40\left(\mathbf{H}^{-1} \mathbf{l}^{\prime} \mathbf{a}, \mathbf{H}-\mathbf{4}^{\prime} \mathbf{a}\right.$; overlapped; $\left.2 \mathrm{H}\right)$ $2.34\left(\mathbf{H}-\mathbf{1}^{\prime} \mathbf{b}\right.$; dddd; $J_{1^{\prime} \mathrm{b}-1^{\prime} \mathrm{a}}=15.2 \mathrm{~Hz} ; J_{1^{\prime} \mathrm{b}-2^{\prime}}=7.5 \mathrm{~Hz} ; J_{1^{\prime} \mathrm{b}-1}=$ $\left.6.7 \mathrm{~Hz} ; J_{1^{\prime} \mathrm{b}-3^{\prime}}=-1.6 \mathrm{~Hz} ; 1 \mathrm{H}\right) 2.34\left(\mathbf{H}-4^{\prime} \mathbf{b}\right.$; dddd; $J_{4^{\prime} \mathrm{b}-4^{\prime} \mathrm{a}}=$ $\left.15.2 \mathrm{~Hz} ; J_{4^{\prime} \mathrm{b}-3^{\prime}}=7.5 \mathrm{~Hz} ; J_{4^{\prime} \mathrm{b}-1}=6.7 \mathrm{~Hz} ; J_{4^{\prime} \mathrm{b}-2^{\prime}}=-1.6 \mathrm{~Hz} ; 1 \mathrm{H}\right)$ $2.09\left(\mathrm{CH}_{3} ; \mathrm{s} ; 6 \mathrm{H}\right) 1.23\left(\mathrm{H}-6 ; \mathrm{d} ; J_{6-5}=6.6 \mathrm{~Hz} ; 6 \mathrm{H}\right)$.

${ }^{13} \mathrm{C}$ NMR $\left(150 \mathrm{MHz}, \mathrm{CDCl}_{3}\right.$ ) $\delta$ ppm: $16.92(\mathrm{C}-6) 21.18$ $\left(\mathbf{C H}_{3}\right) 32.11\left(\mathbf{C}^{\prime} \mathbf{1}^{\prime}, \mathbf{C}-\mathbf{4}^{\prime}\right) 68.74(\mathbf{C}-4) 69.52(\mathbf{C}-5) 69.92(\mathbf{C}-1)$ $123.06(\mathbf{C}-3) 127.45\left(\mathbf{C}^{\prime} \mathbf{2}^{\prime}, \mathbf{C}^{\prime} \mathbf{3}^{\prime}\right) 133.39(\mathbf{C}-2) 170.69(\mathbf{C}=\mathbf{O})$.

HRMS $[\mathrm{M}+\mathrm{Na}]^{+}$. Experimental: 387.1760; calculated: 387.1778.

2.4.14. $7^{\prime}-O-\left(1^{\prime \prime \prime}-C-(4,6-D i-O-a c e t y l-2,3-d i d e o x y-\alpha-D\right.$-erythroheks-2-enpyranosyl)-but-2 $2^{\prime \prime \prime}$-en-4"'-yl)-genistein (3g)

E Isomer. ${ }^{1} \mathrm{H}$ NMR $\left(600 \mathrm{MHz}, \mathrm{CDCl}_{3}\right) \delta \mathrm{ppm:} 12.81\left(\mathbf{O H}-\mathbf{5}^{\prime}\right.$; s; $1 \mathrm{H}) 7.84\left(\mathbf{H}^{\prime}\right.$; s; $\left.1 \mathrm{H}\right) 7.36$ (H-2", $\mathbf{H}-6^{\prime \prime} ; \mathrm{d} ; J_{10^{\prime}-11^{\prime}, 14^{\prime}-13^{\prime}}=$ $8.63 \mathrm{~Hz} ; 2 \mathrm{H}) 6.87\left(\mathbf{H}^{\prime \prime} \mathbf{3}^{\prime \prime}, \mathbf{H}-5^{\prime \prime}\right.$; d; $\left.J_{11^{\prime}-10,13^{\prime}-14^{\prime}}=7.96 \mathrm{~Hz} ; 2 \mathrm{H}\right)$ 
$6.39\left(\mathbf{H}-\mathbf{8}^{\prime} ; \mathrm{d} ; J_{5^{\prime}-7^{\prime}}=2.65 \mathrm{~Hz} ; 1 \mathrm{H}\right) 6.38\left(\mathbf{H}-\mathbf{6}^{\prime} ; \mathrm{d} ; J_{7^{\prime}-5^{\prime}}=\right.$ 2.65 Hz; 1H) 5.96/5.79 (H-2, H-3, H-2'"', H-3'"'; m; 4H) 5.14 (H-4; broad signal; $1 \mathrm{H}) 4.55\left(\mathbf{H}-4^{\prime \prime \prime}\right.$; d; $\left.J_{4^{\prime \prime}-3^{\prime \prime}}=5.97 \mathrm{~Hz} ; 2 \mathrm{H}\right)$ $4.32\left(\mathrm{H}-1\right.$; ddd; $J_{1-1{ }^{\prime \prime}}=7.8 \mathrm{~Hz} ; J_{1-1{ }^{\prime \prime} \mathrm{b}}=5.47 \mathrm{~Hz} ; J_{1-2}=2.65 \mathrm{~Hz}$; $1 \mathrm{H}) 4.27\left(\mathbf{H}-6 \mathrm{a} ; \mathrm{dd} ; J_{6 \mathrm{a}-6 \mathrm{~b}}=11.94 \mathrm{~Hz} ; J_{6 \mathrm{a}-5}=6.63 \mathrm{~Hz} ; 1 \mathrm{H}\right) 4.16$ $\left(\mathbf{H}-6 \mathbf{b} ; \mathrm{dd} ; J_{6 \mathrm{~b}-6 \mathrm{a}}=11.61 \mathrm{~Hz} ; J_{6 \mathrm{~b}-5}=3.65 \mathrm{~Hz} ; 1 \mathrm{H}\right) 3.98(\mathbf{H}-5$; ddd; $\left.J_{5-6 \mathrm{a}}=6.3 \mathrm{~Hz} ; J_{5-4}=6.3 \mathrm{~Hz} ; J_{5-6 \mathrm{~b}}=3.98 \mathrm{~Hz} ; 1 \mathrm{H}\right) 2.52$ $\left(\mathrm{H}-1^{\prime \prime \prime} \mathrm{a}\right.$; ddd; $J_{1^{\prime \prime} \mathrm{a}-1^{\prime \prime} \mathrm{b}}=13.93 \mathrm{~Hz} ; J_{1^{\prime \prime} \mathrm{a}-1}=6.97 \mathrm{~Hz} ; J_{1^{\prime \prime} \mathrm{a}-2^{\prime \prime}}=$ $6.97 \mathrm{~Hz} ; 1 \mathrm{H}) 2.39\left(\mathbf{H}-\mathbf{1}^{\prime \prime \prime} \mathbf{b}\right.$; ddd; $J_{1^{\prime \prime} \mathrm{a}-1^{\prime \prime} \mathrm{b}}=14.60 \mathrm{~Hz} ; J_{1^{\prime \prime} \mathrm{b}-1}=$ $\left.5.97 \mathrm{~Hz} ; J_{1^{\prime \prime} \mathrm{b}-2^{\prime \prime}}=5.97 \mathrm{~Hz} ; 1 \mathrm{H}\right) 2.10(\mathrm{OAc} ; \mathrm{s} ; 6 \mathrm{H})$.

${ }^{13} \mathrm{C}$ NMR $\left(150 \mathrm{MHz}, \mathrm{CDCl}_{3}\right) \delta \mathrm{ppm}: 180.87\left(\mathrm{C}-4^{\prime}\right) 170.96$ $(\mathbf{C}=\mathbf{O}) 170.53(\mathbf{C}=\mathbf{O}) 164.44\left(\mathbf{C}-7^{\prime}\right) 162.57\left(\mathbf{C}-4^{\prime}\right) 157.88(\mathbf{C}-$ $\left.\mathbf{4}^{\prime \prime}\right) 156.18\left(\mathbf{C}^{\prime} \mathbf{8}^{\prime}\right.$ a) $152.76\left(\mathbf{C}-\mathbf{2}^{\prime}\right) 132.59$ (C-2) $131.07\left(\mathbf{C}-\mathbf{2}^{\prime \prime \prime}\right)$ $130.23\left(\mathbf{C}^{\prime \prime}{ }^{\prime \prime}, \mathbf{C}^{\prime \prime} \mathbf{6}^{\prime \prime}\right) 126.83\left(\mathbf{C}-\mathbf{3}^{\prime \prime \prime}\right) 123.90(\mathbf{C}-3) 123.68\left(\mathbf{C}^{\prime \prime} \mathbf{3}^{\prime}\right)$ $122.60\left(\mathbf{C}^{\prime \prime} \mathbf{1}^{\prime \prime}\right) 115.61\left(\mathbf{C}^{\prime} \mathbf{3}^{\prime \prime}, \mathbf{C}^{\prime} \mathbf{5}^{\prime \prime}\right) 106.23\left(\mathbf{C}-\mathbf{4}^{\prime} \mathbf{a}\right) 98.74\left(\mathbf{C}^{\prime} \mathbf{6}^{\prime}\right)$ $93.08\left(\mathbf{C}^{\prime} \mathbf{8}^{\prime}\right) 71.16(\mathbf{C}-1) 69.91(\mathbf{C}-5) 68.87\left(\mathbf{C}^{\prime \prime}{ }^{\prime \prime \prime}\right) 64.87$ (C-4) $62.77(\mathbf{C}-6) 36.60\left(\mathbf{C}-\mathbf{1}^{\prime \prime \prime}\right) 21.08\left(\mathbf{C H}_{3}{ }^{-}\right) 20.83\left(\mathbf{C H}_{3}{ }^{-}\right)$.

$Z$ Isomer. ${ }^{1} \mathrm{H}$ NMR $\left(600 \mathrm{MHz}, \mathrm{CDCl}_{3}\right) \delta$ ppm: $12.82\left(\mathbf{O H}^{\prime} \mathbf{5}^{\prime}\right.$; s; 1H) $7.85\left(\mathbf{H}^{\prime} \mathbf{2}^{\prime}\right.$; s; 1H) $7.36\left(\mathbf{H}^{\prime \prime} \mathbf{2}^{\prime \prime}, \mathbf{H}-\mathbf{6}^{\prime \prime}\right) 6.87\left(\mathbf{H}-\mathbf{3}^{\prime \prime}, \mathbf{H}-5^{\prime \prime}\right.$; $2 \mathrm{H}$; overlapped) $6.41\left(\mathbf{H}^{-8^{\prime}}\right.$; d; $\left.J_{5^{\prime}-7^{\prime}}=2.65 \mathrm{~Hz} ; 1 \mathrm{H}\right) 6.37\left(\mathbf{H}^{\prime} \mathbf{6}^{\prime}\right.$; d; $\left.J_{7^{\prime}-5^{\prime}}=2.65 \mathrm{~Hz} ; 1 \mathrm{H}\right) 5.96-5.79\left(\mathbf{H}-\mathbf{2}, \mathbf{H}-\mathbf{3}, \mathbf{H}-\mathbf{2}^{\prime \prime \prime}, \mathbf{H}-\mathbf{3}^{\prime \prime \prime}\right.$; m; $4 \mathrm{H}$; overlapped) 5.14 (H-4; broad signal; $1 \mathrm{H}$; overlapped) 4.66 $\left(\mathbf{H}-4^{\prime \prime \prime} ; \mathrm{d} ; J_{4^{\prime \prime}-3^{\prime \prime}}=5.31 \mathrm{~Hz} ; 2 \mathrm{H}\right) 4.32(\mathbf{H}-\mathbf{1} ; 1 \mathrm{H}$; overlapped $)$ $4.29\left(\mathbf{H}-6 \mathrm{a} ; \mathrm{d} ; J_{6 \mathrm{a}-6 \mathrm{~b}}=11.94 \mathrm{~Hz} ; 1 \mathrm{H} ;\right.$ partially overlapped) 4.16 $\left(\mathbf{H}-6 \mathbf{b} ; 1 \mathrm{H}\right.$; overlapped) $4.01\left(\mathbf{H}-5\right.$; ddd; $J_{5-6 a}=6.47 \mathrm{~Hz} ; J_{5-4}=$ $\left.6.47 \mathrm{~Hz} ; J_{5-6 \mathrm{~b}}=3.65 \mathrm{~Hz} ; 1 \mathrm{H}\right) 2.52\left(\mathbf{H}-\mathbf{1}^{\prime \prime \prime} \mathbf{a} ; 1 \mathrm{H}\right.$; overlapped $)$ $2.46\left(\mathbf{H}-\mathbf{1}^{\prime \prime \prime} \mathrm{b} ; \mathrm{ddd} ; J_{1^{\prime \prime} \mathrm{b}-1^{\prime \prime} \mathrm{a}}=14.60 \mathrm{~Hz} ; J_{1^{\prime \prime} \mathrm{b}-1}=5.97 \mathrm{~Hz} ; J_{1^{\prime \prime} \mathrm{b}-2^{\prime \prime}}\right.$ $=5.97 \mathrm{~Hz} ; 1 \mathrm{H}) 2.10(\mathbf{O A c} ; \mathrm{s} ; 6 \mathrm{H})$.

${ }^{13} \mathrm{C} \mathrm{NMR}\left(150 \mathrm{MHz}, \mathrm{CDCl}_{3}\right) \delta$ ppm: $180.87\left(\mathrm{C}-4^{\prime}\right.$; overlapped) $170.96(\mathbf{C}=\mathbf{O}$; overlapped $) 170.53(\mathbf{C}=\mathbf{O}$; overlapped $)$ 164.44 (C-7'; overlapped) 162.57 (C-5' ${ }^{\prime}$; overlapped) 157.88 (C-4' ${ }^{\prime \prime}$; overlapped) $156.18\left(\mathbf{C}-8^{\prime} \mathbf{a}\right.$; overlapped) $152.76\left(\mathbf{C - 2}^{\prime}\right.$; overlapped) 132.50 (C-2) 130.23 (C-2", C-6"'; overlapped) $129.88\left(\mathbf{C - 2}^{\prime \prime \prime}\right) 126.30\left(\mathbf{C}^{\prime \prime} \mathbf{3}^{\prime \prime \prime}\right) 124.02$ (C-3) 123.68 (C-3'; overlapped) 122.60 (C-4' $\mathbf{a}$; overlapped) $115.61\left(\mathbf{C}-\mathbf{3}^{\prime \prime}, \mathbf{C}-\mathbf{5}^{\prime \prime}\right.$; overlapped) 106.23 (C-3'; overlapped) $98.68\left(\mathbf{C}^{\prime} \mathbf{6}^{\prime}\right) 93.11$ $\left(\mathbf{C}^{\prime} \mathbf{8}^{\prime}\right) 70.93$ (C-1) 70.15 (C-5) 64.55 (C-4"') 64.87 (C-4; overlapped) $62.77(\mathbf{C}-6$; overlapped $) 32.11\left(\mathbf{C}-\mathbf{1}^{\prime \prime \prime}\right) 21.08\left(\mathbf{C H}_{3}\right.$; overlapped) $20.83\left(\mathbf{C H}_{3}\right.$; overlapped $)$.

HRMS $[\mathrm{M}+\mathrm{Na}]^{+}$. Experimental: 559.1560; calculated: 559.1575 .

\subsubsection{5. $1^{\prime}, 4^{\prime}$-Bis-C-(4,6-di-O-acetyl-2,3-dideoxy- $\alpha$-D-erythro- heks-2-enpyranosyl)-but-2'-en (4g)}

E Isomer. ${ }^{1} \mathrm{H}$ NMR $\left(300 \mathrm{MHz}, \mathrm{CDCl}_{3}\right) \delta$ ppm: $5.93(\mathbf{H}-2$; ddd; $\left.J_{2-3}=10.45 \mathrm{~Hz} ; J_{2-1}=2.41 \mathrm{~Hz} ; J_{2-4}=1.51 \mathrm{~Hz} ; 2 \mathrm{H}\right) 5.80$ $\left(\mathbf{H}-3\right.$; ddd; $J_{3-2}=10.30 \mathrm{~Hz} ; J_{3-4}=2.83 \mathrm{~Hz} ; J_{2-1}=2.08 \mathrm{~Hz}$; 2H) $5.58\left(\mathbf{H}^{\prime} \mathbf{2}^{\prime}, \mathbf{H}-\mathbf{3}^{\prime}\right.$; t $; 3.7 \mathrm{~Hz} \times 2$; $\left.2 \mathrm{H}\right) 5.14(\mathbf{H}-\mathbf{4} ; \mathrm{m} ; 2 \mathrm{H})$ $4.26\left(\mathbf{H}-6 \mathbf{a} ; \mathrm{dd} ; J_{6 \mathrm{a}-6 \mathrm{~b}}=11.92 \mathrm{~Hz} ; J_{6 \mathrm{a}-5}=6.32 \mathrm{~Hz} ; 2 \mathrm{H}\right) 4.26$ (H-1; overlapped przez H-6a; 2H) 4.14 (H-6b; ddd; $J_{6 \mathrm{~b}-6 \mathrm{a}}=$ $\left.12.06 \mathrm{~Hz} ; J_{6 \mathrm{~b}-5}=3.59 \mathrm{~Hz} ; 2 \mathrm{H}\right) 3.95\left(\mathbf{H}-5\right.$; ddd; $J_{5-6 \mathrm{a}}=6.43 \mathrm{~Hz}$; $\left.J_{5-4}=6.43 ; J_{5-6 b}=3.38 \mathrm{~Hz} ; 2 \mathrm{H}\right) 2.49 / 2.25\left(\mathbf{H - 1}^{\prime} \mathbf{a}, \mathbf{H}-\mathbf{l}^{\prime} \mathbf{b}, \mathbf{H}-\right.$ $\left.\mathbf{4}^{\prime} \mathbf{a}, \mathbf{H}-\mathbf{4}^{\prime} \mathbf{b} ; \mathrm{m} ; 4 \mathrm{H}\right) 2.10\left(-\mathrm{CH}_{3} ; \mathrm{s} ; 12 \mathrm{H}\right)$.

${ }^{13} \mathrm{C}$ NMR $\left(75 \mathrm{MHz}, \mathrm{CDCl}_{3}\right) \delta$ ppm: $170.83(\mathrm{C}=\mathbf{O}) 170.42$ $(\mathbf{C}=\mathbf{O}) 132.85(\mathbf{C}-2) 128.61\left(\mathbf{C}^{\prime} \mathbf{2}^{\prime}, \mathbf{C}^{\prime} \mathbf{3}^{\prime}\right) 123.65(\mathbf{C}-3) 71.64$
(C-5) 69.83 (C-1) 64.92 (C-4) 62.79 (C-6) $36.77\left(\right.$ C-1' $\left.^{\prime}, C^{\prime}-4^{\prime}\right)$ $21.10\left(\mathbf{C H}_{3}\right) 20.85\left(\mathbf{C H}_{3}\right)$.

$Z$ Isomer. ${ }^{1} \mathrm{H} \mathrm{NMR}\left(300 \mathrm{MHz}, \mathrm{CDCl}_{3}\right) \delta$ ppm: 5.94 (H-2; ddd; $\left.J_{2-3}=10.45 \mathrm{~Hz} ; J_{2-1}=2.41 \mathrm{~Hz} ; J_{2-4}=1.36 \mathrm{~Hz} ; 2 \mathrm{H}\right) 5.82(\mathbf{H}-3$; ddd; $\left.J_{3-2}=10.30 \mathrm{~Hz} ; J_{3-4}=2.84 \mathrm{~Hz} ; J_{2-1}=2.08 \mathrm{~Hz} ; 2 \mathrm{H}\right) 5.61$ $\left(\mathbf{H}^{\prime}{ }^{\prime}, \mathbf{H}-\mathbf{3}^{\prime} ; \mathrm{t} ; 4.9 \mathrm{~Hz} \times 2 ; 2 \mathrm{H}\right) 5.14(\mathbf{H}-4 ; \mathrm{m} ; 2 \mathrm{H}) 4.26(\mathbf{H}-$ 6a; overlapped; $2 \mathrm{H}) 4.26(\mathbf{H}-1$; overlapped; $2 \mathrm{H}) 4.14(\mathbf{H}-6 \mathbf{b}$; overlapped; $2 \mathrm{H}) 3.96\left(\mathrm{H}-5\right.$; ddd; $J_{5-6 \mathrm{a}}=6.57 \mathrm{~Hz} ; J_{5-4}=6.57 \mathrm{~Hz}$; $\left.J_{5-6 \mathrm{~b}}=3.23 \mathrm{~Hz} ; 2 \mathrm{H}\right) 2.49-2.25\left(\mathbf{H}^{\prime} \mathbf{1}^{\prime} \mathbf{a}, \mathbf{H}^{\prime} \mathbf{1}^{\prime} \mathbf{b}, \mathbf{H}-\mathbf{4}^{\prime} \mathbf{a}, \mathbf{H}-\mathbf{4}^{\prime} \mathbf{b} ; \mathbf{m}\right.$; 4H) $2.09\left(-\mathrm{CH}_{3}\right.$; ; 12H).

${ }^{13} \mathrm{C}$ NMR $\left(75 \mathrm{MHz}, \mathrm{CDCl}_{3}\right) \delta \mathrm{ppm}: 170.83(\mathbf{C}=\mathbf{O}$; overlapped) $170.42(\mathbf{C}=\mathbf{O}$; overlapped) 132.77 (C-2) 127.30 (C$\left.\mathbf{2}^{\prime}, \mathbf{C}^{\prime} \mathbf{3}^{\prime}\right) 123.77$ (C-3) 71.39 (C-5) 69.98 (C-1) 64.92 (C-4; overlapped) 62.79 (C-6; overlapped) $31.71\left(\mathbf{C}^{\prime} \mathbf{1}^{\prime}, \mathbf{C}-\mathbf{4}^{\prime}\right)$ 21.1020.85 ( $\mathbf{C H}_{3}$; overlapped).

HRMS $[\mathrm{M}+\mathrm{Na}]^{+}$. Experimental: 503.1906; calculated: 503.1888 .

2.4.16. $\quad 7^{\prime}-O-\left(I^{\prime \prime \prime}-C-(4,6-D i-O-a c e t y l-2,3-d i d e o x y-\alpha-D\right.$-treoheks-2-enpyranosyl)-but-2"' -en-4"' -yl)-genistein (3h)

Isomer E. ${ }^{1} \mathrm{H}$ NMR $\left(300 \mathrm{MHz}, \mathrm{CDCl}_{3}\right) \delta \mathrm{ppm}: 12.81\left(\mathbf{H}-\mathbf{5}^{\prime}\right.$; s; 1H) $7.83\left(\mathbf{H}^{\prime}\right.$; s; $\left.1 \mathrm{H}\right) 7.35 / 7.33\left(\mathbf{H}^{\prime \prime} \mathbf{2}^{\prime \prime}, \mathbf{H}-\mathbf{6}^{\prime \prime} ; \mathrm{m} ; 2 \mathrm{H}\right)$ $6.87 / 6.84\left(\mathbf{H}^{\prime \prime} \mathbf{3}^{\prime \prime} ; \mathbf{H}-\mathbf{5}^{\prime \prime} ; \mathrm{m} ; 2 \mathrm{H}\right) 6.39\left(\mathbf{H}-\mathbf{8}^{\prime} ; \mathrm{d} ; J_{5^{\prime}-7^{\prime}}=2.3 \mathrm{~Hz}\right.$; 1H) $6.35\left(\mathbf{H}-\mathbf{6}^{\prime}\right.$; d; $\left.J_{7^{\prime}-5^{\prime}}=2.3 \mathrm{~Hz} ; 1 \mathrm{H}\right) 6.07-5.98(\mathbf{H}-\mathbf{2}, \mathbf{H}-3$; m; $2 \mathrm{H}) 5.91\left(\mathbf{H}-2^{\prime \prime \prime}\right.$; ddd; $J_{2^{\prime \prime}-3^{\prime \prime}}=15.6 \mathrm{~Hz} ; J_{2^{\prime \prime}-1^{\prime \prime} \mathrm{a}}=6.5 \mathrm{~Hz} ; J_{2^{\prime \prime}-11^{\prime \prime} \mathrm{b}}$ $=6.4 \mathrm{~Hz} ; 1 \mathrm{H}) 5.80\left({\mathrm{H}-3^{\prime \prime \prime}}^{\prime \prime} ; \mathrm{dt} ; J_{3^{\prime \prime}-2^{\prime \prime}}=15.6 \mathrm{~Hz} ; J_{3^{\prime \prime}-4^{\prime \prime}}=5.3 \mathrm{~Hz}\right.$ $\times 2 ; 1 \mathrm{H}) 5.09\left(\mathbf{H}-4\right.$; dd; $\left.J_{4-3}=4.3 \mathrm{~Hz} ; J_{4-5}=2.7 \mathrm{~Hz} ; 1 \mathrm{H}\right) 4.54(\mathbf{H}-$ $4^{\prime \prime \prime}$; d; $\left.J_{4^{\prime \prime}-3^{\prime \prime}}=5.3 \mathrm{~Hz} ; 1 \mathrm{H}\right) 4.42-4.37(\mathbf{H}-\mathbf{1} ; \mathrm{m} ; 1 \mathrm{H}) 4.24 / 4.21$ $\left(\mathbf{H}-6 \mathbf{a}, \mathrm{Hz} ; J_{1^{\prime \prime} \mathrm{a}-1}=8.3 \mathrm{~Hz} ; 1 \mathrm{H}\right) 2.35\left(\mathbf{H}-\mathbf{1}^{\prime \prime \prime} \mathbf{b}\right.$; ddd; $J_{1^{\prime \prime} \mathrm{b}-1^{\prime \prime} \mathrm{a}}=$ $\left.14.6 \mathrm{~Hz} ; J_{1^{\prime \prime} \mathrm{b}-2^{\prime \prime}}=6.4 \mathrm{~Hz} ; J_{1^{\prime \prime} \mathrm{b}-1}=5.7 \mathrm{~Hz} ; 1 \mathrm{H}\right) 2.09\left(-\mathbf{C H}_{3} ; \mathrm{s}\right.$; 3H) $2.09\left(-\mathrm{CH}_{3} ; \mathrm{s} ; 3 \mathrm{H}\right)$.

${ }^{13} \mathrm{C}$ NMR $\left(75 \mathrm{MHz}, \mathrm{CDCl}_{3}\right) \delta \mathrm{ppm}: 20.79\left(-\mathrm{CH}_{3}\right) 20.87$ $\left(-\mathrm{CH}_{3}\right) 35.22\left(\mathbf{C}^{\prime \prime \prime}{ }^{\prime \prime \prime}\right) 62.89$ (C-6) 63.80 (C-4) 68.07 (C-5) $68.80\left(\mathbf{C}^{\prime \prime \prime \prime}\right) 72.11(\mathbf{C}-1) 93.03\left(\mathbf{C}^{\prime \prime} \mathbf{8}^{\prime}\right) 98.74\left(\mathbf{C}^{\prime} \mathbf{6}^{\prime}\right) 106.19$ (C$\left.4^{\prime} \mathbf{a}\right) 115.62\left(\mathbf{C}-3^{\prime \prime}, \mathbf{C}-5^{\prime \prime}\right) 122.27(\mathbf{C}-3) 122.49\left(\mathbf{C}-\mathbf{1}^{\prime \prime}\right) 123.67(\mathbf{C}-$ $\left.3^{\prime}\right) 126.94\left(\mathbf{C}-3^{\prime \prime \prime}\right) 130.18\left(\mathbf{C}-2^{\prime \prime}, \mathbf{C}-6^{\prime \prime}\right) 130.84\left(\mathbf{C}-2^{\prime \prime \prime}\right) 134.37$ (C-2) $152.79\left(\mathbf{C}^{\prime} \mathbf{2}^{\prime}\right) 156.34\left(\mathbf{C}-5^{\prime}\right) 157.82\left(\mathbf{C}-8^{\prime} \mathbf{a}\right) 162.47\left(\mathbf{C}-4^{\prime \prime}\right)$ $164.42\left(\mathbf{C}-7^{\prime}\right) 170.70(\mathbf{C}=\mathbf{O}) 170.98(\mathbf{C}=\mathbf{O}) 180.88\left(\mathbf{C}-\mathbf{4}^{\prime}\right)$.

$Z$ Isomer. ${ }^{1} \mathrm{H} \mathrm{NMR}\left(300 \mathrm{MHz}, \mathrm{CDCl}_{3}\right) \delta \mathrm{ppm}: 12.81\left(\mathbf{H}-\mathbf{5}^{\prime}\right.$; s; 1H) $7.85\left(\mathbf{H}-\mathbf{2}^{\prime}\right.$; s; $\left.1 \mathrm{H}\right) 7.35 / 7.33\left(\mathbf{H}-\mathbf{2}^{\prime \prime}, \mathbf{H}-\mathbf{6}^{\prime \prime}\right.$; overlapped; $\left.2 \mathrm{H}\right)$ $6.87 / 6.84\left(\mathbf{H}^{\prime \prime}{ }^{\prime \prime}\right.$; H-5 $\mathbf{5}^{\prime \prime}$; overlapped; $\left.2 \mathrm{H}\right) 6.40\left(\mathbf{H}-\mathbf{8}^{\prime}\right.$; d; $J_{5^{\prime}-7^{\prime}}$ $=2.3 \mathrm{~Hz} ; 1 \mathrm{H}) 6.36\left(\mathbf{H}^{-} \mathbf{6}^{\prime}\right.$; d; $\left.J_{7^{\prime}-5^{\prime}}=2.3 \mathrm{~Hz} ; 1 \mathrm{H}\right) 6.07 / 5.98(\mathbf{H}-$ 2, H-3; overlapped; $2 \mathrm{H}) 5.85 / 5.76\left(\mathbf{H}-\mathbf{2}^{\prime \prime \prime}, \mathbf{H}-\mathbf{3}^{\prime \prime \prime}\right.$; overlapped; 1H) $5.12\left(\mathrm{H}-4 ; \mathrm{dd} ; J_{4-3}=4.0 \mathrm{~Hz} ; J_{4-5}=2.2 \mathrm{~Hz} ; 1 \mathrm{H}\right) 4.65$ $\left(\mathbf{H}-4^{\prime \prime \prime} ; \mathrm{d} ; J_{4^{\prime \prime}-3^{\prime \prime}}=4.6 \mathrm{~Hz} ; 1 \mathrm{H}\right) 4.42 / 4.37$ (H-1; overlapped; 1H) 4.24/4.21 (H-6a, H-6b; overlapped; $2 \mathrm{H})$ 4.18/4.13 (H-5; overlapped; $1 \mathrm{H})$ 2.57/2.43 (H-1'"' a, H-1'"'b; overlapped; $2 \mathrm{H})$ $2.10\left(-\mathrm{CH}_{3} ; \mathrm{s} ; 3 \mathrm{H}\right) 2.08\left(-\mathrm{CH}_{3} ; \mathrm{s} ; 3 \mathrm{H}\right)$.

${ }^{13} \mathrm{C}$ NMR $\left(75 \mathrm{MHz}, \mathrm{CDCl}_{3}\right) \delta \mathrm{ppm}: 20.79\left(-\mathrm{CH}_{3}\right.$, overlapped) $20.87\left(-\mathbf{C H}_{3}\right.$, overlapped) $31.04\left(\mathbf{C}-\mathbf{1}^{\prime \prime \prime}\right) 62.81(\mathbf{C}$ 6) $63.80(\mathbf{C}-4) 64.43\left(\mathbf{C}-4^{\prime \prime \prime}\right) 68.18$ (C-5, overlapped) 71.92 (C-1) $93.03\left(\mathbf{C}^{\prime} \mathbf{8}^{\prime}\right.$, overlapped) $98.68\left(\mathbf{C}^{-6^{\prime}}\right) 106.24\left(\mathbf{C}-\mathbf{4}^{\prime} \mathbf{a}\right)$ 115.62 (C-3", C-5", overlapped) 122.39 (C-3) 122.49 (C-1", overlapped) $123.67\left(\mathbf{C}-3^{\prime}\right.$, overlapped) $126.24\left(\mathbf{C}-3^{\prime \prime \prime}\right) 129.87$ 
$\left(\mathbf{C}^{\prime \prime} \mathbf{2}^{\prime \prime}\right) 130.18$ (C-2 ${ }^{\prime \prime}, \mathbf{C}^{\prime \prime}{ }^{\prime \prime}$, overlapped) 134.16 (C-2) 152.79 (C-2', overlapped) 156.34 (C-5', overlapped) $157.82\left(\mathbf{C}-8^{\prime} \mathbf{a}\right.$, overlapped) $162.517\left(\mathbf{C}-4^{\prime \prime}\right) 164.39\left(\mathbf{C}^{\prime} \mathbf{6}^{\prime}\right) 170.70(\mathbf{C}=\mathbf{O}$, overlapped) $170.98\left(\mathbf{C}=\mathbf{O}\right.$, overlapped) $180.88\left(\mathbf{C}-4^{\prime}\right.$, overlapped $)$.

HRMS $[\mathrm{M}+\mathrm{Na}]^{+}$. Experimental: 559.1552; calculated: 559.1575.

\subsubsection{7. $1^{\prime}, 4^{\prime}$-Bis-C-(4,6-di-O-acetyl-2,3-dideoxy- $\alpha$-D-treo- heks-2-enpyranosyl)-but-2' -en (4h)}

E Isomer. ${ }^{1} \mathrm{H}$ NMR $\left(300 \mathrm{MHz}, \mathrm{CDCl}_{3}\right) \delta$ ppm: $6.04(\mathbf{H}-2 \mathrm{dd}$; $\left.J_{2-3}=10.2 \mathrm{~Hz} ; J_{2-1}=2.7 \mathrm{~Hz} ; 2 \mathrm{H}\right) 5.98\left(\mathbf{H}-3\right.$; ddd; $J_{3-2}=10.2 \mathrm{~Hz}$; $\left.J_{3-4}=4.7 \mathrm{~Hz} ; J_{3-1}=1.7 \mathrm{~Hz} ; 2 \mathrm{H}\right) 5.59\left(\mathbf{H}^{\prime} \mathbf{2}^{\prime}, \mathbf{H}-\mathbf{3}^{\prime}\right.$; t; $3.8 \mathrm{~Hz} \times 2$; $2 \mathrm{H}) 5.08$ (H-4; dd; $\left.J_{4-3}=4.7 \mathrm{~Hz} ; J_{4-5}=2.3 \mathrm{~Hz} ; 2 \mathrm{H}\right) 4.32(\mathbf{H}-\mathbf{1}$; dddd; $J_{1-1^{\prime} \mathrm{a}}=7.8 ; J_{1-1^{\prime} \mathrm{b}}=5.2 \mathrm{~Hz} ; J_{1-2}=2.7 \mathrm{~Hz} ; J_{1-3}=1.7 \mathrm{~Hz}$; 2H) 4.21/4.19 (H-6a, H-6b; m; 4H) 4.16-4.10 (H-5; m; 2H) 2.46-2.35 (H-1' a, H-4' a; m; 2H) 2.31/2.23 (H-1' b, H-4' b; m; 2H) $2.09\left(-\mathrm{CH}_{3} ; \mathrm{s} ; 6 \mathrm{H}\right) 2.08\left(-\mathrm{CH}_{3} ; \mathrm{s} ; 6 \mathrm{H}\right)$.

${ }^{13} \mathrm{C}$ NMR $\left(75 \mathrm{MHz}, \mathrm{CDCl}_{3}\right) \delta \mathrm{ppm}: 20.81\left(-\mathrm{CH}_{3}\right) 20.90$ $\left(-\mathrm{CH}_{3}\right) 35.72\left(\mathrm{C}-1^{\prime}, \mathrm{C}^{\prime} \mathbf{4}^{\prime}\right) 62.86(\mathrm{C}-6) 63.77$ (C-4) $68.04(\mathrm{C}-$ 5) 72.58 (C-1) $122.04(\mathbf{C}-3) 128.53\left(\mathbf{C}^{\prime}{ }^{\prime}, \mathbf{C}^{\prime} \mathbf{3}^{\prime}\right) 134.69$ (C-2) $170.52(\mathbf{C}=\mathbf{O}) 170.70(\mathbf{C}=\mathbf{O})$.

$Z$ Isomer. ${ }^{1} \mathrm{H}$ NMR $\left(300 \mathrm{MHz}, \mathrm{CDCl}_{3}\right) \delta$ ppm: 6.06 (H-2; dd; $\left.J_{2-3}=10.4 \mathrm{~Hz} ; J_{2-1}=2.9 \mathrm{~Hz} ; 2 \mathrm{H}\right) 5.98(\mathbf{H}-3$; overlapped; $2 \mathrm{H})$ $5.63\left(\mathbf{H}^{\prime}{ }^{\prime}\right.$; t $\left.; 4.8 \mathrm{~Hz} \times 2 ; 2 \mathrm{H}\right) 5.08(\mathbf{H}-4$; overlapped; $2 \mathrm{H}) 4.32$ (H-1; overlapped; 2H) 4.21/4.19 (H-6a, H-6b; overlapped; $4 \mathrm{H})$ 4.16/4.10 (H-5; overlapped; $2 \mathrm{H})$ 2.46/2.35 (H-1' $\mathbf{a}, \mathbf{H}-\mathbf{1}^{\prime} \mathbf{b}$, $\mathbf{H}^{-} \mathbf{4}^{\prime} \mathbf{a}, \mathbf{H}-4^{\prime} \mathbf{b}$; overlapped; $\left.4 \mathrm{H}\right)$ 2.09/2.08 (- $\mathbf{C H}_{3}$; overlapped; $12 \mathrm{H})$.

${ }^{13} \mathrm{C} \mathrm{NMR}\left(75 \mathrm{MHz}, \mathrm{CDCl}_{3}\right) \delta \mathrm{ppm}: 20.81\left(-\mathbf{C H}_{3}\right.$, overlapped) $20.90\left(-\mathbf{C H}_{3}\right.$, overlapped) $30.66\left(\mathbf{C - 1}{ }^{\prime}, \mathbf{C}-4^{\prime}\right) 62.86$ (C-6, overlapped) 63.74 (C-4) 68.09 (C-5) 72.32 (C-1) 122.16

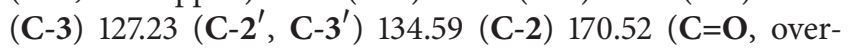
lapped) $170.70(\mathbf{C}=\mathbf{O}$, overlapped $)$.

HRMS $[\mathrm{M}+\mathrm{Na}]^{+}$. Experimental: 503.1911; calculated: 503.1888 .

2.5. Cancer Cell Lines. HCT 116 colorectal cancer and DU 145 prostate cancer cell lines obtained from ATCC (American Type Culture Collection, Rockville, MD, USA) were cultured in RPMI 1640 medium supplemented with $10 \%$ of fetal bovine serum (FBS) (MP Biomedicals) and $1 \mu \mathrm{g} / \mathrm{mL}$ gentamicin (KRKA, Novo Mesto, Slovenia), at $37^{\circ} \mathrm{C}$ in a humidified atmosphere containing $5 \% \mathrm{CO}_{2}$ in the air. Cells were split at $90 \%$ confluence. The grown cells were detached by rinsing with $0.02 \%$ ethylenediamine tetraacetic acid (EDTA) followed by $0.25 \%$ trypsin.

2.6. Cytotoxicity Assays. Cell viability was estimated using an MTT (3-[4,5-dimethylthiazol-2-y]2,5-diphenyltetrazolium bromide) assay (Sigma-Aldrich, Germany), according to the supplier's protocol. After $72 \mathrm{~h}$ treatment with tested agents (3a and $3 \mathbf{f}$ ) at concentration series: $0.1,1,5,10,25$, and $50 \mu \mathrm{M}$ the medium was aspirated, and cells were incubated for 3 hours at $37^{\circ} \mathrm{C}$ with $0.5 \mathrm{mg} / \mathrm{mL}$ MTT solution $(50 \mu \mathrm{L})$ in Dulbecco modified essential medium (DMEM) (Sigma-Aldrich,
Germany) without phenol red. Then the medium was aspired, insoluble crystals of formazan were solubilized in 2propanol: $\mathrm{HCl}$ solution, and optical density $(\lambda=570 \mathrm{~nm})$ was determined in a microplate reader BioTek Synergy II (BioTek Instruments, USA). $\mathrm{IC}_{50}$ was estimated using CalcuSyn software using the 4 parameter nonlinear regression model for curve-fitting analysis. The experiments were repeated at least three times.

2.7. Cell Cycle Analysis and Microscopy Studies. Cell cycle analysis was performed after $24 \mathrm{~h}$ treatment of cells with the tested compounds with FACSCanto flow cytometer as described elsewhere [17]. Microscope analysis of cell morphology was performed with use of inverted OLYMPUS IX 70 microscope (Olympus, Japan) equipped with differential interference contrast. Confocal images of cells treated with the $\mathbf{3} \mathbf{a}$ and $\mathbf{3 f}$ derivatives were taken with Zeiss LSM 710 (Carl Zeiss, Germany) with use of $63 x$ oil objective. For nuclei visualization cells were treated with $5 \mu \mathrm{g} / \mathrm{mL}$ Hoechst 33342 (Sigma-Aldrich, Germany) for 90 minutes, and for lysosomes and mitochondria staining cells were treated with $100 \mathrm{nM}$ Lysotracker red and $200 \mathrm{nM}$ Mitotracker green (both from Molecular Probes, USA) for 30 minutes.

2.8. Determination of Compounds Stability. $50 \% \mathrm{HCO}_{2} \mathrm{H}$ in $\mathrm{H}_{2} \mathrm{O}$ for HPLC were supplied by Sigma-Aldrich, Germany, and $\mathrm{MeCN}$ and $\mathrm{H}_{2} \mathrm{O}$ for LC-MS were supplied by $\mathrm{POCH}$, Poland. For analytes preparation HCT 116 cells were plated in 24-well plate (at 25\% confluence). Cells were cultured at $37^{\circ} \mathrm{C}$ in humidified atmosphere of $5 \% \mathrm{CO}_{2}$ for $48 \mathrm{~h}$. The culture media consisted of RPMI, $10 \%$ fetal bovine serum, and $0.1 \%$ gentamycin sulfate. After $48 \mathrm{~h}$ the culture medium was removed from wells and replaced by $1 \mathrm{~mL} 10 \mu \mathrm{M}$ solution of $\mathbf{3} \mathbf{a}$ and $\mathbf{3} \mathbf{f}$ in a fresh medium. Cells were incubated for additional $8 \mathrm{~h}$ or $24 \mathrm{~h}$. Then the medium was collected and centrifuged $(2000 \mathrm{~g}, 2 \mathrm{~min})$. The supernatant was filtrated through a syringe filter $(0.22 \mu \mathrm{L}, \mathrm{RC})$ and transferred to an autosampler vial. The supernatant was analyzed by LCMS/MS. In control experiments, compounds were incubated with medium without cells (RPMI supplemented with $10 \%$ fetal bovine serum and $0.1 \%$ gentamycin sulfate). The LC separation was performed using a Dionex UHPLC system (Dionex Corporation, Sunnyvalem, CA, USA) consisting of an UltiMate 3000 RS pump, an UltiMate 3000 autosampler, an UltiMate 3000 column compartment, and an UltiMate 3000 variable wavelength detector. UHPLC system was operated using Dionex Chromaleon 6.8 software. Chromatography was performed using a C18 ACE column $(150 \times 4.6 \mathrm{~mm}$, $3.0 \mu \mathrm{m}$, Advance Chromatogr. Technologies, Aberdeen, UK) connected by integral holder $(3.2 \times 4.6 \mathrm{~mm})$ with guard column of the same material. Isocratic conditions were applied: $25 \%$ of $0.1 \%$ aq. $\mathrm{HCO}_{2} \mathrm{H}$ and $75 \%$ of $\mathrm{MeCN}$, the flow rate was set at $0.3 \mathrm{~mL} / \mathrm{min}$, and sample injection volume was $5 \mu \mathrm{L}$. The HPLC column was thermostated at $25^{\circ} \mathrm{C}$, and samples were kept in the autosampler at $10^{\circ} \mathrm{C}$. Compounds were detected at $\lambda 260 \mathrm{~nm}$. In order to determine the mass of compounds, the UHPLC system was connected to a $4000 \mathrm{Q}$ TRAP triple quadrupole linear ion trap mass spectrometer 
TABLE 1: CM reactions of C-allyl glycosides (1a-1h) and exo-glycal (1i) with 7-O-allylgenistein (2).

\begin{tabular}{|c|c|c|c|c|}
\hline Entry & Sugar & $\begin{array}{l}\text { Glycoconjugate } \\
\text { Yields }^{\mathrm{a}} \\
(\mathrm{E} / \mathrm{Z})^{\mathrm{c}}\end{array}$ & $\begin{array}{l}\text { C-Allyl glycoside homodimer } \\
\text { Yields }(\%)^{\mathrm{a}} \\
(\mathrm{E} / \mathrm{Z})^{\mathrm{c}}\end{array}$ & $\begin{array}{l}\text { 7-O-Allylgenistein } \\
\text { homodimer } \\
\text { Yields }(\%)^{\mathrm{b}} \\
(\mathrm{E} / \mathrm{Z})^{\mathrm{c}} \\
\end{array}$ \\
\hline 1 & la & $\begin{array}{c}\mathbf{3 a} \\
42 \%(2.5) \\
25 \%(10)^{\mathrm{d}}\end{array}$ & $\begin{array}{c}\mathbf{4 a} \\
7 \%(2.5) \\
18 \%(\text { only E) } \\
\text { d }\end{array}$ & $\begin{array}{c}\mathbf{5} \\
57 \%(2.0) \\
47 \%(11.4)^{\mathrm{d}}\end{array}$ \\
\hline 2 & $1 b$ & $\begin{array}{c}3 \mathbf{b} \\
41 \%(2.1)\end{array}$ & $\begin{array}{c}\mathbf{4 b} \\
29 \%(1.8)\end{array}$ & $\begin{array}{c}\mathbf{5} \\
36 \%(2.0)\end{array}$ \\
\hline 3 & lc & $\begin{array}{c}3 \mathbf{c} \\
44 \%(2.2)\end{array}$ & $\begin{array}{c}4 \mathbf{c} \\
30 \% \text { (n.d.) }\end{array}$ & $\begin{array}{c}\mathbf{5} \\
43 \%(2.0)\end{array}$ \\
\hline 4 & 1d & $\begin{array}{c}\mathbf{3 d} \\
18 \%(2.6)\end{array}$ & $\begin{array}{c}\mathbf{4 d} \\
6 \%(2.0)\end{array}$ & $\begin{array}{c}\mathbf{5} \\
30 \%(2.0)\end{array}$ \\
\hline 5 & le & $\begin{array}{c}\mathbf{3 e} \\
33 \%(2.1)\end{array}$ & $\begin{array}{c}\mathbf{4 e} \\
9 \%(1.5)\end{array}$ & $\begin{array}{c}\mathbf{5} \\
47 \%(2.0)\end{array}$ \\
\hline 6 & If & $\begin{array}{c}\mathbf{3 f} \\
20 \%(2.2)\end{array}$ & $\begin{array}{c}\mathbf{4 f} \\
8 \%(1.6)\end{array}$ & $\begin{array}{c}\mathbf{5} \\
13 \%(2.0)\end{array}$ \\
\hline 7 & $1 g$ & $\begin{array}{c}\mathbf{3 g} \\
4 \%(2.4)\end{array}$ & $\begin{array}{c}\mathbf{4 g} \\
13 \%(1.5)\end{array}$ & $\begin{array}{c}\mathbf{5} \\
28 \%(2.0)\end{array}$ \\
\hline 8 & lh & $\begin{array}{c}\mathbf{3 h} \\
35 \%(2.8) \\
17 \%(7.3)^{\mathrm{d}}\end{array}$ & $\begin{array}{c}\mathbf{4 h} \\
26 \%(2.4) \\
5 \%(\text { only E) } \\
\text { d }\end{array}$ & $\begin{array}{c}\quad 5 \\
29 \%(2.0) \\
18 \%(11.4)^{\mathrm{d}}\end{array}$ \\
\hline 9 & li & - & - & $\begin{array}{c}\mathbf{5} \\
21 \%(2.0)\end{array}$ \\
\hline
\end{tabular}

${ }^{a}$ Yields calculated according to initial amount of C-allyl glycoside.

${ }^{\mathrm{b}}$ Yields calculated according to initial amount of allylgenistein.

${ }^{\mathrm{c}} \mathrm{E} / \mathrm{Z}$ molar ratio was calculated from ${ }^{1} \mathrm{H}$ NMR spectra.

${ }^{\mathrm{d}} \mathrm{CM}$ reactions initiated by II generation Grubbs complex.

(Applied Biosystem/MDS SCIEX, Foster City, CA, USA). For data acquisition, Analyst software (version 1.4) was used. The optimum mass spectrometer parameters for the detection of compounds were as follows: ion spray voltage (IS) at $4000 \mathrm{~V}$, ion source gas 1 (GS1) at $40 \mathrm{psi}$, ion source gas 2 (GS2) at $60 \mathrm{psi}$, curtain gas (CUR) at $10 \mathrm{psi}$, declustering potential (DP) at $90 \mathrm{~V}$, and entrance potential (EP) $10 \mathrm{~V}$. The source temperature was set for $600^{\circ} \mathrm{C}$. As the ion source gas and collision gas high-pressure $\mathrm{N}_{2}$ was used. The mass spectrometer operated in the positive electrospray ionization (ESI) mode. Full scan mass spectra were acquired from $m / z 1$ to 700 in $1 \mathrm{~s}$ cycle time.

\section{Results and Discussion}

Terminal olefins were synthetized by well-known procedures: C-allyl glycosides (1a-1 h) (Figure 1) were obtained in reactions with trimethylallylsilane catalyzed by Lewis acid [18-20], and 1-C-methylidene-D-glucopyranoside (1i) was obtained via Ramberg-Backlund rearrangement [18, 19], 7-Oallylgenistein (2) was synthesized from tetrabutyl ammonium salt of genistein and allyl bromide [20].

3.1. Cross-Metathesis Reactions. The presented reactions (Figure 1) were promoted by first and second generation Grubbs catalysts (Table 1). When exposed to air or complexing agents, Grubbs' complexes deactivate or change their catalytic behavior [21]. Thus Schlenck type apparatus and high purity of reactants were used to conduct reactions with high yield. In our experiments we utilized suspensions of Grubbs catalysts in paraffin, since handling such prepared initiator is much easier [22]; it can be stored without inert gas protection and weighted with higher precision. All reactions were performed under $\mathrm{Ar}$ atmosphere in refluxing $\mathrm{CH}_{2} \mathrm{Cl}_{2}$. A closely related problem is the removal of the $\mathrm{Ru}$ complex and products of its decomposition from the reaction mixture $[23,24]$. The formed genistein glycoconjugates have had to be chromatographed at least twice in order to get colorless substances.

The result of a CM reaction can be described by several parameters: overall yield, which depends on initiator stability, selectivity, which can be described by the ratio of homodimers to a CM product $\left(1: n_{\mathrm{GG}} / n_{\mathrm{FG}}: n_{\mathrm{FF}} / n_{\mathrm{FG}}\right)$, and stereoselectivity, which can be described by the molar ratio of $\mathrm{E} / \mathrm{Z}$ isomers.

The observed turnover numbers were between 4 and 22, whereas, according to the known literature, usual turnover numbers are between 5 and 20 [25-30]. If turnover number is insufficient to provide complete conversion of a substrate, a common method is to increase the amount of metathesis initiator. However, this approach has a few disadvantages, such as the increase of the cost of the synthesis and the increase of the amount of impurities originating from $\mathrm{Ru}$ complex decomposition. Therefore, we decided to use no more than $6 \mathrm{~mol} \%$ of initiator according to the amount of C-allyl glycoside. 

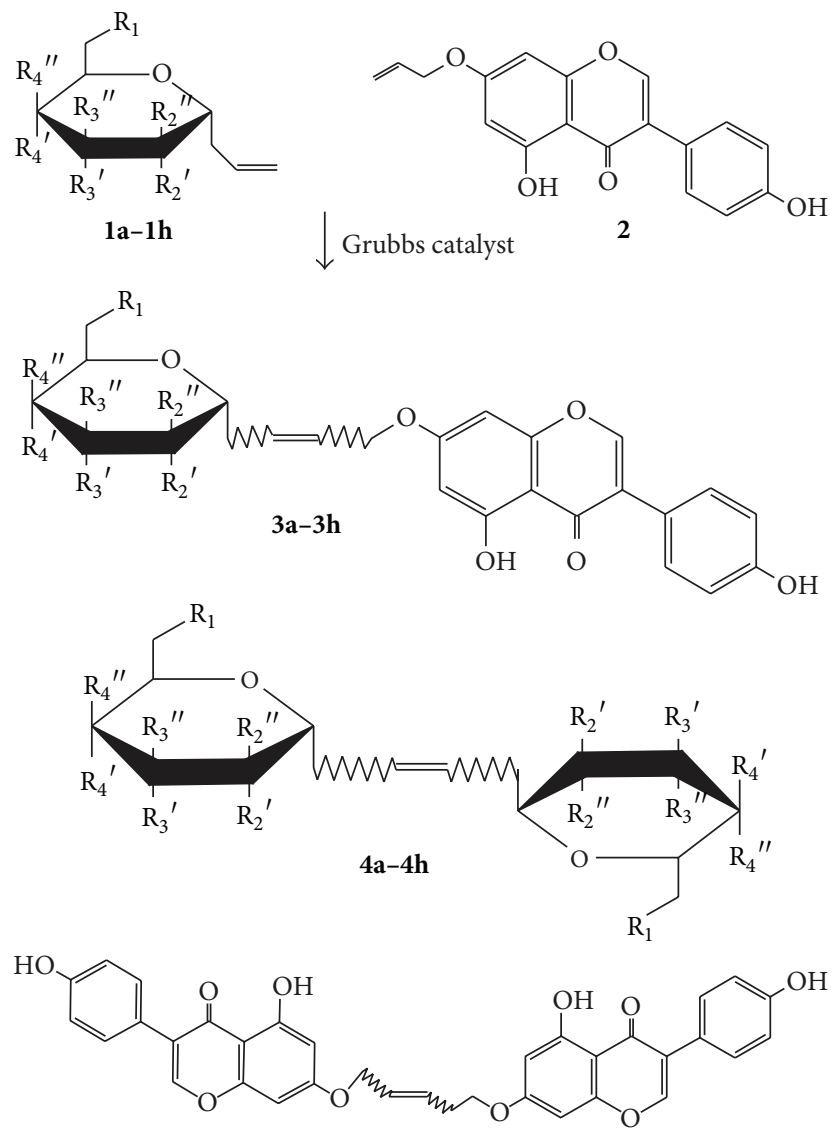

5

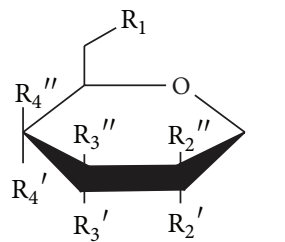

a: $\mathrm{R}_{1}=\mathrm{R}_{2}{ }^{\prime}=\mathrm{R}_{3}{ }^{\prime \prime}=\mathrm{R}_{4}{ }^{\prime}=$ OAC; $\mathrm{R}_{2}{ }^{\prime \prime}=\mathrm{R}_{3}{ }^{\prime}=\mathrm{R}_{4}{ }^{\prime \prime}=\mathrm{H}$

b: $\mathrm{R}_{1}=\mathrm{R}_{2}{ }^{\prime}=\mathrm{R}_{3}{ }^{\prime \prime}=\mathrm{R}_{4}{ }^{\prime \prime}=\mathrm{R}_{5}=\mathrm{OAc} ;=\mathrm{R}_{2}{ }^{\prime \prime}=\mathrm{R}_{3}{ }^{\prime}=\mathrm{R}_{4}{ }^{\prime}=\mathrm{H}$

c: $\mathrm{R}_{1}=\mathrm{R}_{2}{ }^{\prime \prime}=\mathrm{R}_{3}{ }^{\prime \prime}=\mathrm{R}_{4}{ }^{\prime}=\mathrm{R}_{5}=\mathrm{OAc} ;=\mathrm{R}_{2}{ }^{\prime}=\mathrm{R}_{3}{ }^{\prime}=\mathrm{R}_{4}{ }^{\prime \prime}=\mathrm{H}$

d: $\mathrm{R}_{1}=\mathrm{R}_{2}{ }^{\prime \prime}=\mathrm{R}_{3}{ }^{\prime \prime}=\mathrm{R}_{4}{ }^{\prime}=\mathrm{OBn} ; \mathrm{R}_{2}{ }^{\prime}=\mathrm{R}_{3}{ }^{\prime}=\mathrm{R}_{4}{ }^{\prime \prime}=\mathrm{H}$

e: $\mathrm{R}_{3}{ }^{\prime \prime}=\mathrm{R}_{4}{ }^{\prime}=\mathrm{Ac} ; \mathrm{R}_{2}{ }^{\prime}=\mathrm{R}_{2}{ }^{\prime \prime}=\mathrm{R}_{3}{ }^{\prime}=\mathrm{R}_{4}{ }^{\prime \prime}=\mathrm{H}$

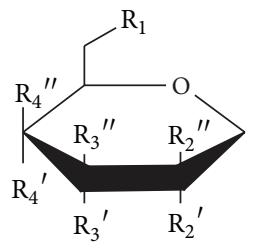

f: $\mathrm{R}_{1}=\mathrm{CH}_{3} ; \mathrm{R}_{2}{ }^{\prime}=\mathrm{OAc} ; \mathrm{R}_{2}{ }^{\prime \prime}=\mathrm{H}$

g: $\mathrm{R}_{1}=\mathrm{R}_{2}{ }^{\prime}=\mathrm{OAc} ; \mathrm{R}_{2}{ }^{\prime \prime}=\mathrm{H}$

h: $\mathrm{R}_{1}=\mathrm{R}_{2}{ }^{\prime \prime}=\mathrm{OAc} ; \mathrm{R}_{2}{ }^{\prime}=\mathrm{H}$

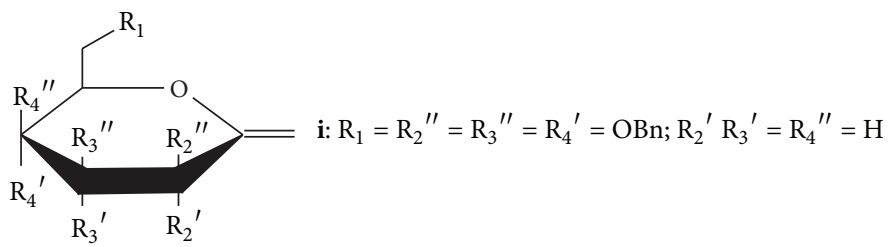

FIGURE 1: Olefin cross-metathesis reaction between C-allylglycoside (1a-1h) and 7-O-allylgenistein (2).

Another problem of CM reactions is their selectivity. In reactions described herein, six products were obtained from only two substrates (Figure 1). The selectivity of the process is decreased by the formation of homodimers (compounds 4 and 5) and can be predicted if the ability of olefins to undergo homodimerisation is known. Grubbs and coworkers divided terminal olefins into four groups, according to their reactivity in CM [31]: type I olefins undergo a rapid homodimerization, and their homodimers are consumable in the reaction; type II olefins undergo a slow homodimerization 
and their homodimers are also consumable in a much lower rate; type III olefins do not undergo homodimerisation and participate in the reaction only if an olefin of type I or II is present; and type IV olefins do not participate in the reaction at all. When two olefins of the same type are reacted with each other, formation of all possible metathesis products can be observed. However, when an olefin of high reactivity (type I) is reacted with excess of an olefin which undergoes a slow or no homodimerization (type II or III), selective CM can be achieved. Therefore we made attempts to establish the reactivity of the utilized $\mathrm{C}$-allyl glycosides and 7-Oallylgenistein. In CM reactions initiated by first generation Grubbs catalysts olefins $\mathbf{l a}$ to $\mathbf{l h}$ are type II olefins. They do undergo homodimerisation; however, their homodimers do not participate in CM (Table 1, entries: 1-8). Compound $\mathbf{l i}$ is type IV olefin. When reacted with $\mathbf{2}$, only formation of homodimer $\mathbf{5}$ could be observed. Grubbs and coworkers have reported unusually high reactivity of $\alpha, \beta$-unsaturated carbonyl compounds like acrylic acid [32]. Therefore, we have conducted an experiment with the second generation $\mathrm{Ru}$ catalyst, li and acrylic acid. Unfortunately not even traces of a CM product could be found in the reaction mixture. Most of the presented experiments are CM reactions between two olefins of second type. Such reactions are examples of nonselective $\mathrm{CM}$ reactions, and all possible products can be formed.

In contrast to ring closing metathesis (RCM) reactions $[33,34], \mathrm{CM}$ reactions usually suffer from poor stereoselectivity. Products of olefin cross-metathesis reactions were obtained as mixtures of $E / Z$ isomers. The ratio of $E / Z$ isomers was about 2 when first generation of Grubbs catalysts was used. Some improvement was achieved with second generation of Grubbs catalysts used as a metathesis initiator. The ratio of $\mathrm{E} / \mathrm{Z}$ isomers increased then to 10 and 7.3, but the yields were significantly lower (Table 1, entries 1 and 8, resp.). Whether the observed improvement was due to secondary CM $[35,36]$ is a matter of question. In our experiments we did not reach complete conversion of substrates, thus CM reactions of products should be inhibited by the presence of the more reactive substrates. On the other hand Grubbs and coworkers have shown that at low conversions, reactions initiated by second generation catalysts gave a product with low $\mathrm{E} / \mathrm{Z}$ ratio $[35,36]$.

\subsection{Preliminary Results on Biological Activity and Stabil-} ity in Culture Medium. For evaluation of antiproliferative activity of the new $\mathrm{C}$-glycosidic derivatives of genistein two compounds, $\mathbf{3 a}$ and $\mathbf{3} \mathbf{f}$, the analogs of previously tested glycoconjugates Glu-3 and Ram-3, respectively, [17] were selected. We performed a standard MTT assay in two human cancer cell lines: prostate cancer-derived DU 145 and colon cancer-derived HCT 116.

The derivatives showed the ability to inhibit the proliferation of cells in a dose-dependent manner in both cell lines, although DU 145 was less sensitive than HCT 116. The activity of the genistein derivatives, expressed as $\mathrm{IC}_{50}$ (the concentration necessary to inhibit the proliferation of cells to $50 \%$ of control) (Table 2), was higher than activity of genistein [17]. The results clearly show that the C-glycosides
TABLE 2: $\mathrm{IC}_{50}$ values of compounds $\mathbf{3} \mathbf{a}$ and $\mathbf{3} \mathbf{f}$ measured by MTT assay after $72 \mathrm{~h}$ treatment of DU 145 and HCT 116 cell lines.

\begin{tabular}{lcc}
\hline & \multicolumn{2}{c}{$\mathrm{IC}_{50}$ value $(\mu \mathrm{M})$} \\
& DU 145 & HCT 116 \\
\hline 3a & $9.50 \pm 2.21$ & $4.20 \pm 1.11$ \\
3f & $24.22 \pm 6.77$ & $8.99 \pm 2.41$ \\
Genistein* $^{*}$ & $47.29 \pm 11.78$ & $34.90 \pm 9.84$ \\
\hline
\end{tabular}

${ }^{*}$ Data from [17].

TABLE 3: Retention times and masses analyzed for the genistein Cglycosides.

\begin{tabular}{lcc}
\hline Analyte & Retention time (min) & $\mathrm{m} / z$ \\
\hline 3a & Standards \\
3f & $7.93 ; 8.25$ & 655 \\
\hline & $10.7 ; 11.01$ & 479 \\
\hline 3a & Compound incubated in cell culture medium \\
3f & $7.97 ; 8.28$ & 655 \\
\hline
\end{tabular}

of genistein described herein have enhanced antiproliferative activity in comparison to a parent compound, like their Oglycosidic counterparts.

Microscope observations showed that cells treated with 3a and $\mathbf{3 f}$ were rounded and vacuolated (Figures 2(a), 2(c), and 2(e)). They detached easily from the bottom of a dish and floated in the culture medium. The confocal analysis showed that mitochondria and lysosomes were apparently unaffected, while the structure of nuclei in many cells was altered. Significant number of cells had fragmented nuclei (Figures 2(b), 2(d), and 2(f)). This observation suggested that the tested compounds acted in a similar manner to other genistein glycoconjugates which disturbed the mitotic apparatus [17].

In the next step we evaluated the influence of Cglycoconjugates on the cell cycle. The $\mathrm{C}$-glycosidic derivatives described herein inhibited profoundly the cell cycle at G2/M phase in both cell lines tested: HCT 116 and DU 145 (Figure 3). The mode of action of the tested compounds resembled the mode of other previously described genistein derivatives substituted with modified rhamnal via a three-carbon atom linker containing O-glycosidic bond [17].

3.3. Stability of Compounds in Culture Media. In the next step we performed an analysis of stability of the tested compounds in culture media with and without cells after different duration of incubation. Growth media were collected from the culture dishes containing cultures of HCT 116 cells after 8 or $24 \mathrm{~h}$ treatment with $10 \mu \mathrm{M}$ 3a and $3 \mathbf{f}$. In control samples the tested compounds were incubated only with cell culture medium RPMI, containing $10 \%$ fetal bovine serum and $0.1 \%$ gentamycin sulfate. The analytes were analyzed by LC-MS/MS.

Based on analyses of mass spectra (Table 3 ) we found that the structure of $\mathbf{3} \mathbf{a}$ is not altered after the incubation in 


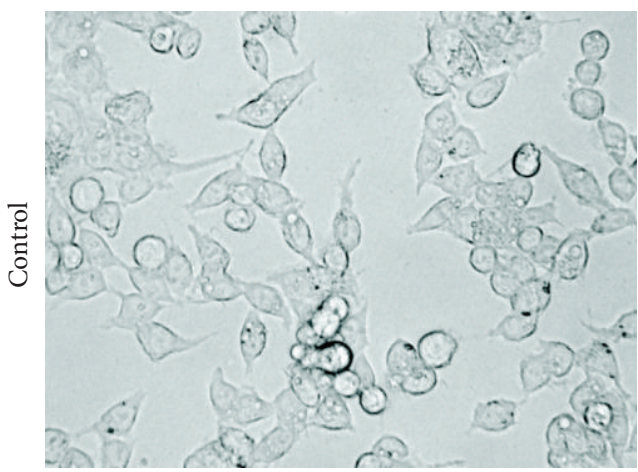

(a)

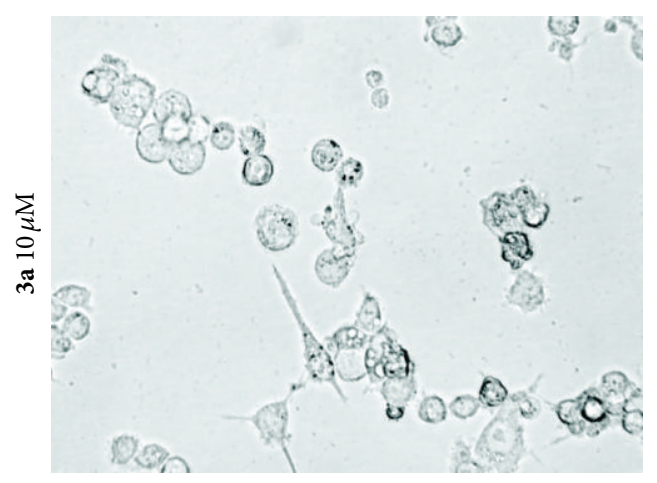

(c)

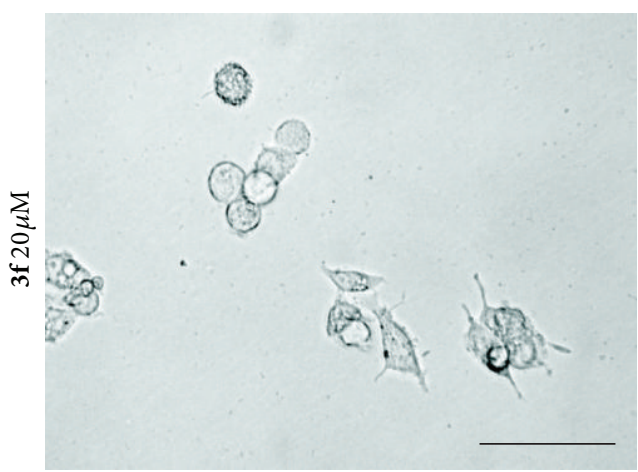

(e)

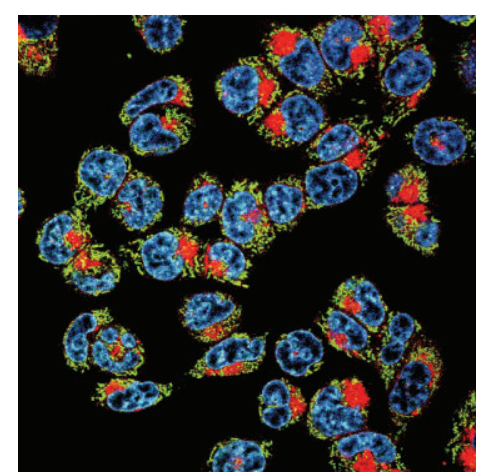

(b)

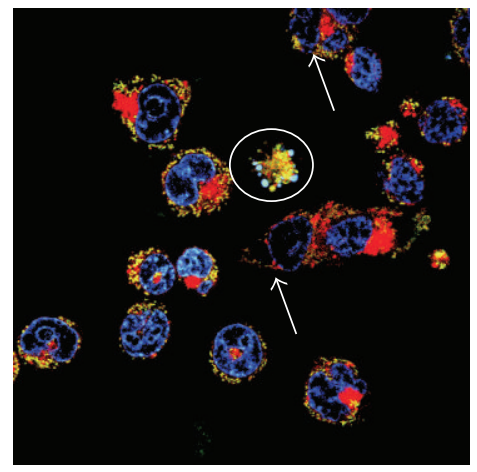

(d)

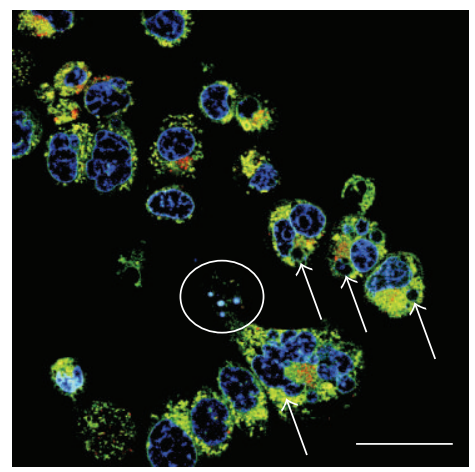

(f)

FIGURE 2: Morphology of HCT 116 cells treated with C-glycoconjugates of genistein for $24 \mathrm{~h}$. (a), (b) Non-treated control; (c), (d) $5 \mu \mathrm{M} 3 \mathbf{a}$; (e), (f) $20 \mu \mathrm{M} 3 \mathrm{f}$. ((a), (c), and (e) DIC contrast; (b), (d), and (f) confocal images of cells stained with Hoechst 33342, Lysotracker red, and Mitotracker green). Circles in (d) and (f) mark apoptotic cells, arrows indicate cell with fragmented nuclei or with large micronuclei. Scale bar: $40 \mu \mathrm{m}$ in left panel ((a), (c), and (e)); $20 \mu \mathrm{m}$ in the right panel ((b), (d), and (f)).

cell culture medium (in both cell-free medium and medium collected from the cell cultures). In contrast, $\mathbf{3} \mathbf{f}$ was less stable in culture medium (both in control medium and the medium aspirated from cell cultures). On the basis of the difference of the mass of the compound before and after incubation in culture media, we propose that the protecting acetyl group had detached. Besides of this minor structural change, the basic skeleton of a molecule was not altered during the incubation of this compound in culture medium.

\section{Conclusions}

It has been shown that application of olefin CM reaction initiated by first and second generation of Grubbs catalysts is an useful approach for the synthesis of new genistein glycoconjugates. In vitro experiments in cancer cells have shown that C-glycosidic derivatives of genistein exhibited similar mechanism of action to their O-glycosidic counterparts. These compounds were stable in a culture medium. 

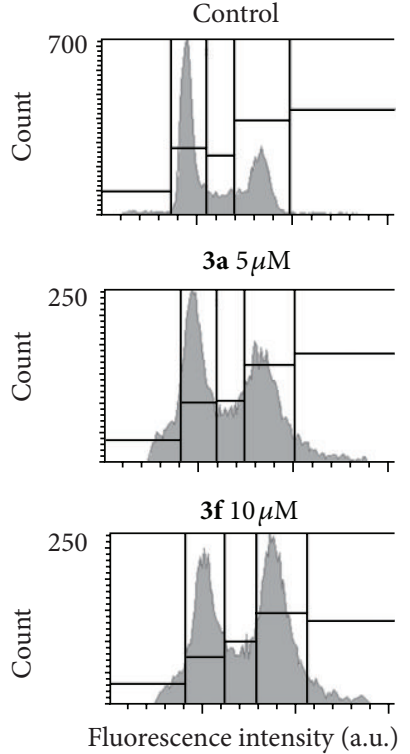

Fluorescence intensity (a.u.)

(a)

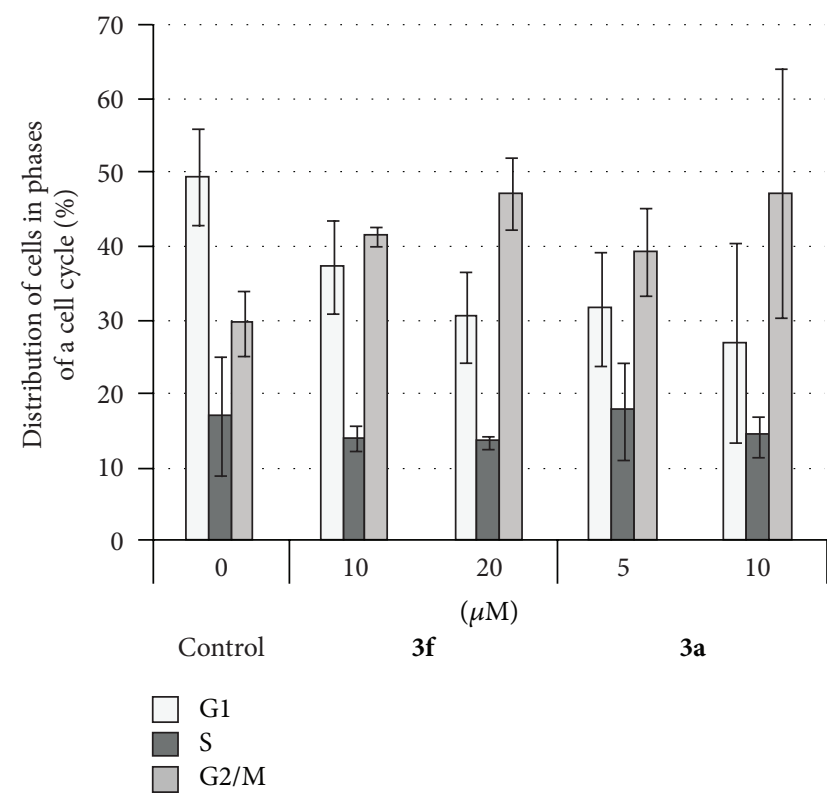

(c)

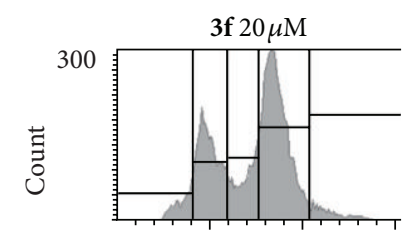

Fluorescence intensity (a.u.)
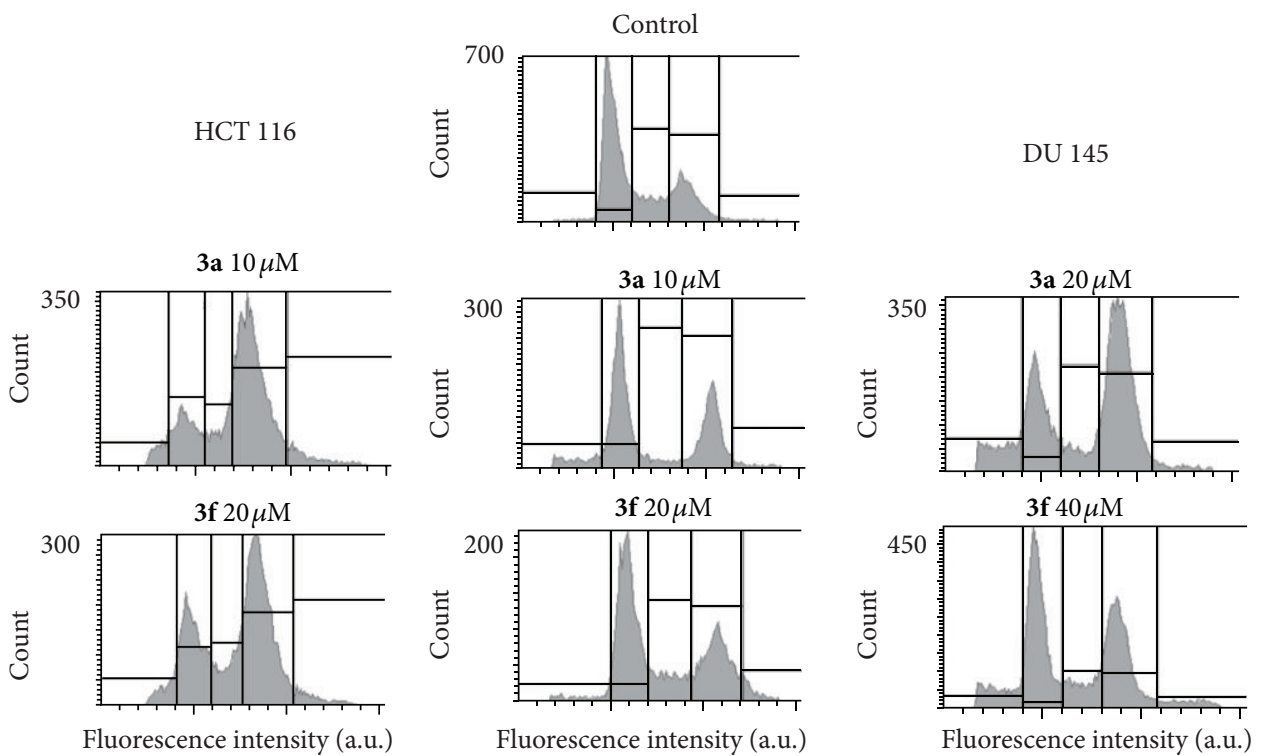

Fluorescence intensity (a.u.)

(b)

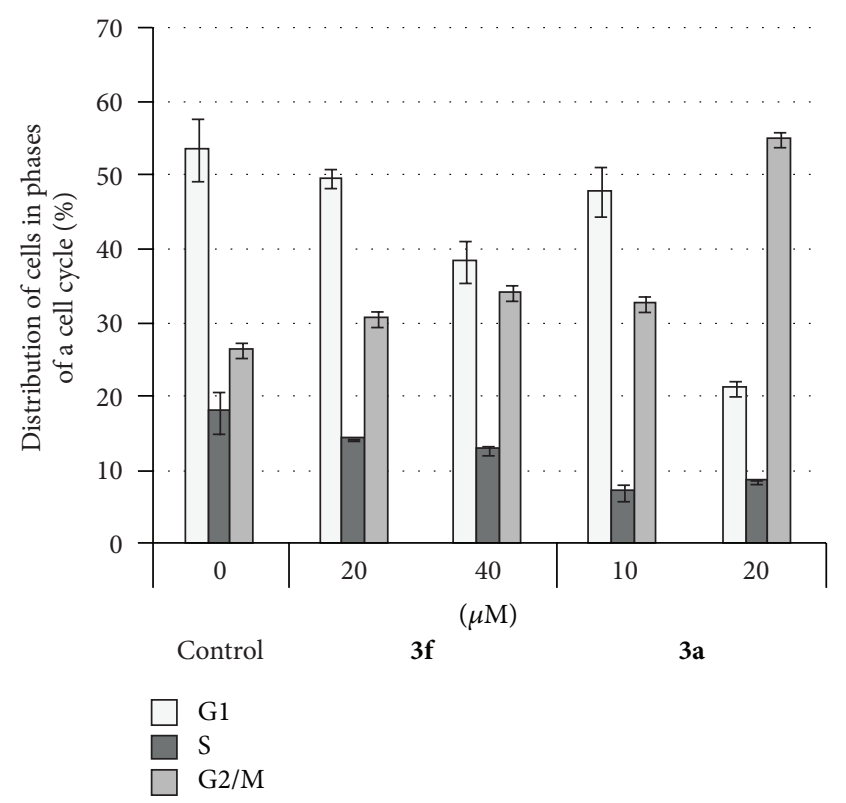

(d)

Figure 3: Cell cycle phases distribution in HCT 116 cells ((a), (c)) and DU 145 ((b), (d)) treated for 24h with 3a and 3f used at the concentrations corresponding to their cytotoxic $\mathrm{IC}_{50}$ and $2 \mathrm{xIC}_{50}$. (a), (b) Representative histograms: (c), (d) mean values and standard deviations of percentage of cells in different phases of a cell cycle. a.u.; arbitrary units.

\section{Acknowledgments}

Research studies were part-financed by the European Union within the European Regional Development Fund (POIG.01.01.02-14-102/09, POIG.02.01.00-00-166/08). Ph.D. scholarship of KP was provided by the European Union Structural Funds in Poland (UDA-POKL.04.01.01-00-114/0901).

\section{References}

[1] K. Szkudelska and L. Nogowski, "Genistein-A dietary compound inducing hormonal and metabolic changes," Journal of Steroid Biochemistry and Molecular Biology, vol. 105, no. 1-5, pp. 37-45, 2007.

[2] S. Banerjee, Y. Li, Z. Wang, and F. H. Sarkar, "Multi-targeted therapy of cancer by genistein," Cancer Letters, vol. 269, no. 2, pp. 226-242, 2008. 
[3] V. B. Gencel, M. M. Benjamin, S. N. Bahou, and R. A. Khalil, "Vascular effects of phytoestrogens and alternative menopausal hormone therapy in cardiovascular disease," Mini Reviews in Medicinal Chemistry, vol. 12, no. 2, pp. 149-174, 2012.

[4] J. Lappe, I. Kunz, I. Bendik et al., "Effect of a combination of genistein, polyunsaturated fatty acids and vitamins D3 and K1 on bone mineral density in postmenopausal women: a randomized, placebo-controlled, double-blind pilot study," European Journal of Nutrition, vol. 52, no. 1, pp. 203-215, 2013.

[5] A. Cassidy, "Potential risks and benefits of phytoestrogen-rich diets," International Journal for Vitamin and Nutrition Research, vol. 73, no. 2, pp. 120-126, 2003.

[6] H. Marini, L. Minutoli, F. Polito et al., "Effects of the phytoestrogen genistein on bone metabolism in osteopenic postmenopausal women: a randomized trial," Annals of Internal Medicine, vol. 146, no. 12, pp. 839-847, 2007.

[7] A. Rusin, Z. Krawczyk, G. Grynkiewicz, A. Gogler, J. ZawiszaPuchałka, and W. Szeja, "Synthetic derivatives of genistein, their properties and possible applications," Acta Biochimica Polonica, vol. 57, no. 1, pp. 23-34, 2010.

[8] A. H. Wu, R. G. Ziegler, P. L. Horn-Ross et al., "Tofu and risk of breast cancer in Asian-Americans," Cancer Epidemiology Biomarkers and Prevention, vol. 5, no. 11, pp. 901-906, 1996.

[9] P. This, A. De la Rochefordière, K. Clough, A. Fourquet, and H. Magdelenat, "Phytoestrogens after breast cancer," EndocrineRelated Cancer, vol. 8, no. 2, pp. 129-134, 2001.

[10] T. Usui, "Pharmaceutical prospects of phytoestrogens," Endocrine Journal, vol. 53, no. 1, pp. 7-20, 2006.

[11] J. B. Harborne and C. A. Williams, "Advances in flavonoid research since 1992," Phytochemistry, vol. 55, no. 6, pp. 481-504, 2000.

[12] F. Kohen, B. Gayer, T. Kulik et al., "Synthesis and evaluation of the antiproliferative activities of derivatives of carboxyalkyl isoflavones linked to N-t-Boc-hexylenediamine," Journal of Medicinal Chemistry, vol. 50, no. 25, pp. 6405-6410, 2007.

[13] H. Q. Li, H. M. Ge, Y. X. Chen et al., "Synthesis and cytotoxic evaluation of a series of genistein derivatives," Chemistry and Biodiversity, vol. 3, no. 4, pp. 463-472, 2006.

[14] L. N. Zhang, Z. P. Xiao, H. Ding et al., "Synthesis and cytotoxic evaluation of novel 7-O-modified genistein derivatives," Chemistry and Biodiversity, vol. 4, no. 2, pp. 248-255, 2007.

[15] K. Polkowski, J. Popiołkiewicz, P. Krzeczyński et al., "Cytostatic and cytotoxic activity of synthetic genistein glycosides against human cancer cell lines," Cancer Letters, vol. 203, no. 1, pp. 5969, 2004.

[16] J. Popiołkiewicz, K. Polkowski, J. S. Skierski, and A. P. Mazurek, "In vitro toxicity evaluation in the development of new anticancer drugs-Genistein glycosides," Cancer Letters, vol. 229, no. 1, pp. 67-75, 2005.

[17] A. Rusin, J. Zawisza-Puchałka, K. Kujawa et al., "Synthetic conjugates of genistein affecting proliferation and mitosis of cancer cells," Bioorganic and Medicinal Chemistry, vol. 19, no. 1, pp. 295305, 2011.

[18] D. E. Paterson, F. K. Griffin, M. L. Alcaraz, and R. J. K. Taylor, "A Ramberg-Bäcklund approach to the synthesis of C-glycosides, C-linked disaccharides, and C-glycosyl amino acids," European Journal of Organic Chemistry, no. 7, pp. 1323-1336, 2002.

[19] F. K. Griffin, D. E. Paterson, P. V. Murphy, and R. J. K. Taylor, "A new route to exo-glycals using the Ramberg-Bäcklund rearrangement," European Journal of Organic Chemistry, no. 7, pp. 1305-1322, 2002.
[20] G. Grynkiewicz, O. Zegrocka-Stendel, W. Pucko et al., "X-ray and C-13 CP MAS investigations of structure of two genistein derivatives," Journal of Molecular Structure, vol. 694, no. 1-3, pp. 121-129, 2004.

[21] M. B. Dinger and J. C. Mol, "Degradation of the first-generation Grubbs metathesis catalyst with primary alcohols, water, and oxygen. Formation and catalytic activity of ruthenium(II) monocarbonyl species," Organometallics, vol. 22, no. 5, pp. 1089-1095, 2003.

[22] D. F. Taber and K. J. Frankowski, "Grubbs' catalyst in paraffin: an air-stable preparation for alkene metathesis," Journal of Organic Chemistry, vol. 68, no. 15, pp. 6047-6048, 2003.

[23] M. Westhus, E. Gonthier, D. Brohm, and R. Breinbauer, "An efficient and inexpensive scavenger resin for Grubbs' catalyst," Tetrahedron Letters, vol. 45, no. 15, pp. 3141-3142, 2004.

[24] H. D. Maynard and R. H. Grubbs, "Purification technique for the removal of ruthenium from olefin metathesis reaction products," Tetrahedron Letters, vol. 40, no. 22, pp. 4137-4140, 1999.

[25] R. Roy, R. Dominique, and S. K. Das, "Extended alkenyl glycosides by ruthenium-catalyzed cross-metathesis reaction and application toward novel C-linked pseudodisaccharides," Journal of Organic Chemistry, vol. 64, no. 15, pp. 5408-5412, 1999.

[26] Y. J. Hu and R. Roy, "Cross-metathesis of N-alkenyl peptoids with O- or C-allyl glycosides," Tetrahedron Letters, vol. 40, no. 17, pp. 3305-3308, 1999.

[27] A. Kirschning and G. W. Chen, "Syntheses of spacer-linked neodisaccharides derived from L-Daunosamine," Tetrahedron Letters, vol. 40, no. 25, pp. 4665-4668, 1999.

[28] D. J. O'Leary, H. E. Blackwell, R. A. Washenfelder, and R. H. Grubbs, "A new method for cross-metathesis of terminal olefins," Tetrahedron Letters, vol. 39, no. 41, pp. 7427-7430, 1998.

[29] H. E. Blackwell, D. J. O'Leary, A. K. Chatterjee, R. A. Washenfelder, D. A. Bussmann, and R. H. Grubbs, "New approaches to olefin cross-metathesis," Journal of the American Chemical Society, vol. 122, no. 1, pp. 58-71, 2000.

[30] D. Forget-Champagne, M. Mondon, N. Fonteneau, and J. P. Gesson, "Selective cross-metathesis of 2-allylphenols with styrenes," Tetrahedron Letters, vol. 42, no. 41, pp. 7229-7231, 2001.

[31] A. K. Chatterjee, T. L. Choi, D. P. Sanders, and R. H. Grubbs, "A general model for selectivity in olefin cross metathesis," Journal of the American Chemical Society, vol. 125, no. 37, pp. 1136011370, 2003.

[32] T. L. Choi, Choon Woo Lee, A. K. Chatterjee, and R. H. Grubbs, "Olefin metathesis involving ruthenium enoic carbene complexes," Journal of the American Chemical Society, vol. 123, no. 42, pp. 10417-10418, 2001.

[33] T. Velasco-Torrijos and P. V. Murphy, "Metathesis of structurally preorganized bivalent carbohydrates. Synthesis of macrocyclic and oligomeric scaffolds," Organic Letters, vol. 6, no. 22, pp. 3961-3964, 2004.

[34] M. H. D. Postema and J. L. Piper, "Cross-metathesis and ringclosing metathesis of olefinic monosaccharides," Tetrahedron Letters, vol. 43, no. 39, pp. 7095-7099, 2002.

[35] T. Ritter, A. Hejl, A. G. Wenzel, T. W. Funk, and R. H. Grubbs, "A standard system of characterization for olefin metathesis catalysts," Organometallics, vol. 25, no. 24, pp. 5740-5745, 2006.

[36] D. R. Anderson, T. Ung, G. Mkrtumyan, G. Bertrand, R. H. Grubbs, and Y. Schrodi, "Kinetic selectivity of olefin metathesis catalysts bearing cyclic (alkyl)(amino)carbenes," Organometallics, vol. 27, no. 4, pp. 563-566, 2008. 

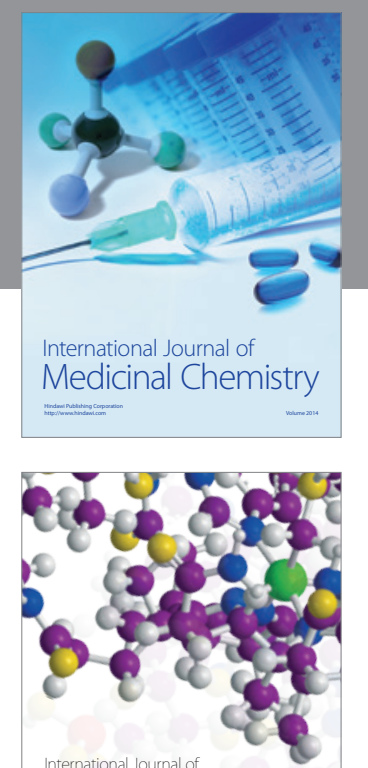

\section{Carbohydrate} Chemistry

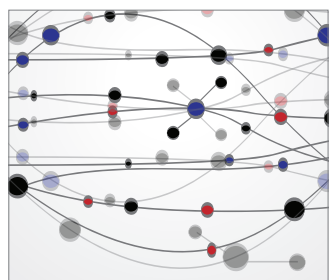

The Scientific World Journal
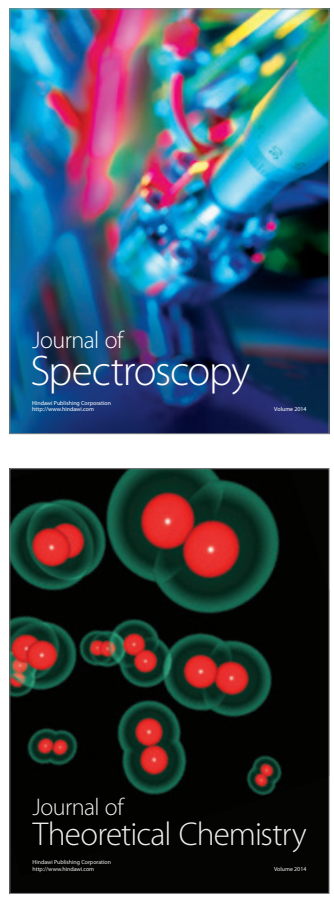
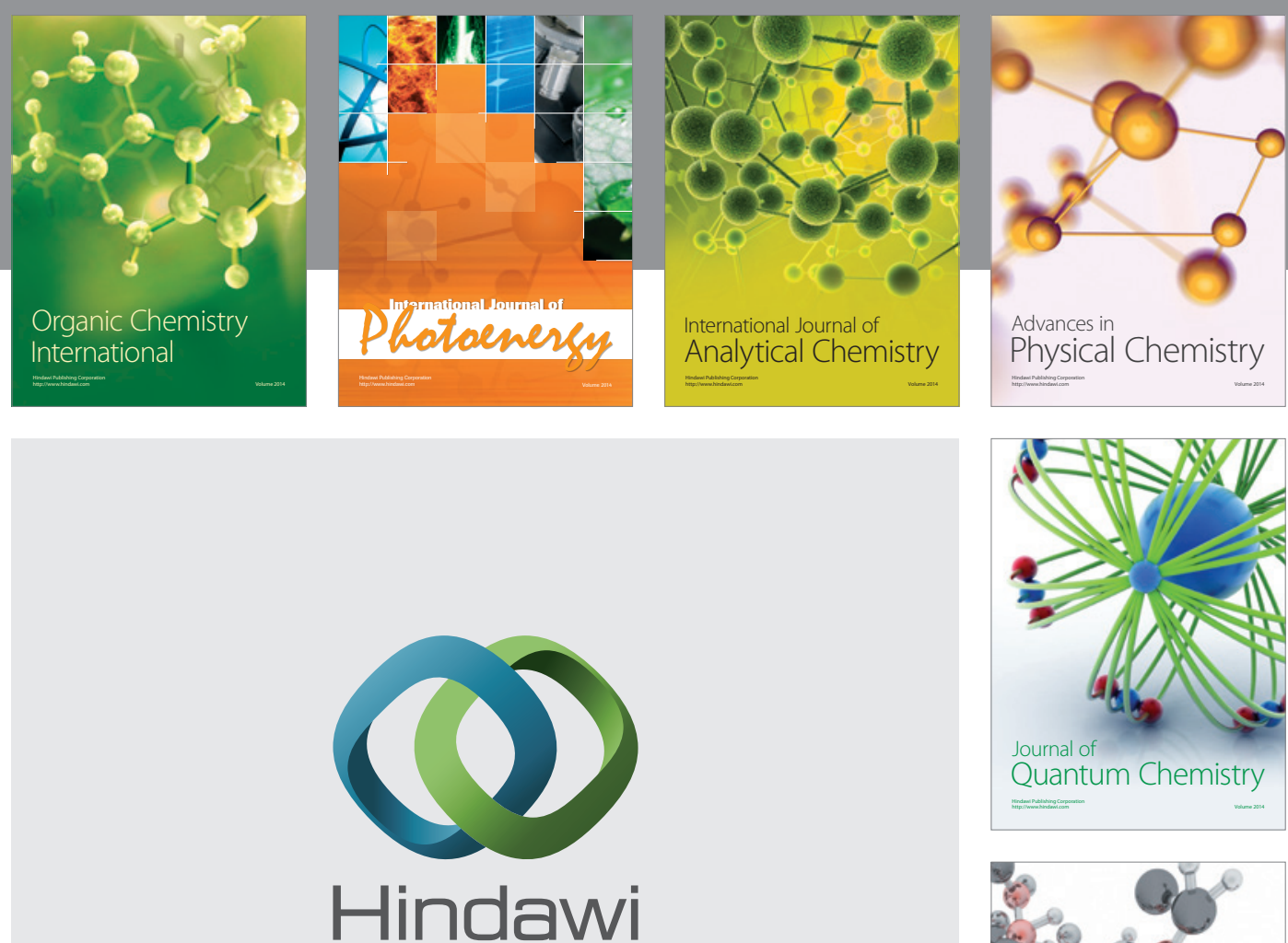

Submit your manuscripts at

http://www.hindawi.com

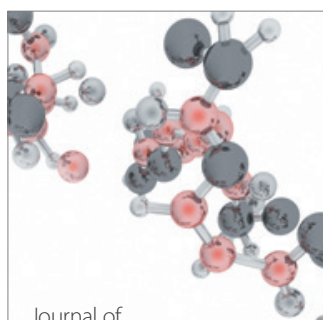

Analytical Methods

in Chemistry

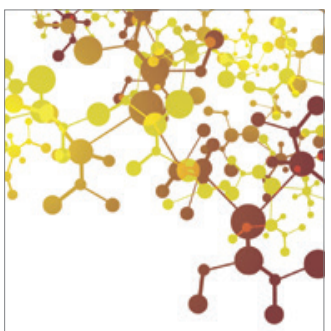

Journal of

Applied Chemistry

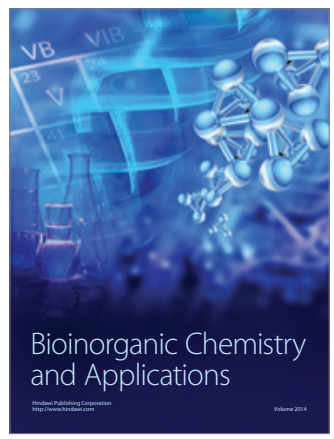

Inorganic Chemistry
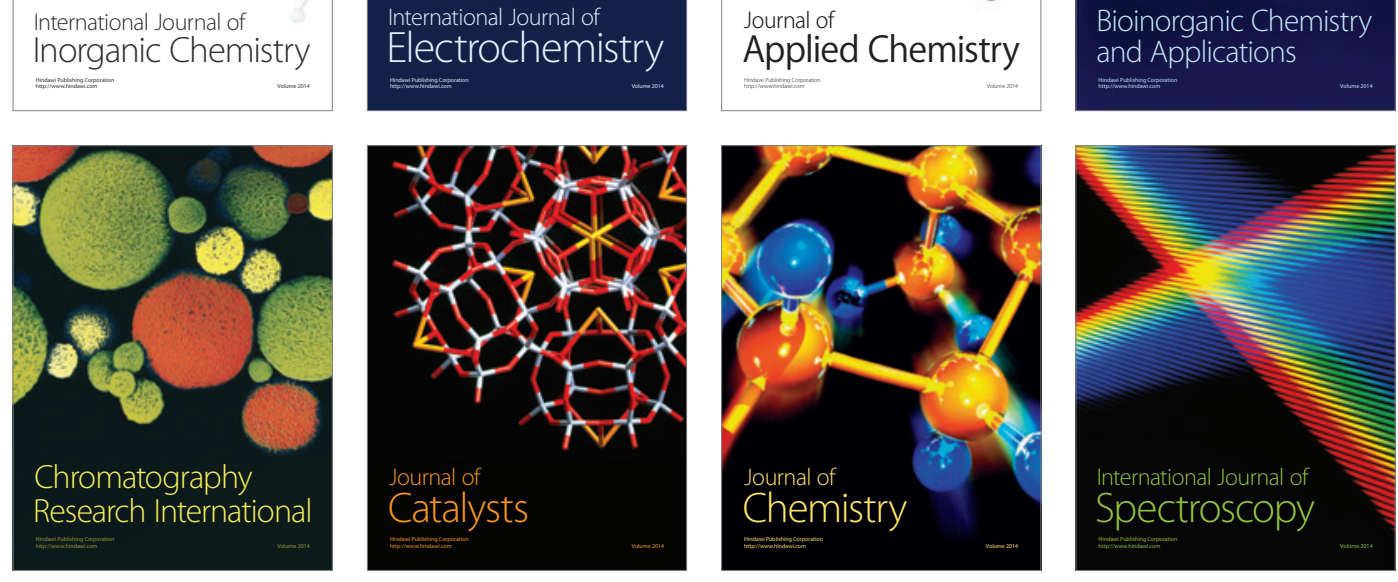